\title{
Fourier-integral-operator product representation of solutions to first-order symmetrizable hyperbolic systems
}

\author{
Jérôme Le Rousseau ${ }^{* i t}$ \\ Universités d'Aix-Marseille, Université de Provence, France \\ Laboratoire d'Analyse Topologie Probabilités, CNRS UMR 6632
}

January 29, 2009

\begin{abstract}
We consider the first-order Cauchy problem

$$
\begin{aligned}
\partial_{z} u+a\left(z, x, D_{x}\right) u & =0, \quad 0<z \leq Z, \\
\left.u\right|_{z=0} & =u_{0},
\end{aligned}
$$

with $Z>0$ and $a\left(z, x, D_{x}\right)$ a $k \times k$ matrix of pseudodifferential operators of order one, whose principal part $a_{1}$ is assumed symmetrizable: there exists $L(z, x, \xi)$ of order 0 , invertible, such that

$$
a_{1}(z, x, \xi)=L(z, x, \xi)\left(-i \beta_{1}(z, x, \xi)+\gamma_{1}(z, x, \xi)\right)(L(z, x, \xi))^{-1},
$$

where $\beta_{1}$ and $\gamma_{1}$ are hermitian symmetric and $\gamma_{1} \geq 0$. An approximation Ansatz for the operator solution, $U\left(z^{\prime}, z\right)$, is constructed as the composition of global Fourier integral operators with complex matrix phases. In the symmetric case, an estimate of the Sobolev operator norm in $L\left(\left(H^{(s)}\left(\mathbf{R}^{n}\right)\right)^{k},\left(H^{(s)}\left(\mathbf{R}^{n}\right)\right)^{k}\right)$ of these operators is provided, which yields a convergence result for the Ansatz to $U\left(z^{\prime}, z\right)$ in some Sobolev space as the number of operators in the composition goes to $\infty$, in both the symmetric and symmetrizable cases. We thus obtain a representation of the solution operator $U\left(z^{\prime}, z\right)$ as an infinite product of Fourier integral operators with matrix phases.
\end{abstract}

Keywords: Hyperbolic system; Symmetrizable system; Pseudodifferential initial value problem; Fourier integral operator; Matrix phase function; Global Sobolev norm estimate; Multi-product.

AMS 2000 subject classification: 35L40; 35L80; 35S10; 35S30.

*New adress: Université d'Orléans, Laboratoire Mathématiques et Applications, Physique Mathématique d'Orléans, CNRS UMR 6628, Fédération Denis Poisson, FR CNRS 2964, B.P. 6759, 45067 Orléans cedex 2, France.

†E-mail address: jlr@univ-orleans.fr

\$This article was written when the author was on a research leave at Laboratoire POems, INRIA / ENSTA, CNRS UMR 2706. 


\section{Contents}

Introduction

1 Some results on Fourier integral operators in the scalar case 6

1.1 A class of Fourier integral operators with complex phase . . . . . . . . . . . . 6

1.2 Action on $\mathscr{P}\left(\mathbf{R}^{n}\right)$ and $\mathscr{P}^{\prime}\left(\mathbf{R}^{n}\right) \ldots \ldots \ldots \ldots \ldots$

1.3 Some calculus results . . . . . . . . . . . . . . . . . . . 8

1.4 Sobolev space regularity $\ldots \ldots \ldots \ldots \ldots \ldots$

2 The thin-slab propagator for a symmetric system 11

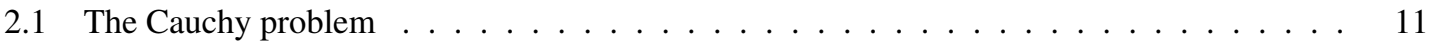

2.2 The thin-slab propagator $\ldots \ldots \ldots \ldots \ldots \ldots$

2.3 A class of symbols . . . . . . . . . . . . . . . . . . . . . . . 13

2.4 Sobolev space regularity estimate for the thin-slab propagator . . . . . . . . . . . . . . 17

3 Convergence properties of the Ansatz $\mathcal{W}_{\mathfrak{P}, z}$ in the symmetric case 22

3.1 A composition formula in the case of matrix phases . . . . . . . . . . . . 23

3.2 Error estimation, convergence $\ldots \ldots \ldots \ldots \ldots \ldots \ldots$

4 Symmetrizable systems $r$

4.1 Some operator properties . . . . . . . . . . . . . . . . . . . 26

4.2 Composition of two thin-slab propagators . . . . . . . . . . . . . . . . 27

4.3 Stability of the Ansatz $\mathcal{W}_{\mathfrak{P}, z}$ and conclusion . . . . . . . . . . . . . . 28

A Some technical proofs $\quad 32$

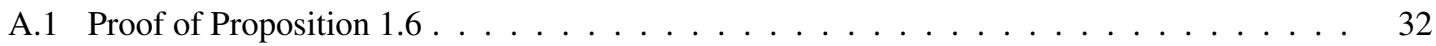

A.2 Proof of Proposition $1.10 \ldots \ldots \ldots \ldots \ldots \ldots$

A.3 Proof of Lemma $1.13 \ldots \ldots \ldots \ldots \ldots \ldots$

A.4 Proof of Theorem 2.20: sharp Gårding inequality ～. . . . . . . . . . . . . . . . . . 37

A.5 Proof of Theorem $3.4 \ldots \ldots \ldots \ldots \ldots \ldots$

A.6 Proof of Proposition 3.6 . . . . . . . . . . . . . . . . . . . . 43

A.7 Proofs of Propositions 4.4 and $4.5 \ldots \ldots \ldots \ldots \ldots$

A.8 Proofs of Propositions 4.6, 4.7 and $4.8 \ldots \ldots \ldots \ldots$. . . . . . . . . . . 47

A.9 Proofs of Lemmata 4.11 and $4.12 \ldots \ldots \ldots \ldots$. . . . . . . . . . . . . . 50

$\begin{array}{lr}\text { References } & 53\end{array}$ 


\section{Introduction and notation}

Let $k, n \in \mathbf{N}^{*}$. We consider the Cauchy problem

$$
\begin{aligned}
\partial_{z} u+a\left(z, x, D_{x}\right) u & =0, \quad 0<z \leq Z, \\
\left.u\right|_{z=0} & =u_{0},
\end{aligned}
$$

with $Z>0, u(z, x) \in \mathbf{C}^{k}$, and $a(z, x, \xi)$ a $k \times k$ matrix with entries continuous with respect to (w.r.t.) $z$ with values in $S^{1}\left(\mathbf{R}^{n} \times \mathbf{R}^{n}\right)$, with the usual notation $D_{x}=\frac{1}{i} \partial_{x}$. (Symbol spaces are precisely defined at the end of this introductory section.)

We write $a(z, x, \xi)=a_{1}(z, x, \xi)+a_{0}(z, x, \xi)$, where $a_{1}$ is the principal part of $a$ and $a_{0}$ is a matrix symbol with entries in $S^{0}\left(\mathbf{R}^{n} \times \mathbf{R}^{n}\right)$. The principal part is assumed homogeneous of degree one and symmetrizable in the sense that there exists a matrix $L(z, x, \xi)$ with entries in $S^{0}\left(\mathbf{R}^{n} \times \mathbf{R}^{n}\right)$, with $z$ as a parameter, such that

$$
a_{1}(z, x, \xi)=L(z, x, \xi) \alpha_{1}(z, x, \xi)(L(z, x, \xi))^{-1},
$$

and $\alpha_{1}(z, x, \xi)=-i \beta_{1}(z, x, \xi)+\gamma_{1}(z, x, \xi)$, where $\beta_{1}$ and $\gamma_{1}$ are hermitian symmetric $k \times k$ matrices. The matrix $\gamma_{1}(z, x, \xi)$ is also assumed non-negative. For the precise statements of the assumptions we make on the symbol $a(z, x, \xi)$ refer to the subsequent sections.

In the case where, for instance, the eigenvalue multiplicities are constant, the solution operator $U\left(z^{\prime}, z\right)$ of the Cauchy problem (1)-(2) is given by Fourier integral operators (FIO). However, in practice, the amplitudes are only known up to elements of $S^{-\infty}$, i.e., the operator itself is known up to a regularizing operator. We wish to develop an alternative representation of $U\left(z^{\prime}, z\right)$. The solution operator is approximated by a multiproduct of FIOs, that are given explicitly, and we prove convergence as the number of operators in the product goes to infinity. The convergence is not up to a regularising operator: the limit we obtain is exactly $U\left(z^{\prime}, z\right)$.

When $a(z, x, \xi)$ is scalar, $k=1$, and independent of $x$ and $z$ it is natural to treat such a problem by means of Fourier transformation:

$$
u\left(z, x^{\prime}\right)=\iint e^{i\left\langle x^{\prime}-x \mid \xi\right\rangle-z a(\xi)} u_{0}(x) d \xi d x
$$

where $d \xi:=d \xi /(2 \pi)^{n}$. Some assumption needs to be made on the symbol $a(\xi)$ for this oscillatory integral to be well defined. In particular, non-negativity will be imposed on $a(\xi)$. When the symbol $a$ depends on both $x$ and $z$ we can naively expect

$$
u\left(z, x^{\prime}\right) \approx u_{1}\left(z, x^{\prime}\right):=\iint e^{i\left\langle x^{\prime}-x \mid \xi\right\rangle-z a\left(0, x^{\prime}, \xi\right)} u_{0}(x) d \xi d x,
$$

for $z$ small, and hence approximately solve the Cauchy problem (1)-(2) for $z \in\left[0, z^{(1)}\right]$ with $z^{(1)}$ small. If we want to progress in the $z$ direction we have to solve the Cauchy problem

$$
\begin{aligned}
\partial_{z} u+a\left(z, x, D_{x}\right) u & =0, \quad z^{(1)}<z \leq Z, \\
\left.u(z, .)\right|_{z=z^{(1)}} & =u_{1}\left(z^{(1)}, .\right)
\end{aligned}
$$

which we again approximately solve by

$$
u\left(z, x^{\prime}\right) \approx u_{2}\left(z, x^{\prime}\right):=\iint e^{i\left\langle x^{\prime}-x \mid \xi\right\rangle-\left(z-z^{(1)}\right) a\left(z^{(1)}, x^{\prime}, \xi\right)} u_{1}\left(z^{(1)}, x\right) d \xi d x
$$

This procedure can be iterated until we reach $z=Z$.

In the scalar case, $k=1$, upon appropriate assumptions, this procedure converges [29, 30] and yields the solution operator of the Cauchy problem (1)-(2). The convergence is obtained in Sobolev spaces. We wish to extend this type of results to the case of a symmetrizable system, which then yields a representation of the solution operator as an infinite product of FIOs of the form of (3). The extension is intricate mainly because we have to deal with matrix symbols and phases which do not commute in general and some simple algebraic operations in the scalar case become impossible. Here, we introduce classes of FIOs with matrix 
phase functions. Some care is required for them to be well defined and some assumptions will be made on the matrix symbol $a(z, x, \xi)$, which in particular generalize those made in the scalar case in [29, 30]. We shall prove some calculus results for these operators.

We define the so-called thin-slab propagator, $\mathcal{G}_{\left(z^{\prime}, z\right)}$, as the operator with (matrix) kernel

$$
G_{\left(z^{\prime}, z\right)}\left(x^{\prime}, x\right)=\int e^{i\left\langle x^{\prime}-x \mid \xi\right\rangle} e^{-\left(z^{\prime}-z\right) a_{0}\left(z, x^{\prime}, \xi\right)} e^{-\left(z^{\prime}-z\right) a_{1}\left(z, x^{\prime}, \xi\right)} d \xi
$$

Note that $e^{-\left(z^{\prime}-z\right) a_{0}\left(z, x^{\prime}, \xi\right)}$ and $e^{-\left(z^{\prime}-z\right) a_{1}\left(z, x^{\prime}, \xi\right)}$ do not commute in general. Combining all iteration steps above involves composition of such operators: let $0 \leq z^{(1)} \leq \cdots \leq z^{(k)} \leq Z$, we then have

$$
u_{k+1}(z, x)=\mathcal{G}_{\left(z, z^{(k)}\right)} \circ \mathcal{G}_{\left(z^{(k)}, z^{(k-1)}\right)} \circ \cdots \circ \mathcal{G}_{\left(z^{(1)}, 0\right)}\left(u_{0}\right)(x),
$$

if $z \geq z^{(k)}$. We then define the operator $\mathcal{W}_{\mathfrak{P}, z}$ for a subdivision $\mathfrak{P}$ of the interval $[0, Z], \mathfrak{P}=\left\{z^{(0)}, z^{(1)}, \ldots, z^{(N)}\right\}$, with $0=z^{(0)}<z^{(1)}<\cdots<z^{(N)}=Z$,

$$
\mathcal{W}_{\mathfrak{P}, z}:= \begin{cases}\mathcal{G}_{(z, 0)} & \text { if } 0 \leq z \leq z^{(1)} \\ \mathcal{G}_{\left(z, z^{(k)}\right)} \prod_{i=k}^{1} \mathcal{G}_{\left(z^{(i)}, z^{(i-1)}\right)} & \text { if } z^{(k)} \leq z \leq z^{(k+1)} .\end{cases}
$$

According to the procedure described above, $\mathcal{W}_{\mathfrak{P}, z}$ yields an approximation Ansatz for the solution operator of the Cauchy problem (1)-(2) with step size $\Delta_{\mathfrak{P}}=\sup _{i=1, \ldots, N}\left(z^{(i)}-z^{(i-1)}\right)$.

Note that a similar procedure can be used to show the existence of an evolution system by approximating it by composition of semigroup solutions of the Cauchy problem with $z$ 'frozen' in $a\left(z, x, D_{x}\right)[18,34]$. It should be noticed that $\mathcal{W}_{\mathfrak{P}, z}$ is however not the solution operator of problem (1)-(2) even in the case where the symbol $a$ only depends on the transverse variable, $x$. For instance in the scalar case, $k=1$, while singularities propagate along the bicharacteristics associated with $-\operatorname{Im}\left(a_{1}\right)$, we however observe that, with the form of the phase function, the operator $\mathcal{G}_{\left(z^{\prime}, z\right)}$ propagates singularities along straight lines. See [32] for further details. Furthermore, by composing the operators $\mathcal{G}_{\left(z^{\prime \prime}, z^{\prime}\right)}$ and $\mathcal{G}_{\left(z^{\prime}, z\right)}$, one convinces oneself that

$$
\mathcal{G}_{\left(z^{\prime \prime}, z\right)} \neq \mathcal{G}_{\left(z^{\prime \prime}, z^{\prime}\right)} \circ \mathcal{G}_{\left(z^{\prime}, z\right)}
$$

in general if $z^{\prime \prime} \geq z^{\prime} \geq z \in[0, Z]$ (use again that singularities propagate along straight lines). The family of operators $\left(\mathcal{G}_{\left(z^{\prime}, z\right)}\right)_{\left(z^{\prime}, z\right) \in[0, Z]^{2}}$ is thus neither a semigroup nor an evolution system.

Under Hölder regularity of order $\alpha$ of $a(z, x, \xi)$ w.r.t. $z$, and Lipschitz regularity of $L(z, x, \xi)$ w.r.t. $z$ in the symmetrizable case, we shall prove convergence of the Ansatz $\mathcal{W}_{\mathfrak{P}, z}$ to the solution operator $U\left(z^{\prime}, z\right)$ of the Cauchy problem (1)-(2):

$$
\left\|\mathcal{W}_{\mathfrak{P}, z}-U(z, 0)\right\|_{\left(\left(H^{(s+1)}\right)^{k},\left(H^{(s+r)}\right)^{k}\right)} \leq \Delta_{\mathfrak{P}}^{(1-r) \alpha},
$$

for $0 \leq r<1$ (Theorem 3.7 in the symmetric case, $L(z, x, \xi)=I_{k}$, and Theorem 4.15 in the symmetrizable case). We thus obtain a representation of the solution operator $U\left(z^{\prime}, z\right)$ as an infinite product of FIOs with matrix phases. Such a result is achieved by first proving a precise estimate of the Sobolev operator norm of the thin-slab propagator $\mathcal{G}_{\left(z^{\prime}, z\right)}$ in the symmetric case, $L(z, x, \xi)=I_{k}$ : for all $s \in \mathbf{R}$, there exists $C \geq 0$ such that

$$
\left\|\mathcal{G}_{\left(z^{\prime}, z\right)}\right\|_{\left(\left(H^{(s)}\right)^{k},\left(H^{(s)}\right)^{k}\right)} \leq 1+C \Delta,
$$

for all $0 \leq z \leq z^{\prime} \leq Z$ with $\Delta=z^{\prime}-z$ sufficiently small (Theorem 2.22). To prove (5), we assume that the symbols $\beta_{1}$ and $\gamma_{1}$ commute and are diagonalizable with a diagonalizing symbol that is smooth w.r.t. $x$ and $\xi$ and bounded w.r.t. $z$, i.e. the symbol $a(z, x, \xi)$ is assumed to be "geometrically regular". Note that this assumption does not exclude the crossing of smooth eigenvalues. Estimate (5) yields the stability of the multi-composition of FIOs. For symmetrizable systems we assume that the symmetrizing symbol $L(z, x, \xi)$ is smooth w.r.t. $x$ and $\xi$ and Lipschitz continuous w.r.t. $z$. In this case, solutions of (1)-(2) exist and are unique and we prove the convergence of the proposed Ansatz $\mathcal{W}_{\mathfrak{P}, z}$ to $U(z, 0)$. An estimation of the form of (5) is however not obtained in the case of a symmetrizable system. The treatment of this case relies on a careful analysis of the product $\mathcal{G}_{\left(z^{\prime \prime}, z^{\prime}\right)} \circ \mathcal{G}_{\left(z^{\prime}, z\right)}$ that allows to fold back onto the symmetric case, yet creating 
perturbative terms that accumulate as the number of operators in the multi-composition increases. These additional perturbative terms can be managed and the stability of the multi-composition is obtained.

Multi-composition of FIOs to approximate solutions of Cauchy problems were first proposed in the scalar case in [25] and [24]. In these articles, the exact solution operator of a first-order hyperbolic equation is approximated with a different Ansatz. The approximation is made up to a regularizing operator. The technique is based on the computation and the estimation of the phase functions and amplitudes of the FIO resulting from these multi-products, a result known as the Kumano-go-Taniguchi Theorem. It is based on the earlier work of H. Kumano-go in [22]. This approach is synthesized in Chapter 10 of [23].

The case of systems with constant multiplicities in non-diagonal form in also treated in [23, Section 10.4]. However, the system is diagonalized by the application of elliptic pseudodifferential operators and the solution is only recovered through the use of a parametrix which yields the solution operator up to a regularizing operator. In the present article, we aim to obtain an exact representation of the solution operator. Hence we do not rely on such a diagonalization procedure of the system.

The multi-product technique was further applied to Schrödinger equations with specific symbols [20, 26]. In these latter works, the multi-product in also interpreted as an iterated integral of Feynman's type and convergence is studied in a weak sense. The time slicing approximation, closely related to our approach, allows to give a rigorous mathematical meaning to Feynman path integrals [27, 11]. In [20] a convergence result in $L^{2}$ is proven. This is the type of results sought here for first-order hyperbolic systems. We however do not use the apparatus of multi-phases and rather focus on estimating the Sobolev regularity of each term in the multi-product of FIOs in the proposed Ansatz $\mathcal{W}_{\mathfrak{F}, z}$. While the resulting product is an FIO, we do not compute its phase and amplitude. The Sobolev regularity allows us to use an a priori energy estimate for the Cauchy problem (1)-(2) to prove convergence of the approximating Ansatz to the solution operator.

In [36] (see also [29, Appendix A]), a (microlocal) decoupling of the up-going and down-going wavefields of the solution to the acoustic wave equation is presented. The resulting one-way wave equations are of the form of (1)-(2) in the scalar case. In practice, the proposed Ansatz $\mathcal{W}_{\mathfrak{P}, z}$ can then be used in geophysical problems for the purpose of imaging the Earth's interior [8,7]. As similar decoupling procedure can be applied to the elasticity system and we obtain two one-way systems of the form of (1)-(2) [6]. One motivation for this article is to use the proposed approximation Ansatz as a tool to compute approximations of the exact solutions to the one-way systems of elasticity. Such computations in application to geophysical problems have been used in [31]. With applications such as imaging in mind, in which one aims mainly to recover the singularities in the subsurface (see for instance $[38,5]$ ), we could extend the scalar results of [32] to the case of systems, which would then yield microlocal convergence results, i.e. convergence of the wavefront set of $\mathcal{W}_{\mathfrak{P}, z}\left(u_{0}\right)$ to that of $U(z, 0)\left(u_{0}\right)$ in the sense given in [32].

The outline of the article is as follows. In Section 1, we prove some global regularity results for classes of scalar FIOs with complex phase. These results are used in the subsequent Sections. In Section 2, we introduce the thin-slab propagator in the case of a symmetric system, $L(z, x, \xi)=I_{k}$, and prove estimate (5). In Section 3, we use this estimate to prove the stability of the Ansatz $\mathcal{W}_{\mathfrak{P}, z}$ and then prove the convergence result (4). In Section 4, we address the case of a symmetrizable system and prove the convergence result (4) in this case, assuming Lipschitz regularity of $L(z, x, \xi)$ w.r.t. $z$. Some technical proofs have been placed in Appendix A to ease the reading of the article.

In this article, when the constant $C$ is used, its value may change from one line to another. If we want to keep track of the value of a constant we shall use another letter. When we write that a function is bounded w.r.t. $z$ and/or $\Delta$ we shall actually mean that $z$ is to be taken in the interval $[0, Z]$ and $\Delta$ in some interval $\left[0, \Delta_{\mathfrak{P}}\right]$ unless otherwise stipulated. We shall generally write $X, X^{\prime}, X^{\prime \prime}, X^{(1)}, \ldots, X^{(N)}$ for $\mathbf{R}^{n}$, according to variables, e.g., $x, x^{\prime}, \ldots, x^{(N)}$. We shall sometimes use the variables $y, t$ that belong to $Y$ and $T$ which will denote $\mathbf{R}^{n_{y}}, \mathbf{R}^{n_{t}}$ with possibly $n_{y} \neq n$ and $n_{t} \neq n$. For the sake of concision we introduce

$$
\int_{(\pi)}=\underbrace{\int \cdots \int}_{n \text { times }}, \quad \text { for } n \geq 3, n \in \mathbf{N} \text {. }
$$

In a standard way, we set $\langle\xi\rangle:=\sqrt{1+|\xi|^{2}}$ for $\xi \in \mathbf{R}^{p}$. Throughout the article, we use spaces of global symbols; a function $a \in \mathscr{C}^{\infty}\left(\mathbf{R}^{q} \times \mathbf{R}^{p}\right)$ is in $S_{\rho, \delta}^{m}\left(\mathbf{R}^{q} \times \mathbf{R}^{p}\right), 0<\rho \leq 1,0 \leq \delta<1$, if for all multi-indices $\alpha, \beta$ 
there exists $C_{\alpha \beta}>0$ such that

$$
\left|\partial_{x}^{\alpha} \partial_{\xi}^{\beta} a(x, \xi)\right| \leq C_{\alpha \beta}\langle\xi\rangle^{m-\rho|\beta|+\delta|\alpha|}, \quad x \in \mathbf{R}^{q}, \xi \in \mathbf{R}^{p} .
$$

The best possible constants $C_{\alpha \beta}$, i.e.,

$$
p_{\alpha \beta}(a):=\sup _{(x, \xi) \in \mathbf{R}^{q} \times \mathbf{R}^{p}}\langle\xi\rangle^{-m+\rho|\beta|-\delta|\alpha|}\left|\partial_{x}^{\alpha} \partial_{\xi}^{\beta} a(x, \xi)\right|,
$$

define seminorms for a Fréchet space structure on $S_{\rho, \delta}^{m}\left(\mathbf{R}^{q} \times \mathbf{R}^{p}\right)$. As usual we write $S_{\rho}^{m}\left(\mathbf{R}^{q} \times \mathbf{R}^{p}\right)$ in the case $\rho=1-\delta, \frac{1}{2} \leq \rho<1$, and $S^{m}\left(\mathbf{R}^{q} \times \mathbf{R}^{p}\right)$ in the case $\rho=1, \delta=0$. We shall denote by $\mathcal{M}_{k} S_{\rho, \delta}^{m}\left(\mathbf{R}^{q} \times \mathbf{R}^{p}\right)$ the space of $k \times k$ matrices with entries in $S_{\rho, \delta}^{m}\left(\mathbf{R}^{q} \times \mathbf{R}^{p}\right)$. By $\mathcal{M}_{k}(\mathbf{C})$, we denote the space of $k \times k$ matrices with

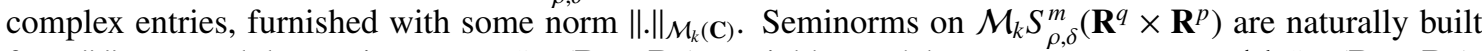
from $\|.\|_{\mathcal{M}_{k}(\mathbf{C})}$ and the seminorms on $S_{\rho, \delta}^{m}\left(\mathbf{R}^{q} \times \mathbf{R}^{p}\right)$. It yields a Fréchet space structure on $\mathcal{M}_{k} S_{\rho, \delta}^{m}\left(\mathbf{R}^{q} \times \mathbf{R}^{p}\right)$. In the case of matrix symbols, we shall also use the notation simplifications given above in the case $\rho=1-\delta$, and the case $\rho=1, \delta=0$.

We shall sometimes use the notion of multiple symbols. We set $\langle\xi ; \eta\rangle=\sqrt{1+|\xi|^{2}+|\eta|^{2}}$. A function $a(x, \xi, y, \eta) \in \mathscr{C}^{\infty}\left(\mathbf{R}^{q_{1}} \times \mathbf{R}^{p_{1}} \times \mathbf{R}^{q_{2}} \times \mathbf{R}^{p_{2}}\right)$ is in $S_{\rho, \delta}^{m, m^{\prime}}\left(\mathbf{R}^{q_{1}} \times \mathbf{R}^{p_{1}} \times \mathbf{R}^{q_{2}} \times \mathbf{R}^{p_{2}}\right), 0<\rho \leq 1,0 \leq \delta<1$, if for all multi-indices $\alpha_{1}, \beta_{1}, \alpha_{2}, \beta_{2}$, there exists $C_{\alpha_{1} \alpha_{2}}^{\beta_{1} \beta_{2}}>0$ such that

$$
\left|\partial_{x}^{\alpha_{1}} \partial_{\xi}^{\beta_{1}} \partial_{y}^{\alpha_{2}} \partial_{\eta}^{\beta_{2}} a(x, \xi, y, \eta)\right| \leq C_{\alpha_{1} \alpha_{2}}^{\beta_{1} \beta_{2}}\langle\xi\rangle^{m-\rho\left|\beta_{1}\right|+\delta\left|\alpha_{1}\right|}\langle\xi ; \eta\rangle^{\delta\left|\alpha_{2}\right|}\langle\eta\rangle^{m^{\prime}-\rho\left|\beta_{2}\right|}, \quad x \in \mathbf{R}^{q_{1}}, y \in \mathbf{R}^{q_{2}}, \xi \in \mathbf{R}^{p_{1}}, \eta \in \mathbf{R}^{p_{2}}
$$

(See for instance [23, Chapter 2]). For an example of a multiple symbol, we can consider functions of the form $p_{1}(x, \xi) p_{2}(x, y, \xi, \eta) p_{3}(y, \eta)$, with each term being a symbol w.r.t. its variables.

We shall use, in a standard way, the notation \# for the composition of symbols of pseudodifferential operators ( $\psi \mathrm{DO})$. In the case of matrix symbols, $a \# b$ will naturally denote the matrix symbol with entries $(a \# b)_{i j}=\sum_{k} a_{i k} \# b_{k j}$. When given an amplitude $p(x, y, \xi) \in \mathcal{M}_{k} S_{\rho, \delta}^{m}\left(X \times X \times \mathbf{R}^{n}\right), \rho \geq \delta$, we shall also use the notation $\sigma\{p\}(x, \xi)$ for the symbol of the $\psi$ DO with amplitude $p$. For $p \in \mathcal{M}_{k} S_{\rho, \delta}^{m}\left(X \times \mathbf{R}^{n}\right)$ we shall write $p^{*}$ for the symbol of the adjoint operator.

For $r \in \mathbf{R}$ we let $E^{(r)}$ be the $\psi \mathrm{DO}$ with symbol $\langle\xi\rangle^{r}$. The operator $E^{(r)}$ maps $H^{(s)}(X)$ onto $H^{(s-r)}(X)$ unitarily for all $s \in \mathbf{R}$ with $E^{(-r)}$ being the inverse map. We shall use the same notation for the diagonal operator that maps $\left(H^{(s)}(X)\right)^{k}$ onto $\left(H^{(s-r)}(X)\right)^{k}$ unitarily, $k \in \mathbf{N}^{*}$.

\section{Some results on Fourier integral operators in the scalar case}

\subsection{A class of Fourier integral operators with complex phase}

Let us consider the amplitude $h_{1}\left(z, x^{\prime}, x, \xi\right)=-i k_{1}\left(z, x^{\prime}, x, \xi\right)+l_{1}\left(z, x^{\prime}, x, \xi\right)$ that satisfies the following assumption.

Assumption 1.1. The amplitudes $k_{1}(z,$.$) and l_{1}\left(z\right.$, .) are in $\mathscr{C}^{0}\left([0, Z], S^{1}\left(X^{\prime} \times X \times \mathbf{R}^{n}\right)\right)$, are real valued, homogeneous of degree one in $\xi$, for $|\xi| \geq 1$, and $l_{1}(z,) \geq$.0 .

Let $z^{\prime}, z \in[0, Z]$, with $z^{\prime} \geq z$, and let $\Delta:=z^{\prime}-z$. Define the complex function $\phi_{\left(z^{\prime}, z\right)} \in \mathscr{C}^{\infty}\left(X^{\prime} \times X \times \mathbf{R}^{n}\right)$ by

$$
\phi_{\left(z^{\prime}, z\right)}\left(x^{\prime}, x, \xi\right):=\left\langle x^{\prime}-x \mid \xi\right\rangle+i \Delta h_{1}\left(z, x^{\prime}, x, \xi\right)=\left\langle x^{\prime}-x \mid \xi\right\rangle+\Delta k_{1}\left(z, x^{\prime}, x, \xi\right)+i \Delta l_{1}\left(z, x^{\prime}, x, \xi\right),
$$

and define the complex function $\tilde{\phi}_{\left(z^{\prime}, z\right)}\left(x^{\prime}, x, \xi\right)$, homogeneous of degree one, in $\xi$, for all $\xi \neq 0$, equal to $\phi_{\left(z^{\prime}, z\right)}\left(x^{\prime}, x, \xi\right)$ when $|\xi|=1$.

Lemma 1.2. There exists $\Delta_{1}>0$ such that $\tilde{\phi}_{\left(z^{\prime}, z\right)}$ is a non-degenerate phase function, of positive type, for $0 \leq \Delta=z^{\prime}-z \leq \Delta_{1}$, at any point $\left(x_{0}^{\prime}, x_{0}, \xi_{0}\right)$ where $\partial_{\xi} \tilde{\phi}_{\left(z^{\prime}, z\right)}=0$.

Proof. We denote by $\tilde{k}_{1}$ and $\tilde{l}_{1}$ the homogeneous extension of $k_{1}$ and $l_{1}$ for all $\xi \neq 0$. Note that $\operatorname{Im}\left(\tilde{\phi}_{\left(z^{\prime}, z\right)}\right) \geq 0$ and thus $\partial_{\xi} \tilde{\phi}_{\left(z^{\prime}, z\right)}=0$ implies $\tilde{l}_{1}\left(z, x^{\prime}, x, \xi\right)=0$ by Euler's formula. Let us consider a point $\gamma_{0}=\left(x_{0}^{\prime}, x_{0}, \xi_{0}\right)$ where $d \tilde{\phi}_{\left(z^{\prime}, z\right)}=0$. Then $\tilde{l}_{1}$ vanishes at this point $\gamma_{0}$ and so do all its partial differentials w.r.t. $x^{\prime}, x$ and $\xi$. 
We note that $\partial_{x^{\prime}} \tilde{\phi}_{\left(z^{\prime}, z\right)}\left(\gamma_{0}\right)=\xi_{0}+\Delta \partial_{x^{\prime}} \tilde{k}_{1}\left(z, x_{0}^{\prime}, x_{0}, \xi_{0}\right)=0$. Then, because of homogeneity, for $\Delta$ sufficiently small, $\xi_{0}=0$ is the only possible solution, since

$$
\sup _{\substack{z \in[0, Z] \\ z}} \sup _{\substack{x^{\prime} \in X^{\prime} \\ k \in X \\ \xi \xi \mid=1}} \partial_{x^{\prime}} \tilde{k}_{1}\left(z, x^{\prime}, x, \xi\right) \leq C .
$$

Thus $d \tilde{\phi}_{\left(z^{\prime}, z\right)}$ does not vanish in $X^{\prime} \times X \times\left(\mathbf{R}^{n} \backslash 0\right)$ for $\Delta$ sufficiently small. If we now consider the partial differentials of $\partial_{\xi_{1}} \tilde{\phi}_{\left(z^{\prime}, z\right)}, \ldots, \partial_{\xi_{n}} \tilde{\phi}_{\left(z^{\prime}, z\right)}$ w.r.t. to $x_{1}, \ldots, x_{n}$,

$$
\partial_{x_{i}} \partial_{\xi_{j}} \tilde{\phi}_{\left(z^{\prime}, z\right)}\left(x^{\prime}, x, \xi\right)=\delta_{i j}+\Delta \partial_{x_{i}} \partial_{\xi_{j}} \tilde{k}_{1}\left(z, x^{\prime}, x, \xi\right),
$$

we see that the differentials $d \partial_{\xi_{1}} \tilde{\phi}_{\left(z^{\prime}, z\right)}, \ldots, d \partial_{\xi_{n}} \tilde{\phi}_{\left(z^{\prime}, z\right)}$ are linearly independent for $\Delta$ sufficiently small, since $\partial_{x_{i}} \partial_{\xi_{j}} \tilde{k}_{1}\left(z, x^{\prime}, x, \xi\right), 1 \leq i, j \leq n$, are bounded w.r.t. $z, x^{\prime}$ and $x$, and homogeneous of degree zero in $\xi$.

Remark 1.3. In an abusive way, we shall say that the function $\phi_{\left(z^{\prime}, z\right)}$ is a complex phase function of positive type. The function $\phi_{\left(z^{\prime}, z\right)}$ is assumed to be homogeneous of degree one in $\xi$ only when $|\xi| \geq 1$. This however is not an obstacle to the subsequent analysis, e.g., FIO properties, since to define such operators the phase function need not be homogeneous of degree one for small $|\xi|$. With the use of cut-off functions in the amplitude of the operators below, one can in fact modify the phase functions for small $|\xi|$.

Proposition 25.4.4 in [14] assesses that a non-degenerate phase function $\varphi(y, \theta) \in \mathscr{C}^{\infty}\left(Y \times \mathbf{R}^{N}\right)$, of positive type at some point $\left(y_{0}, \theta_{0}\right)$, locally defines a Lagrangian ideal of functions. In the case where there exists a Lagrangian ideal $K$, such that in the neighborhood of every point of $K_{\mathbf{R}}$ the ideal is generated by the same phase function $\varphi(x, \theta)$, and conversely, the ideal of functions locally generated by $\varphi$ coincides with $K$ (at a point $\left(y_{0}, \eta_{0}\right) \in T^{*}(Y) \backslash 0$ associated to a point $\left(y_{0}, \theta_{0}\right) \in Y \times \mathbf{R}^{N}$ where $\varphi$ is stationary in the sense of Proposition 25.4.4 in [14]), we shall say that the phase function $\varphi(x, \theta)$ globally defines $K$ or that it is a global phase function. We recall that if $K$ is an ideal of functions on $T^{*}(Y) \backslash 0$, we set

$$
K_{\mathbf{R}}=\left\{(y, \eta) \in T^{*}(Y) \backslash 0 ; \forall u \in K, u(y, \eta)=0\right\} .
$$

Because of the usual twist $\left(x^{\prime}, \xi^{\prime}, x, \xi\right) \mapsto\left(x^{\prime}, \xi^{\prime}, x,-\xi\right)$ (see Section 25.2 and 25.5 in [14]), we use the symplectic 2-form $\sigma^{\prime}-\sigma$ on $T^{*}\left(X^{\prime} \times X\right)$, where $\sigma^{\prime}$ and $\sigma$ are the symplectic 2-forms on $T^{*}\left(X^{\prime}\right)$ and $T^{*}(X)$ respectively. Twisted Lagrangian ideals are called canonical ideals.

Proposition 1.4. There exists $\Delta_{2}>0$ such that if $z^{\prime}, z \in[0, Z]$ with $0 \leq \Delta=z^{\prime}-z \leq \Delta_{2}$ then the phase function $\phi_{\left(z^{\prime}, z\right)}$ is a global phase function.

Proof. For $\left(x^{\prime}, x, \theta\right) \in X^{\prime} \times X \times \mathbf{R}^{n}$, set

$$
\varphi\left(x^{\prime}, x, \theta\right)=\operatorname{Re}\left(\phi_{\left(z^{\prime}, z\right)}\right)\left(x^{\prime}, x, \theta\right)=\left\langle x^{\prime}-x \mid \theta\right\rangle+\Delta k_{1}\left(z, x^{\prime}, x, \theta\right)
$$

which is a real non-degenerate phase function. The stationary point set for $\varphi\left(x^{\prime}, x, \theta\right)$, given by

$$
\Sigma_{\varphi}=\left\{\left(x^{\prime}, x, \theta\right) \in X^{\prime} \times X \times\left(\mathbf{R}^{n} \backslash 0\right) ; \partial_{\theta} \varphi\left(x^{\prime}, x, \theta\right)=0\right\},
$$

is thus a submanifold. Define

$$
C_{\varphi}=\left\{\left(x^{\prime}, \xi^{\prime}, x, \xi\right) \in T^{*}\left(X^{\prime}\right) \times T^{*}(X) ; \exists \theta \in \mathbf{R}^{n}, \partial_{\theta} \varphi\left(x^{\prime}, x, \theta\right)=0, \quad \xi^{\prime}=\partial_{x^{\prime}} \varphi\left(x^{\prime}, x, \theta\right), \xi=-\partial_{x} \varphi\left(x^{\prime}, x, \theta\right)\right\}
$$

Observe that the equations

$$
\xi^{\prime}=\partial_{x^{\prime}} \varphi\left(x^{\prime}, x, \theta\right)=\theta+\Delta \partial_{x^{\prime}} k_{1}\left(x^{\prime}, x, \theta\right), \quad x^{\prime}-x+\Delta \partial_{\theta} k_{1}\left(x^{\prime}, x, \theta\right)=0
$$

yield a global homogeneous diffeomorphism $\left(x^{\prime}, \xi^{\prime}\right)=f(x, \theta)$ for $\Delta$ sufficiently small, by a Banach fixedpoint argument, since $\partial_{x^{\prime}} k_{1}\left(x^{\prime}, x, \theta\right)$ and $\partial_{\theta} k_{1}\left(x^{\prime}, x, \theta\right)$ are bounded on $X^{\prime} \times X \times \mathcal{S}^{n-1}$. Similarly we obtain that the equation

$$
\xi=-\partial_{x^{\prime}} \varphi\left(x^{\prime}, x, \theta\right)=\theta-\Delta \partial_{x} k_{1}\left(x^{\prime}, x, \theta\right)
$$


also defines a global diffeomorphism $(x, \xi)=g(x, \theta)$ for $\Delta$ sufficiently small. Thus, for $\Delta$ sufficiently small, the set $C_{\varphi}$ is the graph of a smooth canonical transformation (hence, a submanifold). Let us denote by $\alpha$ the natural global isomorphism between $\Sigma_{\varphi}$ and $C_{\varphi}$. Define

$$
\begin{aligned}
\Sigma_{\phi} & :=\Sigma_{\varphi} \cap\left\{\left(x^{\prime}, x, \theta\right) \in X^{\prime} \times X \times\left(\mathbf{R}^{n} \backslash 0\right) ; l_{1}\left(x^{\prime}, x, \theta\right)=0\right\}, \\
\mathcal{K}_{\mathbf{R}} & :=\alpha\left(\Sigma_{\phi}\right) \subset\left(T^{*}\left(X^{\prime}\right) \times T^{*}(X)\right) \backslash 0 .
\end{aligned}
$$

The set $\mathcal{K}_{\mathbf{R}}$ is thus part of a (smooth) submanifold.

Let $\gamma=\left(x^{\prime}, x, \theta\right) \in \Sigma_{\phi}$. To any conic neighborhood $V_{\gamma}$ of $\gamma$ in $X^{\prime} \times X \times \mathbf{R}^{n}$ corresponds, through $\alpha$, a conic neighborhood $U_{v}$ in $\left(T^{*}\left(X^{\prime}\right) \times T^{*}(X)\right) \backslash 0$ of $v=\alpha(\gamma)=\left(x^{\prime}, \xi, x, \xi^{\prime}\right) \in \mathcal{K}_{\mathbf{R}}$. We choose $V_{\gamma}$ sufficiently small to apply Proposition 25.4.4 in [14]. In the neighborhood $U_{v}$, the phase function $\phi_{\left(z^{\prime}, z\right)}\left(x^{\prime}, x, \theta\right)$ thus defines a canonical ideal that we denote by $J_{v}$.

We now define the ideal $K$ of functions on $\left(T^{*}\left(X^{\prime}\right) \times T^{*}(X)\right) \backslash 0$ defined by the set of $\mathscr{C}^{\infty}$ functions $u$ such that for all $v \in \mathcal{K}_{\mathbf{R}}$, and for all $\chi \in \mathscr{C}_{c}^{\infty}\left(U_{v}\right)$, we have $\chi u \in J_{v}$. We see that $K$ is a canonical ideal (see properties (i)-(iii) in Definition 24.4.1 in [14]) and we have $K_{\mathbf{R}}=\mathcal{K}_{\mathbf{R}}$. At every point of $K_{\mathbf{R}}$ the canonical ideal $K$ is thus locally defined by $\phi_{\left(z^{\prime}, z\right)}\left(x^{\prime}, x, \theta\right)$, which concludes the proof.

Let us denote by $J_{\left(z^{\prime}, z\right)}$ the canonical ideal globally defined by $\phi_{\left(z^{\prime}, z\right)}$. Considering an amplitude $\sigma_{\left(z^{\prime}, z\right)}\left(x^{\prime}, x, \xi\right)$ bounded w.r.t. $z$ and $z^{\prime}$ with values in $S_{\rho}^{m}\left(X^{\prime} \times X \times \mathbf{R}^{n}\right), \frac{1}{2} \leq \rho \leq 1$, we define the distribution kernel in $\mathscr{D}^{\prime}\left(X^{\prime} \times X\right)$

$$
A_{\left(z^{\prime}, z\right)}\left(x^{\prime}, x\right)=\int e^{i\left\langle x^{\prime}-x \mid \xi\right\rangle} e^{-\Delta h_{1}\left(z, x^{\prime}, x, \xi\right)} \sigma_{\left(z^{\prime}, z\right)}\left(x^{\prime}, x, \xi\right) d \xi=\int e^{i \phi_{\left(z^{\prime}, z\right)}\left(x^{\prime}, x, \xi\right)} \sigma_{\left(z^{\prime}, z\right)}\left(x^{\prime}, x, \xi\right) d \xi,
$$

where $\Delta=z^{\prime}-z, 0 \leq z \leq z^{\prime} \leq Z$, as an oscillatory integral (see Section 7.8 in [16]). The associated operator, $\mathcal{A}_{\left(z^{\prime}, z\right)}$ is a local FIO [12,9]. Because of Proposition 1.4, we have the following proposition.

Proposition 1.5. The operator $\mathcal{A}_{\left(z^{\prime}, z\right)}$ is a global FIO with complex phase in $\mathbf{R}^{n}$, for $\Delta$ sufficiently small. Its kernel is in $I^{0}\left(X^{\prime} \times X,\left(J_{\left(z^{\prime}, z\right)}\right)^{\prime}, \Omega_{X^{\prime} \times X}^{\frac{1}{2}}\right)$.

We denote the half density bundle on $X^{\prime} \times X$ by $\Omega_{X^{\prime} \times X}^{1 / 2}$. Note that $\left(J_{\left(z^{\prime}, z\right)}\right)^{\prime}$ stands for the twisted canonical ideal, i.e. a Lagrangian ideal (see Section 25.5 in [14]).

Proof. In view of Definitions 24.5.9 and 25.5.1 in [14], and arguing as in the proof of Proposition 1.4, we see that the canonical ideal $J_{\left(z^{\prime}, z\right)}$, globally defined by $\phi_{\left(z^{\prime}, z\right)}$ satisfies $J_{\left(z^{\prime}, z\right), \mathbf{R}} \subset T^{*}\left(X^{\prime}\right) \backslash 0 \times T^{*}(X) \backslash 0$, which yields the conclusion.

\subsection{Action on $\mathscr{P}\left(\mathbf{R}^{n}\right)$ and $\mathscr{P}^{\prime}\left(\mathbf{R}^{n}\right)$}

We shall study the properties of the operator $\mathcal{A}_{\left(z^{\prime}, z\right)}$ for small values of the parameter $\Delta$.

Composition of global FIOs usually requires the operators to be properly supported. For the composition of $\mathcal{A}_{\left(z^{\prime}, z\right)}$ with $\mathcal{A}_{\left(z^{\prime \prime \prime}, z^{\prime \prime}\right)}$ or with its adjoint, we need not make such a requirement because of the following proposition which is proven in Appendix A.

Proposition 1.6. There exists $\Delta_{3}>0$ such that if $z^{\prime}, z \in[0, Z]$ with $0 \leq \Delta=z^{\prime}-z \leq \Delta_{3}$, then $\mathcal{A}_{\left(z^{\prime}, z\right)}$ maps $\mathscr{P}\left(\mathbf{R}^{n}\right)$ into $\mathscr{P}\left(\mathbf{R}^{n}\right)$ and $\mathscr{P}^{\prime}\left(\mathbf{R}^{n}\right)$ into $\mathscr{P}^{\prime}\left(\mathbf{R}^{n}\right)$ continuously. Furthermore, the map $\left(\sigma_{\left(z^{\prime}, z\right)}, u\right) \mapsto \mathcal{A}_{\left(z^{\prime}, z\right)}(u)$ is continuous.

\subsection{Some calculus results}

We shall need the following lemma.

Lemma 1.7. Let $p_{s}(y, \eta)$ be bounded w.r.t. the parameter $s$ with values in $S_{\rho^{\prime}}^{m}\left(Y \times \mathbf{R}^{r}\right)$, $\frac{1}{2} \leq \rho^{\prime} \leq 1$, and define

$$
\tilde{\eta}_{s}(\Delta, y, \eta):=\eta-\Delta f_{s}(y, \eta)
$$


where $f_{s}$ is bounded w.r.t. the parameter $s$ with values in $S^{1}\left(Y \times \mathbf{R}^{r}, \mathbf{R}^{r}\right)$ and homogeneous of degree one in $\eta$, for $|\eta| \geq 1$. Then

$$
\tilde{p}_{s}(\Delta, y, \eta):=p_{s}(y, \tilde{\eta}(\Delta, y, \eta))
$$

is bounded w.r.t. $s$ and $\Delta$ with values in $S_{\rho^{\prime}}^{m}\left(Y \times \mathbf{R}^{r}\right), 0 \leq \Delta \leq \Delta_{4}$, for $\Delta_{4}>0$ sufficiently small. In the case $\rho^{\prime}=1$ it is bounded w.r.t. $s$ with values in $\mathscr{C}^{\infty}\left(\left[0, \Delta_{4}\right], S^{m}\left(Y \times \mathbf{R}^{r}\right)\right)$.

Proof. Let $\Delta_{4}$ be small enough such that $\left|\eta-\Delta f_{s}(y, \eta)\right| \geq C_{0}>0$ if $|\eta|=1$ and $\Delta \in\left[0, \Delta_{4}\right]$. We then have

$$
1+C_{0}|\eta| \leq 1+\left|\eta-\Delta f_{s}(y, \eta)\right| \leq 1+C_{1}|\eta|, \eta \in \mathbf{R}^{r},|\eta| \geq 1, \Delta \in\left[0, \Delta_{4}\right] .
$$

This inequality yields the proper estimates for $\partial_{y}^{\alpha} \partial_{\eta}^{\beta} \tilde{p}_{s}$ to prove that $\tilde{p}_{s} \in S_{\rho^{\prime}}^{m}\left(\mathbf{R}^{p} \times \mathbf{R}^{r}\right)$. Bounds w.r.t. to the parameters $s$ and $\Delta$ come naturally. In the case $\rho^{\prime}=1$, derivatives w.r.t. $\Delta$ do not affect the symbol order and type. The proof is complete.

Lemma 1.8. Let $q(z, y, \eta)$ be a non-negative symbol, bounded w.r.t. $z$ with values in $S^{1}\left(Y \times \mathbf{R}^{r}\right)$. Then, $e^{-\Delta q(z, y, \eta)}$ is bounded w.r.t. $\Delta$ and $z$ with values in $S_{\frac{1}{2}}^{0}\left(Y \times \mathbf{R}^{r}\right)$

A proof is provided in Section 2.3 below.

Remark 1.9. In fact, one can obtain the symbol $e^{-\Delta q(z, y, \eta)}$ to be with values in $S_{\rho^{\prime}}^{0}\left(Y \times \mathbf{R}^{r}\right)$, with $\frac{1}{2} \leq \rho^{\prime}<1$, by making some special choices for the symbol $q(z, y, \eta)$ (see [29, Section 2] and Section 2.3 below).

In the sequel, in this section we shall thus assume that $e^{-\Delta l_{1}(z, y, \eta)}$ takes values in $S_{\rho^{\prime}}^{0}\left(Y \times \mathbf{R}^{r}\right)$, for some $\rho^{\prime}$, $\frac{1}{2} \leq \rho^{\prime}<1$. We set $\delta^{\prime}=1-\rho^{\prime}$. We have the following proposition.

Proposition 1.10. Let $p(x, \xi) \in S_{\rho^{\prime \prime}, \delta^{\prime \prime}}^{m^{\prime}}\left(X \times \mathbf{R}^{n}\right)$, with $0 \leq \delta^{\prime \prime} \leq \frac{1}{2} \leq \rho^{\prime \prime} \leq 1$. Then, for $\Delta$ sufficiently small:

1. The operator $p\left(x, D_{x}\right) \circ \mathcal{A}_{\left(z^{\prime}, z\right)}$, can be put in the form

$$
\left(p\left(x, D_{x}\right) \circ \mathcal{A}_{\left(z^{\prime}, z\right)}\right)(u)\left(x^{\prime}\right)=\iint e^{i\left\langle x^{\prime}-x \mid \xi\right\rangle} e^{i \Delta k_{1}\left(z, x^{\prime}, x, \xi\right)} q_{\left(z^{\prime}, z\right)}\left(x^{\prime}, x, \xi\right) u(x) d \xi d x
$$

with $q_{\left(z^{\prime}, z\right)}\left(x^{\prime}, x, \xi\right)$ bounded w.r.t. to $z^{\prime}$ and $z$ with values in $S_{\min \left(\rho, \rho^{\prime}, \rho^{\prime \prime}\right), \max \left(\delta, \delta^{\prime}, \delta^{\prime \prime}\right)}^{m+m^{\prime}}\left(X^{\prime} \times X \times \mathbf{R}^{n}\right)$ given by

$$
q_{\left(z^{\prime}, z\right)}\left(x^{\prime}, x, \xi\right)=\iint e^{-i\left\langle x^{\prime}-y \mid \xi-\eta\right\rangle} e^{-\Delta l_{1}(z, y, x, \xi)} e^{i \Delta\left(k_{1}(z, y, x, \xi)-k_{1}\left(z, x^{\prime}, x, \xi\right)\right)} p\left(x^{\prime}, \eta\right) \sigma_{\left(z^{\prime}, z\right)}(y, x, \xi) d \eta d y .
$$

2. The operator $\mathcal{A}_{\left(z^{\prime}, z\right)} \circ p\left(x, D_{x}\right)$, can be put in the form

$$
\left(\mathcal{A}_{\left(z^{\prime}, z\right)} \circ p\left(x, D_{x}\right)\right)(u)\left(x^{\prime}\right)=\iint e^{i\left\langle x^{\prime}-x \mid \xi\right\rangle} e^{i \Delta k_{1}\left(z, x^{\prime}, x, \xi\right)} q_{\left(z^{\prime}, z\right)}\left(x^{\prime}, x, \xi\right) u(x) d \xi d x
$$

with $q_{\left(z^{\prime}, z\right)}\left(x^{\prime}, x, \xi\right)$ bounded w.r.t. to $z^{\prime}$ and $z$ with values in $S_{\min \left(\rho, \rho^{\prime}, \rho^{\prime \prime}\right), \max \left(\delta, \delta^{\prime}, \delta^{\prime \prime}\right)}^{m+m^{\prime}}\left(X^{\prime} \times X \times \mathbf{R}^{n}\right)$ given by

$$
q_{\left(z^{\prime}, z\right)}\left(x^{\prime}, x, \xi\right)=\iint e^{i\langle x-y \mid \xi-\eta\rangle} e^{-\Delta l_{1}\left(z, x^{\prime}, y, \xi\right)} e^{i \Delta\left(k_{1}\left(z, x^{\prime}, y, \xi\right)-k_{1}\left(z, x^{\prime}, x, \xi\right)\right)} \sigma_{\left(z^{\prime}, z\right)}\left(x^{\prime}, y, \xi\right) p(y, \eta) d \eta d y .
$$

Formulae (1.3)-(1.4) for the amplitudes are to be understood as oscillatory integrals. Note that the form of formula (1.4) differs from that of formula (2.3) in Theorem 10.2.1 in [23]. Recall however that, for operators of this form, there is no unique choice for the amplitude.

The proof of Item 1 can be adapted in a straightforward manner from that of Theorem 10.2.2 in [23] for $\Delta$ sufficiently small. Note that the variable $x$ in (1.3) will then just act as an additional parameter but does not modify the argumentation of the proof. In Appendix A we only prove Item 2 of the proposition, with a method close to that of the proof of Theorem 10.2.2 in [23].

Remark 1.11. In fact, the proof of Proposition 1.10 shows that we can replace $p\left(x^{\prime}, \eta\right) \sigma_{\left(z^{\prime}, z\right)}(y, x, \xi)$ in $(1.3)$ (resp. $\sigma_{\left(z^{\prime}, z\right)}\left(x^{\prime}, y, \xi\right) p(y, \eta)$ in $\left.(1.4)\right)$ by any multiple $\operatorname{symbol}^{1} \rho_{\left(z^{\prime}, z\right)}\left(x^{\prime}, y, x, \xi, \eta\right)$ in $S_{\min \left(\rho^{\prime}, \rho^{\prime \prime}\right), \max \left(\delta^{\prime}, \delta^{\prime \prime}\right)}^{m, m^{\prime}}$ and the two oscillatory integrals (1.4), (1.3) yield symbols in $S_{\min \left(\rho, \rho^{\prime}, \rho^{\prime \prime}\right), \max \left(\delta, \delta^{\prime}, \delta^{\prime \prime}\right)}^{m+m^{\prime}}\left(X^{\prime} \times X \times \mathbf{R}^{n}\right)$.

\footnotetext{
${ }^{1}$ With the same boundedness w.r.t. to $z^{\prime}$ and $z$ and with $x$ as a smooth parameter in (1.3), and $x^{\prime}$ as a smooth parameter in (1.4).
} 


\subsection{Sobolev space regularity}

We now turn to global $L^{2}$ and Sobolev space regularity for operators of the form of $\mathcal{A}_{\left(z^{\prime}, z\right)}$ in (1.2).

Theorem 1.12. Let $s \in \mathbf{R}$. There exist $C \geq 0$ and $\Delta_{5}>0$ such that

$$
\left\|\mathcal{A}_{\left(z^{\prime}, z\right)}\right\|_{\left(H^{(s)}, H^{(s-m)}\right)} \leq C p\left(\sigma_{\left(z^{\prime}, z\right)}\right),
$$

for all $z^{\prime}, z \in[0, Z]$, and $0 \leq \Delta=z^{\prime}-z \leq \Delta_{5}$, where $p($.$) is some appropriately chosen seminorm on$ $S_{\rho}^{m}\left(X^{\prime} \times X \times \mathbf{R}^{n}\right)$.

Sobolev norm estimation results often have a local character in the literature (see e.g. [13, 14]). The result we prove here gives a global Sobolev regularity estimate. It generalizes some results found in [22, 23], where the phase function is real and in a form close to that used here but only depend on the variables $x^{\prime}$ and $\xi$. Other global estimates for FIOs can be found for instance in $[35,1]$. Note that the real part of the phase of $\mathcal{A}_{\left(z^{\prime}, z\right)}$ satisfies conditions (A-I)-(A-III) of [1] for $\Delta$ sufficiently small. In the case $m=0$, if the symbol $\sigma_{\left(z^{\prime}, z\right)}$ were of type 1,0 (or more generally of type 0,0$)$ and the phase function were to be real $\left(l_{1}(z,)=0.\right)$, then, we could apply the regularity result of [1] (see also [3]). Here, because of the symbol type and the complex phase function we use, we need to prove this regularity result. In the proof, we shall take advantage of the structure of the phase function.

Proof. We first treat the case in which $\sigma_{\left(z^{\prime}, z\right)}\left(x^{\prime}, x, \xi\right)$ is bounded w.r.t. to $z^{\prime}$ and $z$ with values in $S_{\rho}^{0}\left(X^{\prime} \times X \times\right.$ $\mathbf{R}^{n}$ ) and prove $L^{2}$ continuity.

The adjoint operator of $\mathcal{A}_{\left(z^{\prime}, z\right)}$ is given by

$$
\mathcal{A}_{\left(z^{\prime}, z\right)}^{*}(u)\left(x^{\prime}\right)=\iint e^{i\left\langle x^{\prime}-x \mid \xi\right\rangle} e^{-\Delta \bar{h}_{1}\left(z, x, x^{\prime}, \xi\right)} \bar{\sigma}_{\left(z^{\prime}, z\right)}\left(x, x^{\prime}, \xi\right) u(x) d \xi d x,
$$

from which we find

$$
\begin{aligned}
\mathcal{F}(u)\left(x^{\prime}\right):=\mathcal{A}_{\left(z^{\prime}, z\right)} \circ \mathcal{A}_{\left(z^{\prime}, z\right)}^{*}(u)\left(x^{\prime}\right) & =\iint e^{i\left\langle x^{\prime}-x \mid \xi\right\rangle}\left(\iint e^{i\langle y-x \mid \eta-\xi\rangle}\right. \\
& \left.\times e^{-\Delta\left(h_{1}\left(z, x^{\prime}, y, \xi\right)+\bar{h}_{1}(z, x, y, \eta)\right)} \sigma_{\left(z^{\prime}, z\right)}\left(x^{\prime}, y, \xi\right) \bar{\sigma}_{\left(z^{\prime}, z\right)}(x, y, \eta) d \eta d y\right) u(x) d \xi d x .
\end{aligned}
$$

With the lemma below we obtain

$$
\mathcal{F}(u)\left(x^{\prime}\right)=\iint e^{i\left\langle x^{\prime}-x \mid \xi\right\rangle} f_{\left(z^{\prime}, z\right)}\left(x^{\prime}, x, \xi\right) u(x) d \xi d x,
$$

with $f_{\left(z^{\prime}, z\right)}\left(x^{\prime}, x, \xi\right)$ bounded w.r.t. $z^{\prime}$ and $z$ with values in $S_{\min \left(\rho, \rho^{\prime}\right)}^{0}\left(X^{\prime} \times X \times \mathbf{R}^{n}\right)$, for $\Delta$ sufficiently small. Furthermore, for any seminorm $p$ on $S_{\min \left(\rho, \rho^{\prime}\right)}^{0}$, there exists a seminorm $q$ on $S_{\rho}^{0}$ such that we have $p\left(f_{\left(z^{\prime}, z\right)}\right) \leq$ $C q\left(\sigma_{\left(z^{\prime}, z\right)}\right)^{2}$. Thus, for $\Delta$ sufficiently small, $\mathcal{F}$ is in $\Psi_{\min \left(\rho, \rho^{\prime}\right)}^{0}$ and bounded on $L^{2}$ by the Calderón-Vaillancourt Theorem (see [23, Chapter 7, Sections 1,2] or [39, Section XIII-2]):

$$
\left\|\mathcal{A}_{\left(z^{\prime}, z\right)} \circ \mathcal{A}_{\left(z^{\prime}, z\right)}^{*}\right\|_{\left(L^{2}, L^{2}\right)} \leq C q\left(\sigma_{\left(z^{\prime}, z\right)}\right)^{2},
$$

where $q$ is a properly chosen seminorm on $S_{\rho}^{0}\left(X^{\prime} \times X \times \mathbf{R}^{n}\right)$. The result thus follows in this first case.

We now consider the amplitude $\sigma_{\left(z^{\prime}, z\right)}\left(x^{\prime}, x, \xi\right)$ to be bounded w.r.t. to $z^{\prime}$ and $z$ with values in $S_{\rho}^{m}\left(X^{\prime} \times X \times \mathbf{R}^{n}\right)$. We compose the operator $\mathcal{A}_{\left(z^{\prime}, z\right)}$ from the left by $E^{(s-m)}$ and from the right by $E^{(-s)}$. By Proposition 1.10, we obtain an operator with a similar form (with a real phase) with an amplitude bounded w.r.t. to $z^{\prime}$ and $z$ with values in $S_{\min \left(\rho, \rho^{\prime}\right)}^{0}\left(X^{\prime} \times X \times \mathbf{R}^{n}\right)$, to which we apply the first part of the proof.

Lemma 1.13. The operator $\mathcal{F}$ is of the form

$$
\mathcal{F}(u)\left(x^{\prime}\right)=\iint e^{i\left\langle x^{\prime}-x \mid \xi\right\rangle} f_{\left(z^{\prime}, z\right)}\left(x^{\prime}, x, \xi\right) u(x) d \xi d x,
$$

with $f_{\left(z^{\prime}, z\right)}\left(x^{\prime}, x, \xi\right)$ bounded w.r.t. $z^{\prime}$ and $z$ with values in $S_{\min \left(\rho, \rho^{\prime}\right)}^{0}\left(X^{\prime} \times X \times \mathbf{R}^{n}\right)$, for $\Delta$ sufficiently small. Furthermore, for a seminorm $p$ on $S_{\min \left(\rho, \rho^{\prime}\right)}^{0}$, there exists a seminorm $q$ on $S_{\rho}^{0}$ such that we have

$$
p\left(f_{\left(z^{\prime}, z\right)}\right) \leq C q\left(\sigma_{\left(z^{\prime}, z\right)}\right)^{2} .
$$

The proof of Lemma 1.13 can be found in Appendix A. 


\section{The thin-slab propagator for a symmetric system}

\subsection{The Cauchy problem}

Let $k, n \in \mathbf{N}$. Let $s \in \mathbf{R}$ and $Z>0$. We consider the Cauchy problem

$$
\begin{aligned}
\partial_{z} u+a\left(z, x, D_{x}\right) u & =0, \quad 0<z \leq Z, \\
\left.u\right|_{z=0} & =u_{0} \in\left(H^{(s+1)}\left(\mathbf{R}^{n}\right)\right)^{k},
\end{aligned}
$$

where the matrix symbol $a(z, x, \xi)$ takes values in $\mathcal{M}_{k} S^{1}\left(X \times \mathbf{R}^{n}\right)$ with $z$ as a parameter.

In this section, we focus on the symmetric case. More precisely, we shall make the following assumption.

Assumption 2.1. The principal matrix symbol of $a, a_{1}(z, x, \xi)=-i b_{1}(z, x, \xi)+c_{1}(z, x, \xi)$, is such that both $b_{1}$ and $c_{1}$ are continuous w.r.t. $z$ with values in $\mathcal{M}_{k} S^{1}\left(X \times \mathbf{R}^{n}\right)$ and homogeneous of degree one in $\xi$, for $|\xi| \geq 1$. Furthermore, they are hermitian symmetric and $c_{1}(z, x, \xi)$ is non-negative.

We set the remaining part of the symbol $a(z, x, \xi)$ as $a_{0}(z, x, \xi), a_{0}(z, x, \xi):=a(z, x, \xi)-a_{1}(z, x, \xi)$, which is assumed to be continuous w.r.t. $z$ with values in $\mathcal{M}_{k} S^{0}\left(X \times \mathbf{R}^{n}\right)$.

Adapting the proof of Lemma 23.1.1 in [15] to systems (making use of the sharp Gårding inequality for positive first-order matrix symbols [28, Theorem 3.2], [40], [39, Chapter VII]) for any function in

$$
V:=\mathscr{C}^{1}\left([0, Z],\left(H^{(s)}\left(\mathbf{R}^{n}\right)\right)^{k}\right) \cap \mathscr{C}^{0}\left([0, Z],\left(H^{(s+1)}\left(\mathbf{R}^{n}\right)\right)^{k}\right),
$$

we have the following energy estimate (see also [4, Theorem 6.4.3])

$$
\sup _{z \in[0, Z]}\|u(z, .)\|_{\left(H^{(s)}\right)^{k}} \leq C\|u(0, .)\|_{\left(H^{(s)}\right)^{k}}+C \int_{0}^{Z}\left\|\partial_{z} u+a\left(z, x, D_{x}\right) u\right\|_{\left(H^{(s))^{k}}\right.} d z .
$$

Then, there exists a unique solution in $V$ to the Cauchy problem (2.1)-(2.2) ([15, Theorem 23.1.2], [4, Theorem 6.4.5]).

By Proposition 9.3 in [10, Chapter VI] the family of operators $\left(a\left(z, x, D_{x}\right)\right)_{z \in[0, Z]}$ generates a strongly continuous evolution system. Let $U\left(z^{\prime}, z\right)$ denote the corresponding evolution system:

$$
U\left(z^{\prime \prime}, z^{\prime}\right) \circ U\left(z^{\prime}, z\right)=U\left(z^{\prime \prime}, z\right), \quad Z \geq z^{\prime \prime} \geq z^{\prime} \geq z \geq 0,
$$

with

$$
\begin{aligned}
\partial_{z} U\left(z, z_{0}\right)\left(u_{0}\right)+a\left(z, x, D_{x}\right) U\left(z, z_{0}\right)\left(u_{0}\right) & =0, \quad 0 \leq z_{0}<z \leq Z, \\
U\left(z_{0}, z_{0}\right)\left(u_{0}\right) & =u_{0} \in\left(H^{(s+1)}\left(\mathbf{R}^{n}\right)\right)^{k},
\end{aligned}
$$

while $U\left(z, z_{0}\right)\left(u_{0}\right) \in\left(H^{(s+1)}\left(\mathbf{R}^{n}\right)\right)^{k}$ for all $z \in\left[z_{0}, Z\right]$. For the Cauchy problem (2.1)-(2.2) we take $z_{0}=0$.

We shall make the following further assumption on $a(z, x, \xi)$.

Assumption 2.2. There exists $w(z, x, \xi)$ continuous w.r.t. $z$ with values in $\mathcal{M}_{k} S^{0}\left(X \times \mathbf{R}^{n}\right)$, unitary, homogeneous of degree zero in $\xi$, for $|\xi| \geq 1$, such that

$$
b_{1}(z, x, \xi)=w(z, x, \xi) d_{b}(z, x, \xi)(w(z, x, \xi))^{-1}, \quad c_{1}(z, x, \xi)=w(z, x, \xi) d_{c}(z, x, \xi)(w(z, x, \xi))^{-1},
$$

where $d_{b}(z, x, \xi)$ and $d_{c}(z, x, \xi)$ are $k \times k$ diagonal matrices with entries continuous w.r.t. $z$ with values in $S^{1}\left(\mathbf{R}^{n} \times \mathbf{R}^{n}\right)$ and homogeneous of degree one in $\xi$, for $|\xi| \geq 1$. The diagonal entries of $d_{c}(z, x, \xi)$ are non-negative since $c_{1}(z, x, \xi) \geq 0$.

This assumption is sometimes referred to as having a "geometrically regular" matrix symbol $a(z, x, \xi)$ (see e.g. [33, Definition 2.2 (ii)]). 
Assumption 2.2 will be satisfied for instance if the eigenvalues of $b_{1}(z, x, \xi)$ and $c_{1}(z, x, \xi)$ have constant multiplicities ${ }^{2}$ since $b_{1}(z, x, \xi)$ and $c_{1}(z, x, \xi)$ is hermitian symmetric [19, Section II.4] and if the matrices $b_{1}(z, x, \xi)$ and $c_{1}(z, x, \xi)$ commute. However, Assumption 2.2 is much more general and allows for the crossing of smooth eigenvalues.

We set $v(z, x, \xi):=(w(z, x, \xi))^{-1}=(w(z, x, \xi))^{*}$ and $d(z, x, \xi):=-i d_{b}(z, x, \xi)+d_{c}(z, x, \xi)$. We shall denote by $d_{b, l}(z, x, \xi)$ and $d_{c, l}(z, x, \xi), 1 \leq l \leq k$, the diagonal entries of the matrices $d_{b}(z, x, \xi)$ and $d_{c}(z, x, \xi)$.

Example 2.3. The Dirac operator $\sum_{j=1}^{3} \alpha_{j} D_{x_{j}}+m \beta$ has an hermitian symmetric principal symbol. Here the Dirac matrices are $4 \times 4$ matrices and are given by

$$
\alpha_{j}=\left(\begin{array}{cc}
0 & \sigma_{j} \\
\sigma_{j} & 0
\end{array}\right), \quad j=1,2,3, \quad \text { with } \sigma_{1}=\left(\begin{array}{cc}
0 & 1 \\
1 & 0
\end{array}\right), \sigma_{2}=\left(\begin{array}{cc}
0 & -i \\
i & 0
\end{array}\right), \sigma_{3}=\left(\begin{array}{cc}
1 & 0 \\
0 & -1
\end{array}\right) .
$$

The two eigenvalues $\pm|\xi|$ both have constant multiplicity two. A norm convergence result of a Trotterproduct formula for the Dirac operator can be found in [17].

\subsection{The thin-slab propagator}

Let $0 \leq z \leq z^{\prime} \leq Z$. We set, for $\Delta=z^{\prime}-z$,

$$
g_{\left(z^{\prime}, z\right)}(x, \xi):=e^{-\Delta a_{0}(z, x, \xi)} .
$$

The function $g_{\left(z^{\prime}, z\right)}(x, \xi)$ is bounded w.r.t. $z$ and smooth w.r.t. $\Delta$ with values in $\mathcal{M}_{k} S^{0}\left(X \times \mathbf{R}^{n}\right)$ by the following lemma.

Lemma 2.4. Let $q_{z}(y, \eta)$ be bounded w.r.t. $z$ with values in $\mathcal{M}_{k} S^{0}\left(Y \times \mathbf{R}^{p}\right)$. Then $p_{\Delta, z}(y, \eta)=e^{\Delta q_{z}(y, \eta)}$ is bounded w.r.t. $z$ and smooth w.r.t. $\Delta$ with values in $\mathcal{M}_{k} S^{0}\left(Y \times \mathbf{R}^{p}\right)$.

Proof. The exponential function for matrices is bounded. In fact, we have

$$
\left\|e^{\Delta q_{z}(y, \eta)}\right\|_{\mathcal{M}_{k}(\mathbf{C})} \leq e^{\Delta\left\|q_{z}(y, \eta)\right\|_{\mathcal{M}_{k}(\mathbf{C})}} \leq C, \quad y \in Y, \eta \in \mathbf{R}^{p}, z \in[0, Z],
$$

if we choose $\|.\|_{\mathcal{M}_{k}(\mathbf{C})}$ to be the operator norm on $\mathcal{M}_{k}(\mathbf{C})$. We now estimate $\left\|\partial_{y_{i}} p_{\Delta, z}(y, \eta)\right\|_{\mathcal{M}_{k}(\mathbf{C})}$, for $1 \leq i \leq n$, and note that

$$
\partial_{y} p_{\Delta, z}(y, \eta)=\Delta \int_{0}^{1} e^{s \Delta q_{z}(y, \eta)} \partial_{y} q_{z}(y, \eta) e^{(1-s) \Delta q_{z}(y, \eta)} d s
$$

We thus obtain

$$
\left\|\partial_{y} p_{\Delta, z}(y, \eta)\right\|_{\mathcal{M}_{k}(\mathbf{C})} \leq C, \quad y \in Y, \eta \in \mathbf{R}^{p} .
$$

The estimate for $\left\|\partial_{\eta_{i}} p_{\Delta, z}(y, \eta)\right\|_{\mathcal{M}_{k}(\mathbf{C})}$, for $1 \leq i \leq n$ follows the same. More generally the estimate for $\left\|\partial_{y}^{\alpha} \partial_{\eta}^{\beta} p_{\Delta, z}(y, \eta)\right\|_{\mathcal{M}_{k}(\mathbf{C})}$, for arbitrary multi-indices $\alpha$ and $\beta$ is obtained by induction. Finally, smoothness w.r.t. to $\Delta$ and boundedness w.r.t. $z$ are clear with the above estimates.

Remark 2.5. By the proof of Lemma 18.1 .10 in [15], the previous lemma can in fact be generalized to $F\left(q_{z}(y, \eta)\right)$ for any function $F$ acting on $\mathcal{M}_{k}(\mathbf{C})$ such that $F(M)=\left(F_{11}(M), \ldots, F_{k k}(M)\right)$, with $F_{i j}(M)=$ $\tilde{F}_{i j}\left(M_{11}, \ldots, M_{k k}\right)$ in $\mathscr{C}^{\infty}\left(\mathbf{C}^{k \times k}, \mathbf{C}\right), 1 \leq i, j \leq k$.

We define the following $(k \times k$ matrix) kernel

$$
G_{\left(z^{\prime}, z\right)}\left(x^{\prime}, x\right):=\int e^{i\left\langle x^{\prime}-x \mid \xi\right\rangle} g_{\left(z^{\prime}, z\right)}\left(x^{\prime}, \xi\right) e^{-\Delta a_{1}\left(z, x^{\prime}, \xi\right)} d \xi
$$

\footnotetext{
${ }^{2}$ in particular if the eigenvalues are simple, i.e., in the strictly hyperbolic case.
} 
Such a kernel is well defined since we can write

$$
G_{\left(z^{\prime}, z\right)}\left(x^{\prime}, x\right)=\int e^{i\left\langle x^{\prime}-x \mid \xi\right\rangle} g_{\left(z^{\prime}, z\right)}\left(x^{\prime}, \xi\right) w\left(z, x^{\prime}, \xi\right) e^{-\Delta d\left(z, x^{\prime}, \xi\right)} v\left(z, x^{\prime}, \xi\right) d \xi
$$

and thus, each entry of the matrix kernel is a finite sum of scalar kernels of the form of (1.2). Each component of the associated operator is thus a finite sum of FIOs of the form of $\mathcal{A}_{\left(z^{\prime}, z\right)}$ in Section 1 . We therefore have the following regularity results from Proposition 1.6 and Theorem 1.12.

Proposition 2.6. Let $s \in \mathbf{R}$. For $\Delta$ sufficiently small, the operator $\mathcal{G}_{\left(z^{\prime}, z\right)}$ with distribution kernel $G_{\left(z^{\prime}, z\right)}\left(x^{\prime}, x\right)$, is a continuous map of $\left(\mathscr{P}\left(\mathbf{R}^{n}\right)\right)^{k}$ into $\left(\mathscr{P}\left(\mathbf{R}^{n}\right)\right)^{k},\left(\mathscr{P}^{\prime}\left(\mathbf{R}^{n}\right)\right)^{k}$ into $\left(\mathscr{P}^{\prime}\left(\mathbf{R}^{n}\right)\right)^{k}$, and $\left(H^{(s)}\left(\mathbf{R}^{n}\right)\right)^{k}$ into $\left(H^{(s)}\left(\mathbf{R}^{n}\right)\right)^{k}$. In particular, there exists $C \geq 0$ such that

$$
\left\|\mathcal{G}_{\left(z^{\prime}, z\right)}\right\|_{\left(\left(H^{(s)}\right)^{k},\left(H^{(s)}\right)^{k}\right)} \leq C
$$

for all $z^{\prime}, z \in[0, Z], \Delta=z^{\prime}-z$.

Let $m \in \mathbf{R}$. If the matrix symbol $g_{\left(z^{\prime}, z\right)}$ is changed into a function bounded w.r.t. $\Delta$ and $z$ with values in $\mathcal{M}_{k} S_{\rho^{\prime}}^{m}\left(\mathbf{R}^{n} \times \mathbf{R}^{n}\right), \rho^{\prime} \in\left[\frac{1}{2}, 1\right]$, then the associated operator maps $\left(H^{(s)}\left(\mathbf{R}^{n}\right)\right)^{k}$ into $\left(H^{(s-m)}\left(\mathbf{R}^{n}\right)\right)^{k}$ continuously, with a uniform operator-norm estimate as above.

In the sequel, we shall say that operators of the form of $\mathcal{G}_{\left(z^{\prime}, z\right)}$ are FIOs with the complex matrix phase

$$
\phi_{\left(z^{\prime}, z\right)}\left(x^{\prime}, x, \xi\right)=\left\langle x^{\prime}-x \mid \xi\right\rangle+b_{1}\left(z, x^{\prime}, \xi\right)+i c_{1}\left(z, x^{\prime}, \xi\right) .
$$

We aim to give a more precise estimate of the Sobolev operator norm of the thin-slab propagator in $L\left(\left(H^{(s)}\left(\mathbf{R}^{n}\right)\right)^{k},\left(H^{(s)}\left(\mathbf{R}^{n}\right)\right)^{k}\right)$. We shall in fact obtain

$$
\left\|\mathcal{G}_{\left(z^{\prime}, z\right)}\right\|_{\left(\left(H^{(s)}\right)^{k},\left(H^{(s)}\right)^{k}\right)} \leq 1+M \Delta, \quad \Delta=z^{\prime}-z,
$$

for some $M \geq 0$ and for $\Delta$ sufficiently small (Theorem 2.22). To obtain such an estimate we need to understand the properties of the matrix symbol $e^{-\Delta c_{1}(x, \xi)}$ when $\Delta$ is small.

\subsection{A class of symbols}

We first introduce some definitions.

Definition 2.7. Let $L \geq 2$. A (scalar) symbol $q(z,$.$) bounded w.r.t. z$ with values in $S^{1}\left(\mathbf{R}^{p} \times \mathbf{R}^{r}\right)$ is said to satisfy Property $\left(P_{L}\right)$ if it is non-negative and satisfies

$\left(P_{L}\right) \quad\left|\partial_{y}^{\alpha} \partial_{\eta}^{\beta} q(z, y, \eta)\right| \leq C\langle\eta\rangle^{-|\beta|+(|\alpha|+|\beta|) / L}(1+q(z, y, \eta))^{1-(|\alpha|+|\beta|) / L}, \quad z \in[0, Z], y \in \mathbf{R}^{p}, \eta \in \mathbf{R}^{r}$.

We then set $\rho=1-1 / L$ and $\delta=1 / L$.

Examples of symbols with such a property with $L>2$ are given in [37]. In fact we have the following lemma [29].

Lemma 2.8. Let $q(z, y, \eta)$ be bounded w.r.t. $z$ with values in $S^{1}\left(\mathbf{R}^{p} \times \mathbf{R}^{r}\right)$. If $q \geq 0$ then $q$ satisfies Prop$\operatorname{erty}\left(P_{L}\right)$ for $L=2$.

Remark 2.9. If the symbol $q(z, y, \eta)$ and $p(z, y, \eta)$ both satisfy Property $\left(P_{L}\right)$ then the amplitude $q\left(z, y^{\prime}, \eta\right)+$ $p(z, y, \eta)$ also satisfies Property $\left(P_{L}\right)$ (with derivatives w.r.t. $y, y^{\prime}$ and $\eta$ ).

The following definition concerns matrix symbols.

Definition 2.10. Let $L \geq 2, \rho=1-1 /$ L and $\delta=1 /$ L. Let $\rho_{\Delta}(z, y, \eta)$ be a function in $\mathscr{C}^{\infty}\left(Y \times \mathbf{R}^{r}, \mathcal{M}_{k}(\mathbf{C})\right)$ depending on the parameters $\Delta \geq 0$ and $z \in[0, Z]$. We say that $\rho_{\Delta}$ satisfies Property $\left(Q_{L}\right)$ if the following holds

$\left(Q_{L}\right) \quad \partial_{y}^{\alpha} \partial_{\eta}^{\beta}\left(\rho_{\Delta}-\left.\rho_{\Delta}\right|_{\Delta=0}\right)(z, y, \eta)=\Delta^{m+\delta(|\alpha|+|\beta|)} \rho_{\Delta}^{m \alpha \beta}(z, y, \eta)$, for $|\alpha|+|\beta| \leq L, \quad 0 \leq m \leq 1-\delta(|\alpha|+|\beta|)$,

where $\rho_{\Delta}^{m \alpha \beta}(z, y, \eta)$ is bounded w.r.t. $\Delta$ and $z$ with values in $\mathcal{M}_{k} S_{\rho}^{m-\rho|\beta|+\delta|\alpha|}\left(Y \times \mathbf{R}^{r}\right)$. It follows that $\rho_{\Delta}(z, y, \eta)-$ $\left.\rho_{\Delta}\right|_{\Delta=0}(z, y, \eta)$ is itself bounded w.r.t. $\Delta$ and $z$ with values in $\mathcal{M}_{k} S_{\rho}^{0}\left(Y \times \mathbf{R}^{r}\right)$. 
In [29], the following three lemmata, are proven in the scalar case, $k=1$.

Lemma 2.11. Let $q(z,$.$) be bounded w.r.t. z$ with values in $S^{1}\left(Y \times \mathbf{R}^{r}\right)$ and satisfy Property $\left(P_{L}\right)$. Define $\rho_{\Delta}(z, y, \eta)=e^{-\Delta q(z, y, \eta)}$. Then $\rho_{\Delta}$ satisfies Property $\left(Q_{L}\right), k=1$, for $\Delta \in\left[0, \Delta_{\mathfrak{F}}\right]$ for any $\Delta_{\mathfrak{F}}>0$. As $\left.\rho_{\Delta}\right|_{\Delta=0}=1, \rho_{\Delta}$ is itself bounded w.r.t. $\Delta$ and $z$ with values in $S_{\rho}^{0}\left(Y \times \mathbf{R}^{r}\right)$.

Lemma 2.12. Let $f \in \mathscr{C}^{\infty}(\mathbf{R})$ and $\rho_{\Delta}(z, y, \eta)$ in $\mathscr{C}^{\infty}\left(Y \times \mathbf{R}^{r}\right)$ that satisfies Property $\left(Q_{L}\right), k=1$, and such that $\left.\rho_{\Delta}(z,)\right|_{.\Delta=0}$ is independent of $y$ and $\eta$. Then $f\left(\rho_{\Delta}\right)(z, y, \eta)$ satisfies Property $\left(Q_{L}\right)$.

Lemma 2.13. Let $\rho_{\Delta}(z, y, \eta) \in S_{\rho}^{0}\left(Y \times \mathbf{R}^{r}\right)$ satisfy Property $\left(Q_{L}\right), k=1$, such that $\left.\rho_{\Delta}\right|_{\Delta=0}$ is constant. Let $f_{\Delta}(z, y, \eta)$ be bounded w.r.t. $z$ and $\Delta$ with values in $S^{1}\left(Y \times \mathbf{R}^{r}\right)$ be homogeneous of degree one in $\eta$ for $|\eta| \geq 1$. Define $\tilde{\eta}(\Delta, z, y, \eta):=\eta-\Delta f_{\Delta}(z, y, \eta)$. Then $\tilde{\rho}_{\Delta}(z, y, \eta):=\rho_{\Delta}(z, y, \tilde{\eta}(\Delta, z, y, \eta))$ satisfies Property $\left(Q_{L}\right), k=1$, for $\Delta$ sufficiently small.

Remark 2.14. These three lemmata naturally extend to diagonal matrix symbols and we shall use them in this form below.

Proposition 2.15. Let $L \geq 2$. Let $\rho_{\Delta}(z, y, \eta)$ be bounded w.r.t. $z$ and $\Delta$ with values in $\mathcal{M}_{k} S_{\rho}^{0}\left(Y \times \mathbf{R}^{r}\right)$ that satisfies Property $\left(Q_{L}\right), L \geq 2, \rho=1-1 /$ L. Let $r(z,$.$) be bounded w.r.t. z$ with values in $\mathcal{M}_{k} S^{0}\left(Y \times \mathbf{R}^{n}\right)$. Then $\left(r \rho_{\Delta}\right)(z, y, \eta)$ and $\left(\rho_{\Delta} r\right)(z, y, \eta)$ both satisfy Property $\left(Q_{L}\right)$.

Proof. Let $\alpha, \beta$ be multi-indices such that $|\alpha|+|\beta| \leq L$ and let $m$ be such that $0 \leq m \leq 1-\delta(|\alpha|+|\beta|)$. When computing $\partial_{y}^{\alpha} \partial_{\eta}^{\beta} r\left(\rho_{\Delta}-\left.\rho_{\Delta}\right|_{\Delta=0}\right)$ we obtain a sum of terms of the form

$$
\partial_{y}^{\alpha_{1}} \partial_{\eta}^{\beta_{1}} r(z, y, \eta) \partial_{y}^{\alpha_{2}} \partial_{\eta}^{\beta_{2}}\left(\rho_{\Delta}-\left.\rho_{\Delta}\right|_{\Delta=0}\right)(z, y, \eta),
$$

with $\alpha_{1}+\alpha_{2}=\alpha$ and $\beta_{1}+\beta_{2}=\beta$. We choose $m^{\prime}=m+\delta\left(\left|\alpha_{1}\right|+\left|\beta_{1}\right|\right)$. We have $0 \leq m^{\prime} \leq 1-\delta\left(\left|\alpha_{2}\right|+\left|\beta_{2}\right|\right)$. By Property $\left(Q_{L}\right)$, we write

$$
\partial_{y}^{\alpha_{2}} \partial_{\eta}^{\beta_{2}}\left(\rho_{\Delta}-\left.\rho_{\Delta}\right|_{\Delta=0}\right)(z, y, \eta)=\Delta^{m^{\prime}+\delta\left(\left|\alpha_{2}\right|+\left|\beta_{2}\right|\right)} \rho_{\Delta}^{m^{\prime} \alpha_{2} \beta_{2}}(z, y, \eta),
$$

with $\rho_{\Delta}^{m^{\prime} \alpha_{2} \beta_{2}}(z, y, \eta)$ bounded w.r.t. $z$ with values in $\mathcal{M}_{k} S^{m^{\prime}-\rho\left|\beta_{2}\right|+\delta\left|\alpha_{2}\right|}\left(Y \times \mathbf{R}^{r}\right)$. Thus, we have

$$
\partial_{y}^{\alpha_{1}} \partial_{\eta}^{\beta_{1}} r(z, y, \eta) \partial_{y}^{\alpha_{2}} \partial_{\eta}^{\beta_{2}}\left(\rho_{\Delta}-\left.\rho_{\Delta}\right|_{\Delta=0}\right)(z, y, \eta)=\Delta^{m+\delta(|\alpha|+|\beta|)}\left(\partial_{y}^{\alpha_{1}} \partial_{\eta}^{\beta_{1}} r\right) \rho_{\Delta}^{m^{\prime} \alpha_{2} \beta_{2}}(z, y, \eta)
$$

and $\left(\partial_{y}^{\alpha_{1}} \partial_{\eta}^{\beta_{1}} r\right) \rho_{\Delta}^{m^{\prime} \alpha_{2} \beta_{2}}(z, y, \eta)$ is bounded w.r.t. $\Delta$ and $z$ with values in $\mathcal{M}_{k} S_{\rho}^{p}\left(Y \times \mathbf{R}^{r}\right)$ with

$$
p=m^{\prime}-\rho\left|\beta_{2}\right|+\delta\left|\alpha_{2}\right|-\left|\beta_{1}\right|=m+\delta\left(\left|\alpha_{1}\right|+\left|\alpha_{2}\right|\right)+(\delta-1)\left|\beta_{1}\right|-\rho\left|\beta_{2}\right|=m+\delta|\alpha|-\rho|\beta|,
$$

since $\delta=1-\rho$. The proof for $\rho_{\Delta} r$ can be carried out similarly.

Note that the type 1,0 of the matrix symbol $r(z,$.$) was of importance in the proof.$

Corollary 2.16. Assume that the entries of the diagonal matrix symbol $d_{c}(z, x, \xi)$ satisfy Property $\left(P_{L}\right)$, for $L \geq 2$. Then the matrix symbol $e^{-\Delta c_{1}(z, x, \xi)}$ satisfies Property $\left(Q_{L}\right)$ in $\mathcal{M}_{k} S_{\rho}^{0}\left(X \times \mathbf{R}^{n}\right)$.

Recall that by Lemma 2.8, by default, the entries of the diagonal matrix symbol $d_{c}(z, x, \xi)$ satisfy Property $\left(P_{L}\right)$, for $L=2$.

Proof. The matrix symbol $e^{-\Delta d_{c}(z, x, \xi)}$ satisfies Property $\left(Q_{L}\right)$, from Lemma 2.11 and Remark 2.14. Then by Proposition 2.15, the matrix symbol $e^{-\Delta c_{1}(z, x, \xi)}=w(z, x, \xi) e^{-\Delta d_{c}(z, x, \xi)} v(z, x, \xi)$ satisfies Property $\left(Q_{L}\right)$.

Lemma 2.17. Let the matrix amplitudes $\rho_{\Delta}(z, x, y, \xi)$ and $\mu_{\Delta}(z, x, y, \xi)$ both satisfy Property $\left(Q_{L}\right)$ and be such that $\left.\rho_{\Delta}(z,)\right|_{.\Delta=0}$ and $\left.\mu_{\Delta}(z,)\right|_{.\Delta=0}$ are constant. Then the amplitude $\rho_{\Delta}(z, x, y, \xi) \mu_{\Delta}(z, x, t, \xi)$ satisfies Property $\left(Q_{L}\right)$ (with differentials w.r.t. $x, y, t$, and $\xi$ ). 
Proof. Let $0 \leq m \leq 1$. We write

$$
\begin{aligned}
v_{\Delta}(z, x, y, t, \xi) & :=\rho_{\Delta}(z, x, y, \xi) \mu_{\Delta}(z, x, t, \xi)-\left.\left.\rho_{\Delta}\right|_{\Delta=0} \mu_{\Delta}\right|_{\Delta=0}(z) \\
& =\rho_{\Delta}(z, x, y, \xi)\left(\mu_{\Delta}(z, x, t, \xi)-\left.\mu_{\Delta}\right|_{\Delta=0}(z)\right)+\left(\rho_{\Delta}(z, x, y, \xi)-\left.\rho_{\Delta}\right|_{\Delta=0}(z)\right)\left(\left.\mu_{\Delta}\right|_{\Delta=0}(z)\right) \\
& =\Delta^{m} \rho_{\Delta}(z, x, y, \xi) \mu_{\Delta}^{m 00}(z, x, t, \xi)+\Delta^{m} \rho_{\Delta}^{m 00}(z, x, y, \xi)\left(\left.\mu_{\Delta}\right|_{\Delta=0}(z)\right)
\end{aligned}
$$

where $\mu_{\Delta}^{m 00}(z, x, t, \xi)$ and $\rho_{\Delta}^{m 00}(z, x, y, \xi)$ are bounded w.r.t. $\Delta$ and $z$ with values in $\mathcal{M}_{k} S_{\rho}^{m}$ by Property $\left(Q_{L}\right)$. Since $\left.\mu_{\Delta}\right|_{\Delta=0}(z)$ solely depends on $z$ and is bounded and $\rho_{\Delta}(z, x, y, \xi)$ is itself bounded w.r.t. $\Delta$ and $z$ with values in $\mathcal{M}_{k} S_{\rho}^{0}$, we obtain Property $\left(Q_{L}\right)$ when no differentiation is applied.

Let $\alpha, \alpha^{\prime}, \alpha^{\prime \prime}$ and $\beta$ be mutli-indices such that $\lambda=|\alpha|+\left|\alpha^{\prime}\right|+\left|\alpha^{\prime \prime}\right|+|\beta| \leq L$ and let $0 \leq m \leq 1-\delta \lambda$. Computing $\partial_{x}^{\alpha} \partial_{y}^{\alpha^{\prime}} \partial_{t}^{\alpha^{\prime \prime}} \partial_{\xi}^{\beta} v_{\Delta}(z, x, y, t, \xi)$ we obtain a linear combination of terms of the form

$$
\partial_{x}^{\alpha_{1}} \partial_{y}^{\alpha^{\prime}} \partial_{\xi}^{\beta_{1}} \rho_{\Delta}(z, x, y, \xi) \partial_{x}^{\alpha_{2}} \partial_{t}^{\alpha^{\prime \prime}} \partial_{\xi}^{\beta_{2}} \mu_{\Delta}(z, x, t, \xi), \quad \alpha=\alpha_{1}+\alpha_{2}, \beta=\beta_{1}+\beta_{2},
$$

which, using Property $\left(Q_{L}\right)$, we write

$$
\Delta^{m^{\prime}+\delta\left(\left|\alpha_{1}\right|+\left|\alpha^{\prime}\right|+\left|\beta_{1}\right|\right)+m^{\prime \prime}+\delta\left(\left|\alpha_{2}\right|+\left|\alpha^{\prime \prime}\right|+\left|\beta_{2}\right|\right)} \rho_{\Delta}^{m^{\prime}\left(\alpha_{1}, \alpha^{\prime}\right) \beta_{1}}(z, x, y, \xi) \mu_{\Delta}^{m^{\prime \prime}\left(\alpha_{2}, \alpha^{\prime \prime}\right) \beta_{2}}(z, x, t, \xi),
$$

with $\rho_{\Delta}^{m^{\prime}\left(\alpha_{1}, \alpha^{\prime}\right) \beta_{1}}$ and $\mu_{\Delta}^{m^{\prime \prime}\left(\alpha_{2}, \alpha^{\prime \prime}\right) \beta_{2}}$ bounded w.r.t. $z$ and $\Delta$ with values in $M_{k} S_{\rho}^{m^{\prime}+\delta\left(\left|\alpha_{1}\right|+\left|\alpha^{\prime}\right|\right)-\rho\left|\beta_{1}\right|}\left(X \times Y \times \mathbf{R}^{n}\right)$ and $\mathcal{M}_{k} S_{\rho}^{m^{\prime \prime}+\delta\left(\left|\alpha_{2}\right|+\left|\alpha^{\prime \prime}\right|\right)-\rho\left|\beta_{2}\right|}\left(X \times T \times \mathbf{R}^{n}\right)$ respectively and where $0 \leq m^{\prime} \leq 1-\delta\left(\left|\alpha_{1}\right|+\left|\alpha^{\prime}\right|+\left|\beta_{1}\right|\right)$ and $0 \leq m^{\prime \prime} \leq 1-\delta\left(\left|\alpha_{2}\right|+\left|\alpha^{\prime \prime}\right|+\left|\beta_{2}\right|\right)$. We choose $m^{\prime}$ and $m^{\prime \prime}$ such that $m^{\prime}+m^{\prime \prime}=m$. Then, the considered term of $\partial_{x}^{\alpha} \partial_{y}^{\alpha^{\prime}} \partial_{t}^{\alpha^{\prime \prime}} \partial_{\xi}^{\beta} v_{\Delta}(z, x, y, t, \xi)$ is of the form

$$
\Delta^{m+\delta\left(|\alpha|+\left|\alpha^{\prime}\right|+\left|\alpha^{\prime \prime}\right|+|\beta|\right)} \rho_{\Delta}^{m^{\prime}\left(\alpha_{1}, \alpha^{\prime}\right) \beta_{1}}(z, x, y, \xi) \mu_{\Delta}^{m^{\prime \prime}\left(\alpha_{2}, \alpha^{\prime \prime}\right) \beta_{2}}(z, x, t, \xi),
$$

with $\rho_{\Delta}^{m^{\prime}\left(\alpha_{1}, \alpha^{\prime}\right) \beta_{1}}(z, x, y, \xi) \mu_{\Delta}^{m^{\prime \prime}\left(\alpha_{2}, \alpha^{\prime \prime}\right) \beta_{2}}(z, x, t, \xi)$ bounded w.r.t. $z$ and $\Delta$ with values in $\mathcal{M}_{k} S_{\rho}^{m+\delta\left(|\alpha|+\left|\alpha^{\prime}\right|+\left|\alpha^{\prime \prime}\right|\right)-\rho|\beta|}(X \times$ $\left.Y \times T \times \mathbf{R}^{n}\right)$.

Lemma 2.18. Let $\rho_{\Delta}(z, x, y, \xi)$ be an amplitude in $\mathcal{M}_{k} S_{\rho}^{0}\left(\mathbf{R}^{2 p} \times \mathbf{R}^{p}\right)$ depending on the parameters $\Delta \geq 0$ and $z \in[0, Z]$ that satisfies Property $\left(Q_{L}\right)$ for $1 \leq|\alpha|+|\beta| \leq 2$ and such that $\left.\rho_{\Delta}(z,)\right|_{.\Delta=0}$ is independent of $(x, y, \xi)$. Let $r(x, \xi) \in \mathcal{M}_{k} S^{s}\left(\mathbf{R}^{p} \times \mathbf{R}^{p}\right)$ for some $s \in \mathbf{R}$. Then

$$
\sigma\left\{\rho_{\Delta} r\right\}(z, x, \xi)-\rho_{\Delta}(z, x, x, \xi) r(x, \xi)=\Delta^{m+2 \delta} \lambda_{\Delta}^{m}(z, x, \xi), 0 \leq m \leq \rho-\delta,
$$

where the function $\lambda_{\Delta}^{m}(z, x, \xi)$ is bounded w.r.t. $\Delta$ and $z$ with values in $\mathcal{M}_{k} S_{\rho}^{m+s-(\rho-\delta)}\left(\mathbf{R}^{p} \times \mathbf{R}^{p}\right)$.

For a proof see the proof of Lemma 2.22 in [29], which can be directly adapted to the matrix case. We shall also need the following lemma which is a closely related results.

Lemma 2.19. Let $q_{\Delta}(z, x, \xi)$ be an symbol in $\mathcal{M}_{k} S_{\rho}^{0}\left(\mathbf{R}^{p} \times \mathbf{R}^{p}\right)$ depending on the parameters $\Delta \geq 0$ and $z \in[0, Z]$ that satisfies Property $\left(Q_{L}\right)$ for $|\alpha|=1$ and such that $\left.q_{\Delta}(z,)\right|_{.\Delta=0}$ is independent of $x$. Let $r_{z}(x, \xi)$ be bounded w.r.t. $z$ with values in $\mathcal{M}_{k} S^{s}\left(\mathbf{R}^{p} \times \mathbf{R}^{p}\right)$ for some $s \in \mathbf{R}$. Then

$$
\left(r_{z} \# q_{\Delta}\right)(z, x, \xi)-r_{z}(x, \xi) q_{\Delta}(z, x, \xi)=\Delta^{m+\delta} \lambda_{\Delta}^{m}(z, x, \xi), 0 \leq m \leq \rho,
$$

where the function $\lambda_{\Delta}^{m}(z, x, \xi)$ is bounded w.r.t. $\Delta$ and $z$ with values in $\mathcal{M}_{k} S_{\rho}^{m+s-\rho}\left(\mathbf{R}^{p} \times \mathbf{R}^{p}\right)$.

For a proof see the proof of Proposition 2.5 in [30], which can be directly adapted to the matrix case.

We shall need the following sharp Gårding inequality.

Theorem 2.20. Let $\frac{1}{2} \leq \rho \leq 1$ and set $\delta=1-\rho$. Let $p(x, \xi)$ be a $\mathscr{C}^{\infty} k \times k$ matrix that satisfies

$$
\begin{aligned}
& \forall x, \xi \in \mathbf{R}^{n} \times \mathbf{R}^{n}, \quad \forall f \in \mathbf{C}^{k}, \quad \operatorname{Re}\langle p(x, \xi) f \mid f\rangle \geq 0, \\
& \|p(x, \xi)\|_{\mathcal{M}_{k}(\mathbf{C})} \leq C\langle\xi\rangle, \\
& \left\|\partial_{x}^{\alpha} p(x, \xi)\right\|_{\mathcal{M}_{k}(\mathbf{C})} \leq C_{\alpha}\langle\xi\rangle, \quad|\alpha|=1, \quad\left\|\partial_{\xi}^{\beta} p(x, \xi)\right\|_{\mathcal{M}_{k}(\mathbf{C})} \leq C_{\beta}, \quad|\beta|=1,
\end{aligned}
$$


and

$$
\partial_{x}^{\alpha} \partial_{\xi}^{\beta} p(x, \xi) \in \mathcal{M}_{k} S_{\rho}^{\rho-\delta+\delta|\alpha|-\rho|\beta|}\left(X \times \mathbf{R}^{n}\right), \quad \text { for }|\alpha+\beta|=2 .
$$

Then there exists a non-negative constant $C$ such that

$$
\operatorname{Re}(p(x, D) u, u)_{\left(\left(L^{2}\right)^{k},\left(L^{2}\right)^{k}\right)} \geq-C\|u\|_{\left(L^{2}\right)^{k}}^{2}, \quad u \in\left(\mathscr{P}\left(\mathbf{R}^{n}\right)\right)^{k} .
$$

The constant $C$ can be chosen uniformly if the symbol p remains in a set such that the constants in (2.7)(2.8) are uniform and if $\partial_{x}^{\alpha} \partial_{\xi}^{\beta} p(x, \xi),|\alpha+\beta|=2$, remain in bounded domains of $\mathcal{M}_{k} S_{\rho}^{\rho-\delta+\delta|\alpha|-\rho|\beta|}\left(X \times \mathbf{R}^{n}\right)$ respectively.

In other words, for the partial differentiations of order zero and one the symbol $p$ behaves like an element of $\mathcal{M}_{k} S_{1,0}^{1}$ and like an element of $\mathcal{M}_{k} S_{\rho}^{\rho-\delta}$ for higher-order derivatives. Note that considering $p(x, \xi)$ to be in $\mathcal{M}_{k} S_{\rho}^{1}\left(X \times \mathbf{R}^{n}\right)$, we cannot directly apply the usual sharp Gårding inequality to obtain a lower $L^{2}$ bound when $\frac{1}{2} \leq \rho<1$.

Note that the hypothesis needed for the sharp Gårding inequality have been further weakened by J.-M. Bony [2]: only partial differentiations of orders greater than two are needed. His very elegant proof is however specialized to scalar symbols and cannot be adapted to the present system case. The proof of Theorem 2.20 can be found in Appendix A.

The following result is at the heart of the precise Sobolev operator-norm estimation of the thin-slab propagator $\mathcal{G}_{\left(z^{\prime}, z\right)}$.

Theorem 2.21. Let $k(z, x, \xi)$ be a $k \times k$ diagonal matrix symbol with non-negative entries, that satisfy Property $\left(P_{L}\right), L \geq 2$. Let $m(z, x, \xi)$ be a $k \times k$ unitary matrix symbol, such that $m(z, x, \xi)$ and $m(z, x, \xi)^{*}=$ $(m(z, x, \xi))^{-1}$ are both bounded w.r.t. $z$ with entries in $S^{0}\left(X \times \mathbf{R}^{n}\right)$. Set

$$
h(z, x, \xi)=m(z, x, \xi) k(z, x, \xi)(m(z, x, \xi))^{-1},
$$

The matrix symbol $\mu(z, x, \xi)=e^{-\Delta h(z, x, \xi)}$ is bounded w.r.t. $\Delta$ and $z$ with values in $\mathcal{M}_{k} S_{\rho}^{0}\left(X \times \mathbf{R}^{n}\right)$ and the pseudodifferential operator $\mathcal{A}_{\left(z^{\prime}, z\right)}=\mu\left(z, x, D_{x}\right)$ is continuous from $\left(L^{2}\left(\mathbf{R}^{n}\right)\right)^{k}$ into $\left(L^{2}\left(\mathbf{R}^{n}\right)\right)^{k}$. Moreover, there exist $\Delta_{7}>0$ and $M \geq 0$ such that $\mathcal{A}_{\left(z^{\prime}, z\right)}$ satisfies

$$
\left\|\mathcal{A}_{\left(z^{\prime}, z\right)}\right\|_{\left(\left(L^{2}\right)^{k},\left(L^{2}\right)^{k}\right)} \leq 1+M \Delta
$$

for all $z^{\prime}, z \in[0, Z]$ such that $0 \leq \Delta=z^{\prime}-z \leq \Delta_{7}$.

Proof. In the proof, we shall always assume that $\Delta$ is sufficiently small to apply the invoked properties and results. By Proposition 2.15, the symbols $\rho_{\Delta}(z, x, \xi)=e^{-\Delta k(z, x, \xi)}$ and $\mu_{\Delta}(z, x, \xi)=e^{-\Delta h(z, x, \xi)}$ both satisfy Property $\left(Q_{L}\right)$.

We prove that $\left(\mathcal{A}_{\left(z^{\prime}, z\right)} \circ \mathcal{A}_{\left(z^{\prime}, z\right)}^{*} u, u\right)_{\left(\left(L^{2}\right)^{k},\left(L^{2}\right)^{k}\right)} \leq(1+C \Delta)\|u\|_{\left(L^{2}\right)^{k}}^{2}$ for all $u \in\left(\mathscr{P}\left(\mathbf{R}^{n}\right)\right)^{k}$. The pseudodifferential operator $\mathcal{A}_{\left(z^{\prime}, z\right)} \circ \mathcal{A}_{\left(z^{\prime}, z\right)}^{*}$ has the matrix amplitude $p_{\Delta}(z, x, y, \xi)=e^{-\Delta h(z, x, \xi)} e^{-\Delta h(z, y, \xi)}$, which satisfies Property $\left(Q_{L}\right)$ by Lemma 2.17 . We then obtain

$$
\sigma\left\{p_{\Delta}(z, x, y, \xi)\right\}-e^{-2 \Delta h(z, x, \xi)}=\Delta \lambda_{\Delta}(z, x, \xi),
$$

where $\lambda_{\Delta}(z, x, \xi)$ is bounded w.r.t. $z$ and $\Delta$ with values in $\mathcal{M}_{k} S_{\rho}^{0}\left(X \times \mathbf{R}^{n}\right)$ by Lemma 2.18 (using $m=\rho-\delta$ ). By the Calderón-Vaillancourt Theorem (see [23, Chapter 7, Sections 1,2] or [39, Section XIII-2]), we shall obtain the desired estimate for $\left(\mathcal{A}_{\left(z^{\prime}, z\right)} \circ \mathcal{A}_{\left(z^{\prime}, z\right)}^{*} u, u\right)_{\left(\left(L^{2}\right)^{k},\left(L^{2}\right)^{k}\right)}$ if we prove $\operatorname{Re}\left(e^{-2 \Delta h\left(z, x, D_{x}\right)} u, u\right)_{\left(\left(L^{2}\right)^{k},\left(L^{2}\right)^{k}\right)} \leq$ $(1+C \Delta)\|u\|_{\left(L^{2}\right)^{k}}^{2}$ for all $u \in\left(\mathscr{P}\left(\mathbf{R}^{n}\right)\right)^{k}$.

We set $q_{\Delta}(z, x, \xi)=\left(1-e^{-2 \Delta h(z, x, \xi)}\right) / \Delta$ for $\Delta>0$ and observe that $q_{\Delta}(z, x, \xi)$ satisfies the conditions listed in Theorem 2.20 uniformly w.r.t. $z$ and $\Delta$. In fact, a first-order Taylor formula gives $\left\|q_{\Delta}(z, x, \xi)\right\|_{\mathcal{M}_{k}(\mathbf{C})} \leq C\langle\xi\rangle$. By Property $\left(Q_{L}\right)$ we obtain

$$
\left\|\partial_{x}^{\alpha} q_{\Delta}(z, x, \xi)\right\|_{\mathcal{M}_{k}(\mathbf{C})} \leq C\langle\xi\rangle, \quad|\alpha|=1, \quad\left\|\partial_{\xi}^{\beta} q_{\Delta}(z, x, \xi)\right\|_{\mathcal{M}_{k}(\mathbf{C})} \leq C, \quad|\beta|=1,
$$


using $m=\rho$ in $\left(Q_{L}\right)$ in both cases. Finally, if $|\alpha+\beta|=2$, we obtain that $\partial_{x}^{\alpha} \partial_{\xi}^{\beta} q_{\Delta}(z, x, \xi)$ is bounded uniformly w.r.t. $z$ and $\Delta$ with values in $\mathcal{M}_{k} S_{\rho}^{\rho-\delta+\delta|\alpha|-\rho|\beta|}\left(X \times \mathbf{R}^{n}\right)$ by choosing $m=\rho-\delta$ in $\left(Q_{L}\right)$.

By Theorem 2.20 we thus obtain $\operatorname{Re}\left(q_{\Delta}\left(z, x, D_{x}\right) u, u\right)_{\left.\left(L^{2}\right)^{k},\left(L^{2}\right)^{k}\right)} \geq-C\|u\|_{\left(L^{2}\right)^{k}}^{2}$ for all $u \in\left(\mathscr{P}\left(\mathbf{R}^{n}\right)\right)^{k}$ which yields

$$
\|u\|_{\left(L^{2}\right)^{k}}^{2}-\operatorname{Re}\left(e^{-2 \Delta h\left(z, x, D_{x}\right)} u, u\right)_{\left(\left(L^{2}\right)^{k},\left(L^{2}\right)^{k}\right)} \geq-C \Delta\|u\|_{\left(L^{2}\right)^{k}}^{2},
$$

which concludes the proof.

\subsection{Sobolev space regularity estimate for the thin-slab propagator}

We now state and prove the main theorem of this section, which will be essential to give a meaning to infinite products of operators of the form of $\mathcal{G}_{\left(z^{\prime}, z\right)}$ in Sections 3 and 4 .

Theorem 2.22. Let $s \in \mathbf{R}$. There exist $\Delta_{8}>0$ and $M \geq 0$ such that

$$
\left\|\mathcal{G}_{\left(z^{\prime}, z\right)}\right\|_{\left(\left(H^{(s)}\right)^{k},\left(H^{(s)}\right)^{k}\right)} \leq 1+M \Delta
$$

for all $z^{\prime}, z \in[0, Z]$ such that $0 \leq \Delta=z^{\prime}-z \leq \Delta_{8}$.

In the proof we assume that the diagonal entries of $d_{c}$ satisfy Property $\left(P_{L}\right)$ for some $L \geq 2$. We know that it is always true for $L=2$ by Lemma 2.8 but special choices for $c_{1}$ can be made. As before we use $\rho=1-1 / L$ and $\delta=1 / L$ with $\rho>\delta$ for $L>2$ and $\rho=\delta=\frac{1}{2}$ for $L=2$.

Proof. We first observe that we can write,

$$
g_{\left(z^{\prime}, z\right)}(x, \xi)=I_{k}+\Delta \tilde{g}_{\left(z^{\prime}, z\right)}(x, \xi),
$$

with $\tilde{g}_{\left(z^{\prime}, z\right)}$ bounded w.r.t. $\Delta$ and $z$ with values in $\mathcal{M}_{k} S^{0}\left(\mathbf{R}^{n} \times \mathbf{R}^{n}\right)$, from Taylor's formula, and (1.1.9) in [16]. We thus obtain $\mathcal{G}_{\left(z^{\prime}, z\right)}=\mathcal{G}_{\left(z^{\prime}, z\right)}^{I_{k}}+\Delta \tilde{\mathcal{G}}_{\left(z^{\prime}, z\right)}$, where the operator $\mathcal{G}_{\left(z^{\prime}, z\right)}^{I_{k}}$ is of the same form as $\mathcal{G}_{\left(z^{\prime}, z\right)}$ with the amplitude $g_{\left(z^{\prime}, z\right)}$ replaced by $I_{k}$. With the last point in Proposition 2.6, we have

$$
\left\|\tilde{\mathcal{G}}_{\left(z^{\prime}, z\right)}\right\|_{\left(\left(H^{(s)}\right)^{k},\left(H^{(s)}\right)^{k}\right)} \leq C,
$$

for $\Delta$ sufficiently small. Without any loss of generality we can thus assume that $g_{\left(z^{\prime}, z\right)}(x, \xi)=I_{k}$, i.e., $a_{0}(z, x, \xi)=0$.

Let $s \in \mathbf{R}$. Then the kernel of $\mathcal{B}_{\left(z^{\prime}, z\right)}:=\mathcal{G}_{\left(z^{\prime}, z\right)} \circ E^{(-s)}$ is given by

$$
B_{\left(z^{\prime}, z\right)}\left(x^{\prime}, x\right)=\int e^{i\left\langle x^{\prime}-x \mid \xi\right\rangle} e^{-\Delta a_{1}\left(z, x^{\prime}, \xi\right)}\langle\xi\rangle^{-s} d \xi
$$

The kernel of the adjoint operator of $\mathcal{B}_{\left(z^{\prime}, z\right)}$ is given by

$$
B_{\left(z^{\prime}, z\right)}^{*}\left(x^{\prime}, x\right)=\int e^{i\left\langle x^{\prime}-x \mid \xi\right\rangle} e^{-i \Delta b_{1}(z, x, \xi)-\Delta c_{1}(z, x, \xi)}\langle\xi\rangle^{-s} d \xi,
$$

since the matrix $b_{1}(z, x, \xi)$ and $c_{1}(z, x, \xi)$ are hermitian symmetric. Introducing $\mathcal{D}_{\left(z^{\prime}, z\right)}=\mathcal{B}_{\left(z^{\prime}, z\right)} \circ \mathcal{B}_{\left(z^{\prime}, z\right)}^{*}$, we find its kernel to be

$$
D_{\left(z^{\prime}, z\right)}\left(x^{\prime}, x\right)=\int e^{i\left\langle x^{\prime}-x \mid \xi\right\rangle} e^{-\Delta a_{1}\left(z, x^{\prime}, \xi\right)} e^{-i \Delta b_{1}(z, x, \xi)-\Delta c_{1}(z, x, \xi)}\langle\xi\rangle^{-2 s} d \xi
$$

which we write

$$
D_{\left(z^{\prime}, z\right)}\left(x^{\prime}, x\right)=\int e^{i\left\langle x^{\prime}-x \mid \xi\right\rangle}\left(w(z, .) e^{-\Delta d(z, .)} v(z, .)\right)\left(x^{\prime}, \xi\right)\left(w(z, .) e^{-\Delta \bar{d}(z, .)} v(z, .)\right)(x, \xi)\langle\xi\rangle^{-2 s} d \xi .
$$

With Taylor's formula we write

$$
v\left(z, x^{\prime}, \xi\right)=v(z, x, \xi)+\left\langle x^{\prime}-x \mid \tilde{v}\left(z, x^{\prime}, x, \xi\right)\right\rangle,
$$


with $\tilde{v}\left(z, x^{\prime}, x, \xi\right)$ bounded w.r.t. $z$ in $\left(\mathcal{M}_{k} S^{0}\left(X^{\prime} \times X \times \mathbf{R}^{n}\right)\right)^{n}$ by (1.1.9) in [16]. This yields

$$
D_{\left(z^{\prime}, z\right)}\left(x^{\prime}, x\right)=D_{\left(z^{\prime}, z\right), a}\left(x^{\prime}, x\right)+D_{\left(z^{\prime}, z\right), b}\left(x^{\prime}, x\right)+D_{\left(z^{\prime}, z\right), c}\left(x^{\prime}, x\right),
$$

where

$$
\begin{gathered}
D_{\left(z^{\prime}, z\right), a}\left(x^{\prime}, x\right)=\int e^{i\left\langle x^{\prime}-x \mid \xi\right\rangle} w\left(z, x^{\prime}, \xi\right) e^{-\Delta\left(d\left(z, x^{\prime}, \xi\right)+\bar{d}(z, x, \xi)\right)} v\left(z, x^{\prime}, \xi\right)\langle\xi\rangle^{-2 s} d \xi, \\
D_{\left(z^{\prime}, z\right), b}\left(x^{\prime}, x\right)=\int e^{i\left\langle x^{\prime}-x \mid \xi\right\rangle} w\left(z, x^{\prime}, \xi\right) e^{-\Delta d\left(z, x^{\prime}, \xi\right)}\left\langle x^{\prime}-x \mid \tilde{v}\left(z, x^{\prime}, x, \xi\right)\right\rangle w(z, x, \xi) e^{-\Delta \bar{d}(z, x, \xi)} v(z, x, \xi)\langle\xi\rangle^{-2 s} d \xi,
\end{gathered}
$$

and

$$
D_{\left(z^{\prime}, z\right), c}\left(x^{\prime}, x\right)=-\int e^{i\left\langle x^{\prime}-x \mid \xi\right\rangle} w\left(z, x^{\prime}, \xi\right) e^{-\Delta\left(d\left(z, x^{\prime}, \xi\right)+\bar{d}(z, x, \xi)\right)}\left\langle x^{\prime}-x \mid \tilde{v}\left(z, x^{\prime}, x, \xi\right)\right\rangle\langle\xi\rangle^{-2 s} d \xi
$$

We shall prove that the associated operators, namely $\mathcal{D}_{\left(z^{\prime}, z\right), a}, \mathcal{D}_{\left(z^{\prime}, z\right), b}$, and $\mathcal{D}_{\left(z^{\prime}, z\right), c}$, satisfy

$$
\left\|E^{(s)} \circ \mathcal{D}_{\left(z^{\prime}, z\right), a} \circ E^{(s)}\right\|_{\left(\left(L^{2}\right)^{k},\left(L^{2}\right)^{k}\right)} \leq 1+C \Delta, \quad\left\|E^{(s)} \circ\left(\mathcal{D}_{\left(z^{\prime}, z\right), b}+\mathcal{D}_{\left(z^{\prime}, z\right), c}\right) \circ E^{(s)}\right\|_{\left(\left(L^{2}\right)^{k},\left(L^{2}\right)^{k}\right)} \leq C \Delta,
$$

for some $C \geq 0$, uniformly in $z \in[0, Z]$ and $\Delta, \Delta$ sufficiently enough. It will then follow that

$$
\left\|\mathcal{G}_{\left(z^{\prime}, z\right)}\right\|_{\left(\left(H^{(s)}\right)^{k},\left(H^{(s)}\right)^{k}\right)}=\left\|\left(\mathcal{G}_{\left(z^{\prime}, z\right)}\right)^{*}\right\|_{\left(\left(H^{(s)}\right)^{k},\left(H^{(s)}\right)^{k}\right)} \leq \sqrt{1+C^{\prime} \Delta}, \quad C^{\prime} \geq 0,
$$

since $E^{(s)} \circ \mathcal{D}_{\left(z^{\prime}, z\right)} \circ E^{(s)}=\left(E^{(s)} \circ \mathcal{G}_{\left(z^{\prime}, z\right)} \circ E^{(-s)}\right) \circ\left(E^{(s)} \circ \mathcal{G}_{\left(z^{\prime}, z\right)} \circ E^{(-s)}\right)^{*}$, which will conclude the proof. We first estimate $\left\|E^{(s)} \circ \mathcal{D}_{\left(z^{\prime}, z\right), a} \circ E^{(s)}\right\|_{\left(\left(L^{2}\right)^{k},\left(L^{2}\right)^{k}\right)}$. The entry $i j$ of the matrix kernel $D_{\left(z^{\prime}, z\right), a}$ is given by

$$
\left(D_{\left(z^{\prime}, z\right), a}\right)_{i j}\left(x^{\prime}, x\right)=\sum_{1 \leq l \leq k} \int e^{i\left\langle x^{\prime}-x \mid \xi\right\rangle} e^{i \Delta\left(d_{b l l}\left(z, x^{\prime}, \xi\right)-d_{b, l}(z, x, \xi)\right)} \tilde{p}_{\Delta, l}\left(z, x^{\prime}, x, \xi\right) w_{i l}\left(z, x^{\prime}, \xi\right) v_{l j}\left(z, x^{\prime}, \xi\right)\langle\xi\rangle^{-2 s} d \xi,
$$

where we have set

$$
\tilde{p}_{\Delta, l}\left(z, x^{\prime}, x, \xi\right):=e^{-\Delta\left(d_{c, l}\left(z, x^{\prime}, \xi\right)+d_{c, l}(z, x, \xi)\right)},
$$

which satisfies Property $\left(Q_{L}\right)$ by Lemma 2.11, Remark 2.14 and Lemma 2.17.

For $l \in\{1, \ldots, k\}$, with Taylor's formula, we write

$$
d_{b, l}\left(z, x^{\prime}, \xi\right)-d_{b, l}(z, x, \xi)=\left\langle x^{\prime}-x \mid \tilde{d}_{b, l}\left(z, x^{\prime}, x, \xi\right)\right\rangle,
$$

where the function $\tilde{d}_{b, l}$ is smooth, homogeneous of degree one in $\xi,|\xi| \geq 1$, and continuous w.r.t. $z$ with values in $S^{1}\left(X^{\prime} \times X \times \mathbf{R}^{n}\right)$. We thus obtain that the change of variables $\xi \rightarrow \xi+\Delta \tilde{d}_{b, l}\left(z, x^{\prime}, x, \xi\right)=H_{l,\left(\Delta, z, x^{\prime}, x\right)}(\xi)$ is a global diffeomorphism for $\Delta$ small enough (uniformly in $z \in[0, Z]$ ). We denote $\tilde{\xi}_{l}\left(\Delta, z, x^{\prime}, x, \xi\right)=$ $H_{l,\left(\Delta, z, x^{\prime}, x\right)}^{-1}(\xi)$. We thus have

$$
\begin{aligned}
\left(D_{\left(z^{\prime}, z\right), a}\right)_{i j}\left(x^{\prime}, x\right)=\sum_{1 \leq l \leq k} \int e^{i\left\langle x^{\prime}-x \mid \xi\right\rangle} p_{\Delta, l}\left(z, x^{\prime}, x, \xi\right) w_{i l}\left(z, x^{\prime}, \tilde{\xi}_{l}\left(\Delta, z, x^{\prime}, x, \xi\right)\right) & \\
& \times v_{l j}\left(z, x^{\prime}, \tilde{\xi}_{l}\left(\Delta, z, x^{\prime}, x, \xi\right)\right)\left\langle\tilde{\xi}_{l}\left(\Delta, z, x^{\prime}, x, \xi\right)\right\rangle^{-2 s} \mathcal{J}_{l, \Delta}\left(z, x^{\prime}, x, \xi\right) d \xi,
\end{aligned}
$$

where $\mathcal{J}_{l, \Delta}\left(z, x^{\prime}, x, \xi\right)$ is the Jacobian and we have set

$$
p_{\Delta, l}\left(z, x^{\prime}, x, \xi\right):=\tilde{p}_{\Delta, l}\left(z, x^{\prime}, x, \tilde{\xi}_{l}\left(\Delta, z, x^{\prime}, x, \xi\right)\right) .
$$

For $\Delta$ sufficiently small, the function $\tilde{\xi}_{l}\left(\Delta, z, x^{\prime}, x, \xi\right)$ is homogeneous of degree one in $\xi$, continuous w.r.t. $z$, and $\mathscr{C}^{\infty}$ w.r.t. $\Delta$ with values in $S^{1}\left(X^{\prime} \times X \times \mathbf{R}^{n}\right)$ by Lemma 2.24 in [29]. By Lemma 1.7, $w_{i l}\left(z, x^{\prime}, \tilde{\xi}_{l}\left(\Delta, z, x^{\prime}, x, \xi\right)\right)$ and $v_{l j}\left(z, x^{\prime}, \tilde{\xi}_{l}\left(\Delta, z, x^{\prime}, x, \xi\right)\right)$ are $\mathscr{C}^{\infty}$ w.r.t. $\Delta$ and bounded w.r.t. $z$ with values in $S^{0}\left(X^{\prime} \times X \times \mathbf{R}^{n}\right)$, and $\left\langle\tilde{\xi}_{l}\left(\Delta, z, x^{\prime}, x, \xi\right)\right\rangle^{-2 s}$ is $\mathscr{C}^{\infty}$ w.r.t. $\Delta$ and bounded w.r.t. $z$ with values in $S^{-2 s}\left(X^{\prime} \times X \times \mathbf{R}^{n}\right)$ for $\Delta$ sufficiently small. The amplitude $p_{\Delta, l}\left(z, x^{\prime}, x, \xi\right)$ is bounded w.r.t. $z$ and $\Delta$ with values in $S_{\rho}^{0}\left(X^{\prime} \times X \times \mathbf{R}^{n}\right)$ and satisfies Property $\left(Q_{L}\right)$ by Lemma 2.13 for $\Delta$ sufficiently small. 
The Jacobian, $\mathcal{J}_{l, \Delta}\left(z, x^{\prime}, x, \xi\right)$, is homogeneous of degree zero in $\xi,|\xi| \geq 1$, and is continuous w.r.t. $z$ and $\mathscr{C}^{\infty}$ w.r.t. $\Delta$ with values in $S^{0}\left(\mathbf{R}^{2 n} \times \mathbf{R}^{n}\right)$ and $\left.\mathcal{J}_{l, \Delta}\left(z, x^{\prime}, x, \xi\right)\right|_{\Delta=0}=1$. With Taylor's formula and (1.1.9) in [16], we thus have

$$
\begin{aligned}
w_{i l}\left(z, x^{\prime}, \tilde{\xi}_{l}\left(\Delta, z, x^{\prime}, x, \xi\right)\right) v_{l j}\left(z, x^{\prime}, \tilde{\xi}_{l}\left(\Delta, z, x^{\prime}, x, \xi\right)\right)\left\langle\tilde{\xi}_{l}\left(\Delta, z, x^{\prime}, x, \xi\right)\right\rangle^{-2 s} & \\
& \times \mathcal{J}_{l, \Delta}\left(z, x^{\prime}, x, \xi\right)=w_{i l}\left(z, x^{\prime}, \xi\right) v_{l j}\left(z, x^{\prime}, \xi\right)\langle\xi\rangle^{-2 s}+\Delta k_{l i j, \Delta}\left(z, x^{\prime}, x, \xi\right),
\end{aligned}
$$

with $k_{l i j, \Delta}\left(z, x^{\prime}, x, \xi\right)$ bounded w.r.t. $z$ and $\Delta$ with values in $S^{-2 s}\left(X^{\prime} \times X \times \mathbf{R}^{n}\right)$. We thus obtain

$$
D_{\left(z^{\prime}, z\right), a}\left(x^{\prime}, x\right)=D_{\left(z^{\prime}, z\right), a}^{a}\left(x^{\prime}, x\right)+\Delta D_{\left(z^{\prime}, z\right), a}^{1}\left(x^{\prime}, x\right)
$$

where

$$
\left(D_{\left(z^{\prime}, z\right), a}^{1}\right)_{i j}\left(x^{\prime}, x\right)=\sum_{1 \leq l \leq k} \int e^{i\left\langle x^{\prime}-x \mid \xi\right\rangle}\left(p_{\Delta, l} k_{l i j, \Delta}\right)\left(z, x^{\prime}, x, \xi\right) d \xi
$$

and

$$
\left(D_{\left(z^{\prime}, z\right), a}^{a}\right)_{i j}\left(x^{\prime}, x\right)=\sum_{1 \leq l \leq k} \int e^{i\left\langle x^{\prime}-x \mid \xi\right\rangle} w_{i l}\left(z, x^{\prime}, \xi\right) p_{\Delta, l}\left(z, x^{\prime}, x, \xi\right) v_{l j}\left(z, x^{\prime}, \xi\right)\langle\xi\rangle^{-2 s} d \xi .
$$

Accordingly, we obtain $\mathcal{F}_{\left(z^{\prime}, z\right), a}:=E^{(s)} \circ \mathcal{D}_{\left(z^{\prime}, z\right), a} \circ E^{(s)}=\mathcal{F}_{\left(z^{\prime}, z\right), a}^{a}+\mathcal{F}_{\left(z^{\prime}, z\right), a}^{1}$. From the boundedness of the amplitudes w.r.t. $z$ and $\Delta$ stated above, we obtain $\left\|\mathcal{F}_{\left(z^{\prime}, z\right), a}^{1}\right\|_{\left(\left(L^{2}\right)^{k},\left(L^{2}\right)^{k}\right)} \leq K^{(1)}$, uniformly in $z \in[0, Z]$ and $\Delta, \Delta$ sufficiently enough, by the Calderón-Vaillancourt Theorem (see [23, Chapter 7, Sections 1,2] or [39, Section XIII-2]).

Observe now that

$$
D_{\left(z^{\prime}, z\right), a}^{a}=\int e^{i\left\langle x^{\prime}-x \mid \xi\right\rangle} w\left(z, x^{\prime}, \xi\right) p_{\Delta}\left(z, x^{\prime}, x, \xi\right) v\left(z, x^{\prime}, \xi\right)\langle\xi\rangle^{-2 s} d \xi,
$$

where $p_{\Delta}\left(z, x^{\prime}, x, \xi\right)$ is the diagonal matrix with $p_{\Delta, l}\left(z, x^{\prime}, x, \xi\right)$ for diagonal entries. Setting,

$$
\mu_{\Delta}\left(z, x^{\prime}, x, \xi\right)=w\left(z, x^{\prime}, \xi\right) p_{\Delta}\left(z, x^{\prime}, x, \xi\right) v\left(z, x^{\prime}, \xi\right),
$$

the operator $\mathcal{F}_{\left(z^{\prime}, z\right), a}^{a}$ is pseudodifferential and has for symbol

$$
\left(\langle\xi\rangle^{s} \# \sigma\left\{\mu_{\Delta}\left(z, x^{\prime}, x, \xi\right)\langle\xi\rangle^{-2 s}\right\} \#\langle\xi\rangle^{s}\right)\left(x^{\prime}, \xi\right) .
$$

The matrix amplitude $\mu_{\Delta}\left(z, x^{\prime}, x, \xi\right)$ is bounded w.r.t. $\Delta$ and $z$ with values in $S_{\rho}^{0}\left(X^{\prime} \times X \times \mathbf{R}^{n}\right)$ and satisfies Property $\left(Q_{L}\right)$ by Proposition 2.15 . By Lemma 2.18 we see that

$$
\sigma\left\{\mu_{\Delta}\left(z, x^{\prime}, x, \xi\right)\langle\xi\rangle^{-2 s}\right\}-\mu_{\Delta}\left(z, x^{\prime}, x^{\prime}, \xi\right)\langle\xi\rangle^{-2 s}=\Delta \lambda_{\Delta}\left(z, x^{\prime}, \xi\right)
$$

where $\lambda_{\Delta}$ is bounded w.r.t. $\Delta$ and $z$ with values in $\mathcal{M}_{k} S_{\rho}^{-2 s}\left(\mathbf{R}^{n} \times \mathbf{R}^{n}\right)$. We thus obtain

$$
\mathcal{F}_{\left(z^{\prime}, z\right), a}^{a}=\mathcal{F}_{\left(z^{\prime}, z\right), a}^{b}+\Delta \mathcal{F}_{\left(z^{\prime}, z\right), a}^{2},
$$

where $\mathcal{F}_{\left(z^{\prime}, z\right), a}^{b}$ has for symbol

$$
f_{\left(z^{\prime}, z\right), a}^{b}\left(z, x^{\prime}, \xi\right):=\left(\langle\xi\rangle^{s} \#\left(\mu_{\Delta}\left(z, x^{\prime}, x^{\prime}, \xi\right)\langle\xi\rangle^{-2 s}\right) \#\langle\xi\rangle^{s}\right)\left(x^{\prime}, \xi\right)=\left(\langle\xi\rangle^{s} \# \mu_{\Delta}\left(z, x^{\prime}, x^{\prime}, \xi\right)\langle\xi\rangle^{-s}\right)\left(x^{\prime}, \xi\right)
$$

and $\left\|\mathcal{F}_{\left(z^{\prime}, z\right), a}^{2}\right\|_{\left(\left(L^{2}\right)^{k},\left(L^{2}\right)^{k}\right)} \leq K^{(2)}$, uniformly in $z \in[0, Z]$ and $\Delta, \Delta$ sufficiently small, invoking the same theorems as above.

The proof of the following lemma can be adapted to the matrix case from the proof of Lemma 2.25 in [29].

Lemma 2.23.

$$
\left(\langle\xi\rangle^{s} \#\left(\mu_{\Delta}(z, x, x, \xi)\langle\xi\rangle^{-s}\right)\right)(z, x, \xi)-\mu_{\Delta}(z, x, x, \xi)=\Delta v_{\Delta}(z, x, \xi)
$$

where $v_{\Delta}(z, x, \xi)$ is bounded w.r.t. $z$ and $\Delta$ with values in $\mathcal{M}_{k} S_{\rho}^{0}\left(X \times \mathbf{R}^{n}\right)$. 
With this lemma we obtain

$$
\mathcal{F}_{\left(z^{\prime}, z\right), a}^{b}=\mathcal{F}_{\left(z^{\prime}, z\right), a}^{c}+\Delta \mathcal{F}_{\left(z^{\prime}, z\right), a}^{3},
$$

where $\mathcal{F}_{\left(z^{\prime}, z\right), a}^{c}$ has for symbol $\mu_{\Delta}\left(z, x^{\prime}, x^{\prime}, \xi\right)$ and $\left\|\mathcal{F}_{\left(z^{\prime}, z\right), a}^{3}\right\|_{\left(\left(L^{2}\right)^{k},\left(L^{2}\right)^{k}\right)} \leq K^{(3)}$ uniformly in $z \in[0, Z]$ and $\Delta, \Delta$ small enough. With Theorem 2.21, we obtain $\left\|\mathcal{F}_{\left(z^{\prime}, z\right), a}^{c}\right\|_{\left(\left(L^{2}\right)^{k},\left(L^{2}\right)^{k}\right)} \leq 1+K^{(c)} \Delta$, for $K^{(c)} \geq 0$, and we thus have the desired estimate, i.e.,

$$
\left\|\mathcal{F}_{\left(z^{\prime}, z\right), a}\right\|_{\left(\left(L^{2}\right)^{k},\left(L^{2}\right)^{k}\right)} \leq 1+\left(K^{(c)}+K^{(1)}+K^{(2)}+K^{(3)}\right) \Delta,
$$

uniformly in $z \in[0, Z]$ and $\Delta, \Delta$ small enough.

We now turn to estimating the operator norm $\left\|\mathcal{D}_{\left(z^{\prime}, z\right), b}+\mathcal{D}_{\left(z^{\prime}, z\right), c}\right\|_{\left(\left(L^{2}\right)^{k},\left(L^{2}\right)^{k}\right)}$. We observe that $\left(x_{j}^{\prime}-x_{j}\right) e^{i\left\langle x^{\prime}-x \mid \xi\right\rangle}=$ $-i \partial_{\xi_{j}} e^{i\left\langle x^{\prime}-x \mid \xi\right\rangle}$. By integration by parts we obtain

$$
D_{\left(z^{\prime}, z\right), b}\left(x^{\prime}, x\right)=i \int e^{i\left\langle x^{\prime}-x \mid \xi\right\rangle}\left\langle\nabla_{\xi} \mid w\left(z, x^{\prime}, \xi\right) e^{i \Delta d\left(z, x^{\prime}, \xi\right)} \tilde{v}\left(z, x^{\prime}, x, \xi\right) w(z, x, \xi) e^{-i \Delta d(z, x, \xi)} v(z, x, \xi)\langle\xi\rangle^{-2 s}\right\rangle d \xi
$$

which, by Leibniz formula, we write

$$
D_{\left(z^{\prime}, z\right), b}\left(x^{\prime}, x\right)=D_{\left(z^{\prime}, z\right), b}^{a}\left(x^{\prime}, x\right)+\Delta D_{\left(z^{\prime}, z\right), b}^{1}\left(x^{\prime}, x\right)
$$

where

$$
D_{\left(z^{\prime}, z\right), b}^{a}\left(x^{\prime}, x\right)=i \int e^{i\left\langle x^{\prime}-x \mid \xi\right\rangle} \alpha_{\left(z^{\prime}, z\right), b}^{a}\left(x^{\prime}, x, \xi\right) d \xi, \quad D_{\left(z^{\prime}, z\right), b}^{1}\left(x^{\prime}, x\right)=i \int e^{i\left\langle x^{\prime}-x \mid \xi\right\rangle} \alpha_{\left(z^{\prime}, z\right), b}^{1}\left(x^{\prime}, x, \xi\right) d \xi,
$$

with

$$
\begin{aligned}
\alpha_{\left(z^{\prime}, z\right), b}^{a}\left(x^{\prime}, x, \xi\right) & =\left\langle\nabla_{\xi} w\left(z, x^{\prime}, \xi\right) \mid e^{-\Delta d\left(z, x^{\prime}, \xi\right)} \tilde{v}\left(z, x^{\prime}, x, \xi\right) w(z, x, \xi) e^{-\Delta \bar{d}(z, x, \xi)} v(z, x, \xi)\langle\xi\rangle^{-2 s}\right\rangle \\
& +w\left(z, x^{\prime}, \xi\right) e^{-\Delta d\left(z, x^{\prime}, \xi\right)}\left\langle\nabla_{\xi} \mid \tilde{v}\left(z, x^{\prime}, x, \xi\right) w(z, x, \xi)\right\rangle e^{-\Delta \bar{d}(z, x, \xi)} v(z, x, \xi)\langle\xi\rangle^{-2 s} \\
& +w\left(z, x^{\prime}, \xi\right) e^{-\Delta d\left(z, x^{\prime}, \xi\right)}\left\langle\tilde{v}\left(z, x^{\prime}, x, \xi\right) w(z, x, \xi) e^{-\Delta \bar{d}(z, x, \xi)} \mid \nabla_{\xi}\left(v(z, x, \xi)\langle\xi\rangle^{-2 s}\right)\right\rangle
\end{aligned}
$$

and

$$
\begin{aligned}
\alpha_{\left(z^{\prime}, z\right), b}^{1}\left(x^{\prime}, x, \xi\right) & =-w\left(z, x^{\prime}, \xi\right)\left\langle\nabla_{\xi} d\left(z, x^{\prime}, \xi\right) e^{-\Delta d\left(z, x^{\prime}, \xi\right)} \mid \tilde{v}\left(z, x^{\prime}, x, \xi\right)\right\rangle w(z, x, \xi) e^{-\Delta \bar{d}(z, x, \xi)} v(z, x, \xi)\langle\xi\rangle^{-2 s} \\
& -w\left(z, x^{\prime}, \xi\right) e^{-\Delta d\left(z, x^{\prime}, \xi\right)}\left\langle\tilde{v}\left(z, x^{\prime}, x, \xi\right) w(z, x, \xi) \mid \nabla_{\xi} \bar{d}(z, x, \xi)\right\rangle e^{-\Delta \bar{d}(z, x, \xi)} v(z, x, \xi)\langle\xi\rangle^{-2 s}
\end{aligned}
$$

Note that neither $\alpha_{\left(z^{\prime}, z\right), b}^{a}$ or $\alpha_{\left(z^{\prime}, z\right), b}^{1}$ are amplitudes in general. However, we observe that all the kernel components $\left(D_{\left(z^{\prime}, z\right), b}^{a}\right)_{i j}\left(x^{\prime}, x\right)$ and $\left(D_{\left(z^{\prime}, z\right), b}^{1}\right)_{i j}\left(x^{\prime}, x\right), 1 \leq i, j \leq k$, are of the form of the scalar kernel $A_{\left(z^{\prime}, z\right)}\left(x^{\prime}, x\right)$ in (1.2) in Section 1, with an amplitude bounded w.r.t. $z$ and $\Delta$ with values in $S_{\rho}^{-2 s-1}\left(X^{\prime} \times X \times \mathbf{R}^{n}\right)$ and $S_{\rho}^{-2 s}\left(X^{\prime} \times X \times \mathbf{R}^{n}\right)$ respectively. If $\mathcal{D}_{\left(z^{\prime}, z\right), b}^{1}$ is the operator associated to the kernel $D_{\left(z^{\prime}, z\right), b}^{1}\left(x^{\prime}, x\right)$, for all $r \in \mathbf{R}$, there exists $L^{(1)} \geq 0$ such that

$$
\left\|\mathcal{D}_{\left(z^{\prime}, z\right), b}^{1}\right\|_{\left(\left(H^{(r)}\right)^{k},\left(H^{(r+2 s)}\right)^{k}\right)} \leq L^{(1)},
$$

by Theorem 1.12 , uniformly in $z \in[0, Z]$ and $\Delta, \Delta$ sufficiently small.

In the three terms in the expression of $\alpha_{\left(z^{\prime}, z\right), b}^{a}\left(x^{\prime}, x, \xi\right)$, we write

$$
e^{-\Delta d\left(z, x^{\prime}, \xi\right)}=1-\Delta d\left(z, x^{\prime}, \xi\right) \int_{0}^{1} e^{-\sigma \Delta d\left(z, x^{\prime}, \xi\right)} d \sigma .
$$

We thus obtain

$$
D_{\left(z^{\prime}, z\right), b}^{a}\left(x^{\prime}, x\right)=D_{\left(z^{\prime}, z\right), b}^{b}\left(x^{\prime}, x\right)+\Delta D_{\left(z^{\prime}, z\right), b}^{2}\left(x^{\prime}, x\right)
$$


where

$$
D_{\left(z^{\prime}, z\right), b}^{b}\left(x^{\prime}, x\right)=i \int e^{i\left\langle x^{\prime}-x \mid \xi\right\rangle} \alpha_{\left(z^{\prime}, z\right), b}^{b}\left(x^{\prime}, x, \xi\right) d \xi
$$

with

$$
\begin{aligned}
\alpha_{\left(z^{\prime}, z\right), b}^{b}\left(x^{\prime}, x, \xi\right) & =\left\langle\nabla_{\xi} w\left(z, x^{\prime}, \xi\right) \mid \tilde{v}\left(z, x^{\prime}, x, \xi\right)\right\rangle w(z, x, \xi) e^{-\Delta \bar{d}(z, x, \xi)} v(z, x, \xi)\langle\xi\rangle^{-2 s} \\
& +w\left(z, x^{\prime}, \xi\right)\left\langle\nabla_{\xi} \mid \tilde{v}\left(z, x^{\prime}, x, \xi\right) w(z, x, \xi)\right\rangle e^{-\Delta \bar{d}(z, x, \xi)} v(z, x, \xi)\langle\xi\rangle^{-2 s} \\
& +w\left(z, x^{\prime}, \xi\right)\left\langle\tilde{v}\left(z, x^{\prime}, x, \xi\right) w(z, x, \xi) e^{-\Delta \bar{d}(z, x, \xi)} \mid \nabla_{\xi}\left(v(z, x, \xi)\langle\xi\rangle^{-2 s}\right)\right\rangle,
\end{aligned}
$$

and

$$
D_{\left(z^{\prime}, z\right), b}^{2}\left(x^{\prime}, x\right)=i \int_{0}^{1} \int^{i\left\langle x^{\prime}-x \mid \xi\right\rangle} \alpha_{\left(z^{\prime}, z\right), b}^{2}\left(\sigma, x^{\prime}, x, \xi\right) d \xi d \sigma
$$

with

$$
\begin{aligned}
\alpha_{\left(z^{\prime}, z\right), b}^{2}\left(\sigma, x^{\prime}, x, \xi\right) & =-\left\langle\nabla_{\xi} w\left(z, x^{\prime}, \xi\right) \mid d\left(z, x^{\prime}, \xi\right) e^{-\sigma \Delta d\left(z, x^{\prime}, \xi\right)} \tilde{v}\left(z, x^{\prime}, x, \xi\right) w(z, x, \xi) e^{-\Delta \bar{d}(z, x, \xi)} v(z, x, \xi)\langle\xi\rangle^{-2 s}\right\rangle \\
& -w\left(z, x^{\prime}, \xi\right) d\left(z, x^{\prime}, \xi\right) e^{-\sigma \Delta d\left(z, x^{\prime}, \xi\right)}\left\langle\nabla_{\xi} \mid \tilde{v}\left(z, x^{\prime}, x, \xi\right) w(z, x, \xi)\right\rangle e^{-\Delta \bar{d}(z, x, \xi)} v(z, x, \xi)\langle\xi\rangle^{-2 s} \\
& -w\left(z, x^{\prime}, \xi\right) d\left(z, x^{\prime}, \xi\right) e^{-\sigma \Delta d\left(z, x^{\prime}, \xi\right)}\left\langle\tilde{v}\left(z, x^{\prime}, x, \xi\right) w(z, x, \xi) e^{-\Delta \bar{d}(z, x, \xi)} \mid \nabla_{\xi}\left(v(z, x, \xi)\langle\xi\rangle^{-2 s}\right)\right\rangle .
\end{aligned}
$$

The kernel components $\left(D_{\left(z^{\prime}, z\right), b}^{2}\right)_{i j}\left(x^{\prime}, x\right), 1 \leq i, j \leq k$, are of the form of the scalar kernel $A_{\left(z^{\prime}, z\right)}\left(x^{\prime}, x\right)$ in (1.2) in Section 1, with an amplitude bounded w.r.t. $z$ and $\Delta$ with values and $S_{\rho}^{-2 s}\left(X^{\prime} \times X \times \mathbf{R}^{n}\right)$. Hence, for all $r \in \mathbf{R}$, there exists $L^{(2)} \geq 0$ such that

$$
\left\|\mathcal{D}_{\left(z^{\prime}, z\right), b}^{2}\right\|_{\left(\left(H^{(r)}\right)^{k},\left(H^{(r+2 s)}\right)^{k}\right)} \leq L^{(2)},
$$

uniformly in $z \in[0, Z]$ and $\Delta, \Delta$ sufficiently enough, for $\mathcal{D}_{\left(z^{\prime}, z\right), b}^{2}$, the operator associated to the kernel $D_{\left(z^{\prime}, z\right), b}^{2}\left(x^{\prime}, x\right)$, by Theorem 1.12 .

We proceed similarly with the exponential term left in the expression of the three terms that compose $\alpha_{\left(z^{\prime}, z\right), b}^{b}\left(x^{\prime}, x, \xi\right)$ above, writing

$$
e^{-\Delta \bar{d}\left(z, x^{\prime}, \xi\right)}=1-\Delta \bar{d}\left(z, x^{\prime}, \xi\right) \int_{0}^{1} e^{-\sigma \Delta \bar{d}\left(z, x^{\prime}, \xi\right)} d \sigma .
$$

and we obtain

$$
D_{\left(z^{\prime}, z\right), b}^{b}\left(x^{\prime}, x\right)=D_{\left(z^{\prime}, z\right), b}^{c}\left(x^{\prime}, x\right)+\Delta D_{\left(z^{\prime}, z\right), b}^{3}\left(x^{\prime}, x\right)
$$

where

$$
D_{\left(z^{\prime}, z\right), b}^{c}\left(x^{\prime}, x\right)=i \int e^{i\left\langle x^{\prime}-x \mid \xi\right\rangle} \alpha_{\left(z^{\prime}, z\right), b}^{c}\left(x^{\prime}, x, \xi\right) d \xi
$$

with

$$
\begin{aligned}
\alpha_{\left(z^{\prime}, z\right), b}^{c}\left(x^{\prime}, x, \xi\right) & =\left\langle\nabla_{\xi} w\left(z, x^{\prime}, \xi\right) \mid \tilde{v}\left(z, x^{\prime}, x, \xi\right)\right\rangle w(z, x, \xi) v(z, x, \xi)\langle\xi\rangle^{-2 s} \\
& +w\left(z, x^{\prime}, \xi\right)\left\langle\nabla_{\xi} \mid \tilde{v}\left(z, x^{\prime}, x, \xi\right) w(z, x, \xi)\right\rangle v(z, x, \xi)\langle\xi\rangle^{-2 s} \\
& +w\left(z, x^{\prime}, \xi\right)\left\langle\tilde{v}\left(z, x^{\prime}, x, \xi\right) w(z, x, \xi) \mid \nabla_{\xi}\left(v(z, x, \xi)\langle\xi\rangle^{-2 s}\right)\right\rangle,
\end{aligned}
$$

and for all $r \in \mathbf{R}$, there exists $L^{(3)} \geq 0$ such that

$$
\left\|\mathcal{D}_{\left(z^{\prime}, z\right), b}^{3}\right\|_{\left(\left(H^{(r)}\right)^{k},\left(H^{(r+2 s)}\right)^{k}\right)} \leq L^{(3)}
$$


uniformly in $z \in[0, Z]$ and $\Delta, \Delta$ sufficiently enough, for $\mathcal{D}_{\left(z^{\prime}, z\right), b}^{3}$, the operator associated to the kernel $D_{\left(z^{\prime}, z\right), b}^{3}\left(x^{\prime}, x\right)$, with the same argumentation as above.

We observe

$$
\alpha_{\left(z^{\prime}, z\right), b}^{c}\left(x^{\prime}, x, \xi\right)=\left\langle\nabla_{\xi} \mid w\left(z, x^{\prime}, \xi\right) \tilde{v}\left(z, x^{\prime}, x, \xi\right) w(z, x, \xi) v(z, x, \xi)\langle\xi\rangle^{-2 s}\right\rangle=\left\langle\nabla_{\xi} \mid w\left(z, x^{\prime}, \xi\right) \tilde{v}\left(z, x^{\prime}, x, \xi\right)\langle\xi\rangle^{-2 s}\right\rangle
$$

recalling that $v(z, x, \xi)=(w(z, x, \xi))^{-1}$. An integration by parts yields

$$
D_{\left(z^{\prime}, z\right), b}^{c}\left(x^{\prime}, x\right)=\int e^{i\left\langle x^{\prime}-x \mid \xi\right\rangle} w\left(z, x^{\prime}, \xi\right)\left\langle x^{\prime}-x \mid \tilde{v}\left(z, x^{\prime}, x, \xi\right)\right\rangle\langle\xi\rangle^{-2 s} d \xi,
$$

and thus we obtain

$$
D_{\left(z^{\prime}, z\right), b}^{c}\left(x^{\prime}, x\right)=\int e^{i\left\langle x^{\prime}-x \mid \xi\right\rangle} w\left(z, x^{\prime}, \xi\right)\left(v\left(z, x^{\prime}, \xi\right)-v(z, x, \xi)\right)\langle\xi\rangle^{-2 s} d \xi,
$$

since $v\left(z, x^{\prime}, \xi\right)=v(z, x, \xi)+\left\langle x^{\prime}-x \mid \tilde{v}\left(z, x^{\prime}, x, \xi\right)\right\rangle$. We have thus obtained

$$
D_{\left(z^{\prime}, z\right), b}\left(x^{\prime}, x\right)=D_{\left(z^{\prime}, z\right), b}^{c}\left(x^{\prime}, x\right)+\Delta\left(D_{\left(z^{\prime}, z\right), b}^{1}\left(x^{\prime}, x\right)+D_{\left(z^{\prime}, z\right), b}^{2}\left(x^{\prime}, x\right)+D_{\left(z^{\prime}, z\right), b}^{3}\left(x^{\prime}, x\right)\right),
$$

with

$$
\left\|\mathcal{D}_{\left(z^{\prime}, z\right), b}^{1}+\mathcal{D}_{\left(z^{\prime}, z\right), b}^{2}+\mathcal{D}_{\left(z^{\prime}, z\right), b}^{3}\right\|_{\left(\left(H^{(r)}\right)^{k},\left(H^{(r+2 s)}\right)^{k}\right)} \leq L^{(1)}+L^{(2)}+L^{(3)},
$$

uniformly in $z \in[0, Z]$ and $\Delta, \Delta$ sufficiently small.

In a similar fashion, we obtain

$$
D_{\left(z^{\prime}, z\right), c}\left(x^{\prime}, x\right)=-D_{\left(z^{\prime}, z\right), b}^{c}\left(x^{\prime}, x\right)+\Delta D_{\left(z^{\prime}, z\right), c}^{1}\left(x^{\prime}, x\right),
$$

with, for the operator $\mathcal{D}_{\left(z^{\prime}, z\right), c}^{1}$ associated to the kernel $D_{\left(z^{\prime}, z\right), c}^{1}\left(x^{\prime}, x\right)$,

$$
\left\|\mathcal{D}_{\left(z^{\prime}, z\right), c}^{1}\right\|_{\left(\left(H^{(r)}\right)^{k},\left(H^{(r+2 s)}\right)^{k}\right)} \leq M^{(1)},
$$

for $M^{(1)} \geq 0$, uniformly in $z \in[0, Z]$ and $\Delta, \Delta$ sufficiently small. With $r=-s$ we thus obtain the expected estimate

$$
\left\|E^{(s)} \circ\left(\mathcal{D}_{\left(z^{\prime}, z\right), b}+\mathcal{D}_{\left(z^{\prime}, z\right), c}\right) \circ E^{(s)}\right\|_{\left(\left(L^{2}\right)^{k},\left(L^{2}\right)^{k}\right)} \leq C \Delta,
$$

uniformly in $z \in[0, Z]$ and $\Delta, \Delta$ sufficiently small.

\section{Convergence properties of the Ansatz $\mathcal{W}_{\mathfrak{p}, z}$ in the symmetric case}

As in Section 2, the $z$-family of symbols $a_{1}\left(z\right.$,.) satisfies Assumptions 2.1 and 2.2. Let $\rho \in\left[\frac{1}{2}, 1\right]$. We assume that $c_{1}$ is chosen such that

$$
p_{\Delta}(z, .):=e^{-\Delta c_{1}(z, .)}
$$

takes values in $\mathcal{M}_{k} S_{\rho}^{0}\left(X \times \mathbf{R}^{n}\right)$ (see Lemma 2.11 and corollary 2.16).

We first define the Ansatz that approximates the solution operator to (2.1)-(2.2). The regularity properties of the thin-slab propagator $\mathcal{G}_{\left(z^{\prime}, z\right)}$ given in Proposition 2.6 allow to compose operators of the form of $\mathcal{G}_{\left(z^{\prime}, z\right)}$.

Definition 3.1. Let $\mathfrak{P}=\left\{z^{(0)}, z^{(1)}, \ldots, z^{(N)}\right\}$ be a subdivision of $[0, Z]$ with $0=z^{(0)}<z^{(1)}<\cdots<z^{(N)}=Z$. We set $\Delta_{\mathfrak{B}}=\sup _{i=1, \ldots, N}\left(z^{(i)}-z^{(i-1)}\right)$. The operator $\mathcal{W}_{\Re, z}$ is defined as

$$
\mathcal{W}_{\mathfrak{P}, z}:= \begin{cases}\mathcal{G}_{(z, 0)} & \text { if } 0 \leq z \leq z^{(1)}, \\ \mathcal{G}_{\left(z, z^{(k)}\right)} \prod_{i=k}^{1} \mathcal{G}_{\left(z^{(i)}, z^{(i-1)}\right)} & \text { if } z^{(k)} \leq z \leq z^{(k+1)} .\end{cases}
$$

Thanks to the estimate proven in Theorem 2.22 we can now obtain the following proposition. 
Proposition 3.2. Let $s \in \mathbf{R}$. There exists $K \geq 0$ such that for every subdivision $\mathfrak{P}$ of $[0, Z]$ we have

$$
\forall z \in[0, Z],\left\|\mathcal{W}_{\mathfrak{P}, z}\right\|_{\left(\left(H^{(s)}\right)^{k},\left(H^{(s)}\right)^{k}\right)} \leq K,
$$

for $\Delta_{\mathfrak{P}}$ sufficiently small.

Proof. By Theorem 2.22, there exits $M \geq 0$ such that if $\Delta=z^{\prime}-z$ is small enough then we have $\left\|\mathcal{G}_{\left(z^{\prime}, z\right)}\right\|_{\left(\left(H^{(s)}\right)^{k},\left(H^{(s)}\right)^{k}\right)} \leq 1+\Delta M$ for all $z \in[0, Z]$. For $\Delta_{\mathfrak{P}}$ sufficiently small we then obtain

$$
\left\|\mathcal{W}_{\mathfrak{P}, z}\right\|_{\left(\left(H^{(s)}\right)^{k},\left(H^{(s)}\right)^{k}\right)} \leq \prod_{i=0}^{N-1}\left(1+M\left(z^{(i+1)}-z^{(i)}\right)\right) \leq \prod_{i=0}^{N-1} e^{M\left(z^{(i+1)}-z^{(i)}\right)}=e^{M Z} .
$$

We have the following regularity result for the Ansatz $\mathcal{W}_{\mathfrak{P}, z}$, by adapting the proof of Proposition 3.5 in [29] to the present matrix case.

Proposition 3.3. Let $s \in \mathbf{R}, \mathfrak{P}$ a subdivision of $[0, Z]$ as in Definition 3.1 and let $u_{0} \in\left(H^{(s+1)}(X)\right)^{k}$. Then the map $z \mapsto \mathcal{W}_{\mathfrak{P}, z}\left(u_{0}\right)$ is in $\mathscr{C}^{0}\left([0, Z],\left(H^{(s+1)}(X)\right)^{k}\right)$ and is piecewise $\mathscr{C}^{1}\left([0, Z],\left(H^{(s)}(X)\right)^{k}\right)$ if $\mathfrak{P}$ is chosen such that $\Delta_{\mathfrak{P}}$ is small enough. The map $z \mapsto \mathcal{W}_{\mathfrak{P}, z}\left(u_{0}\right)$ is in fact globally Lipschitz with $C \geq 0$ such that

$$
\left\|\mathcal{W}_{\mathfrak{P}, z^{\prime}}\left(u_{0}\right)-\mathcal{W}_{\mathfrak{P}, z}\left(u_{0}\right)\right\|_{\left(H^{(s)}\right)^{k}} \leq C\left|z^{\prime}-z\right|\left\|u_{0}\right\|_{\left(H^{(s+1)}\right)^{k}},
$$

where the constant $C$ is uniform w.r.t. $\mathfrak{P}, z^{\prime}$ and $z$, if $\Delta_{\mathfrak{P}}$ is sufficiently small.

Before proceeding to estimating the approximation error made between the Ansatz $W_{\mathfrak{P}, z}$ and the solution operator $U(z, 0)$ of $(2.1)-(2.2)$, we need to establish a $\psi$ DO-FIO composition formula adapted to the case of matrix phase functions such as $\phi_{\left(z^{\prime}, z\right)}$ given in (2.5).

\subsection{A composition formula in the case of matrix phases}

Composition formulae like those of Proposition 1.10 do not extend straightforwardly in general to the case of matrix phases. We can however obtain a close-enough result which will be of use in the sequel.

Theorem 3.4. let $\rho^{\prime}, \rho^{\prime \prime} \in\left[\frac{1}{2}, 1\right]$. Let $\mu(z, x, \xi)$ be bounded w.r.t. $z$ with values in $\mathcal{M}_{k} S_{\rho^{\prime}}^{m}\left(X \times \mathbf{R}^{n}\right)$, and the operator $\mathcal{A}_{\left(z^{\prime}, z\right)}$ defined by

$$
\mathcal{A}_{\left(z^{\prime}, z\right)}(u)\left(x^{\prime}\right)=\iint e^{i\left\langle x^{\prime}-x \mid \xi\right\rangle} \sigma_{\left(z^{\prime}, z\right)}\left(x^{\prime}, \xi\right) e^{-\Delta a_{1}\left(z, x^{\prime}, x, \xi\right)} u(x) d \xi d x,
$$

where $0 \leq z \leq z^{\prime} \leq Z, \Delta=z^{\prime}-z$, with $\sigma_{\left(z^{\prime}, z\right)}\left(x^{\prime}, \xi\right)$ bounded w.r.t. $z^{\prime}$ and $z$ with values in $\mathcal{M}_{k} S_{\rho^{\prime \prime}}^{m^{\prime}}\left(X \times \mathbf{R}^{n}\right)$. Then, for $\Delta$ sufficiently small, we have

$$
\mu\left(z, x, D_{x}\right) \circ \mathcal{A}_{\left(z^{\prime}, z\right)}=\mathcal{B}_{\left(z^{\prime}, z\right)}+\Delta \mathcal{R}_{\left(z^{\prime}, z\right)},
$$

where for all $s \in \mathbf{R}$ there exists $C \geq 0$ such that

$$
\left\|\mathcal{R}_{\left(z^{\prime}, z\right)}\right\|_{\left(\left(H^{(s)}\right)^{k},\left(H^{\left(s-m-m^{\prime}\right)}\right)^{k}\right)} \leq C, \quad 0 \leq z \leq z^{\prime} \leq Z,
$$

and the operator $\mathcal{B}_{\left(z^{\prime}, z\right)}$ has for kernel

$$
B_{\left(z^{\prime}, z\right)}\left(x^{\prime}, x\right)=\int e^{i\left\langle x^{\prime}-x \mid \xi\right\rangle} q_{\left(z^{\prime}, z\right)}\left(x^{\prime}, \xi\right) e^{i \Delta b_{1}\left(z, x^{\prime}, \xi\right)} d \xi
$$

with $q_{\left(z^{\prime}, z\right)}\left(x^{\prime}, \xi\right)$ bounded w.r.t. to $z^{\prime}$ and $z$ with values in $\mathcal{M}_{k} S_{\min \left(\rho, \rho^{\prime}, \rho^{\prime \prime}\right)}^{m+m^{\prime}}\left(X \times \mathbf{R}^{n}\right)$ and given by the oscillatory integral representation

(3.3) $q_{\left(z^{\prime}, z\right)}\left(x^{\prime}, \xi\right)=\iint e^{i\left\langle x^{\prime}-y \mid \eta-\xi\right\rangle} \mu\left(z, x^{\prime}, \eta\right) \sigma_{\left(z^{\prime}, z\right)}(y, \xi) p_{\Delta}(z, y, \xi) w(z, y, \xi) e^{i \Delta\left(d_{b}(z, y, \xi)-d_{b}\left(z, x^{\prime}, \xi\right)\right)} v(z, y, \xi) d \eta d y$, with $p_{\Delta}(z, y, \xi)$ given in (3.1).

The proof of Theorem 3.4 can be found in Appendix A. 


\subsection{Error estimation, convergence}

To estimate the norm of $\mathcal{W}_{\Re, z}\left(u_{0}\right)-U(z, 0)\left(u_{0}\right)$ in some Sobolev space, where $U(z, 0)$ is the solution operator of (2.1)-(2.2), we first need to have an understanding of the infinitesimal error made by the use of the thinslab propagator, i.e., find a bound for

$$
\left(\partial_{z^{\prime}}+a\left(z^{\prime}, x, D_{x}\right)\right) \circ \mathcal{G}_{\left(z^{\prime}, z\right)}(u), \quad 0 \leq z \leq z^{\prime} \leq Z, u \in\left(H^{(s)}\right)^{k},
$$

in some properly chosen norm when $\Delta=z^{\prime}-z$ is small. For the next proposition we shall need the following assumption.

Assumption 3.5. The matrix symbol $a(z,$.$) is in \mathscr{C}^{0, \alpha}\left([0, Z], \mathcal{M}_{k} S^{1}\left(X \times \mathbf{R}^{n}\right)\right), 0<\alpha \leq 1$, i.e., Hölder continuous w.r.t. $z$ with values in $\mathcal{M}_{k} S^{1}\left(X \times \mathbf{R}^{n}\right)$, in the sense that,

$$
a\left(z^{\prime}, x, \xi\right)-a(z, x, \xi)=\left(z^{\prime}-z\right)^{\alpha} \tilde{a}\left(z^{\prime}, z, x, \xi\right), 0 \leq z \leq z^{\prime} \leq Z
$$

with $\tilde{a}\left(z^{\prime}, z, x, \xi\right)$ bounded w.r.t. $z^{\prime}$ and $z$ with values in $\mathcal{M}_{k} S^{1}\left(\mathbf{R}^{n} \times \mathbf{R}^{n}\right)$.

It should be noted that Assumption 3.5 concerns the full symbol $a(z,$.$) and not simply its principal symbol.$

Proposition 3.6. Let $s \in \mathbf{R}$. There exist $\Delta_{9}>0$ and $C \geq 0$ such that for $z^{\prime}-z=\Delta, \Delta \in\left[0, \Delta_{9}\right]$,

$$
\left\|\left(\partial_{z^{\prime}}+a\left(z^{\prime}, x, D_{x}\right)\right) \circ \mathcal{G}_{\left(z^{\prime}, z\right)}\right\|_{\left(\left(H^{(s)}\right)^{k},\left(H^{(s-1)}\right)^{k}\right)} \leq C \Delta^{\alpha} .
$$

The proof is along the lines of that of Theorem 2.8 in [30] and uses the calculus result of Theorem 3.4 since in the present case phase functions are matrices. The proof can be found in Appendix A

Adapting the proof of energy estimate (2.3) to the case of piecewise $\mathscr{C}^{1}$ function yet globally Lipschitz functions like $\mathcal{W}_{\mathfrak{F}, z}\left(u_{0}\right)$ (see Proposition 3.3) we find that

$$
\left\|U(z, 0)\left(u_{0}\right)-\mathcal{W}_{\Re, z}\left(u_{0}\right)\right\|_{\left(H^{(s)}\right)^{k}} \leq C \int_{0}^{Z}\left\|\left(\partial_{z}+a\left(z, x, D_{x}\right)\right) W_{\Re, z}\left(u_{0}\right)\right\|_{\left(H^{(s)}\right)^{k}} d z,
$$

with the constant $C$ uniform w.r.t. $z$ and the subdivision $\mathfrak{P}=\left\{z^{(0)}, \ldots, z^{(N)}\right\}$, for $u_{0} \in\left(H^{(s+1)}\right)^{k}$. We take $z \in] z^{(j)}, z^{(j+1)}[$. Then

$$
\left(\partial_{z}+a\left(z, x, D_{x}\right)\right) \mathcal{W}_{\Re, z}\left(u_{0}\right)=\left(\partial_{z}+a\left(z, x, D_{x}\right)\right)\left(\mathcal{G}_{\left(z, z^{(j)}\right)} \prod_{i=j}^{1} \mathcal{G}_{\left(z^{(i)}, z^{(i-1)}\right)}\left(u_{0}\right)\right)=\left(\partial_{z}+a\left(z, x, D_{x}\right)\right)\left(\mathcal{G}_{\left(z, z^{(j)}\right)}\left(u_{j}\right)\right),
$$

with $u_{j}:=\prod_{i=j}^{1} \mathcal{G}_{\left(z^{(i)}, z^{(i-1)}\right)}\left(u_{0}\right)$ which is in $\left(H^{(s+1)}\left(\mathbf{R}^{n}\right)\right)^{k}$ by Theorem 2.22. By Proposition 3.2, the norm of $u_{j}$ in $\left(H^{(s+1)}\left(\mathbf{R}^{n}\right)\right)^{k}$ remains bounded even if $|\mathfrak{P}|=N$ becomes very large:

$$
\exists K \geq 0,\left\|u_{j}\right\|_{\left(H^{(s+1)}\right)^{k}} \leq K\left\|u_{0}\right\|_{\left(H^{(s+1)}\right)^{k}}, j \in\{0, \ldots, N\}, N=|\mathfrak{P}| \in \mathbf{N}, u_{0} \in\left(H^{(s+1)}\left(\mathbf{R}^{n}\right)\right)^{k},
$$

if $\Delta_{\mathfrak{P}}$ is small enough. By Proposition 3.6, we thus obtain

$$
\left\|\left(\partial_{z}+a\left(z, x, D_{x}\right)\right) \mathcal{W}_{\mathfrak{P}, z}\left(u_{0}\right)\right\|_{\left(H^{(s)}\right)^{k}} \leq C K \Delta_{\mathfrak{\beta}}^{\alpha}\left\|u_{0}\right\|_{\left(H^{(s+1)}\right)^{k}}, \quad z \in[0, Z] \backslash \mathfrak{P},
$$

with the constants $C$ and $K$ uniform w.r.t. $z$ and $\mathfrak{P}$.

An interpolation argument yields the main result of this Section.

Theorem 3.7. Assume that the symbol a(z,.) satisfies Assumptions 2.1 and 2.2, and is in $\mathscr{C}^{0, \alpha}\left([0, Z], \mathcal{M}_{k} S^{1}\left(\mathbf{R}^{n} \times\right.\right.$ $\left.\mathbf{R}^{n}\right)$ ), i.e. Hölder continuous w.r.t. $z$, with values in $\mathcal{M}_{k} S^{1}\left(\mathbf{R}^{n} \times \mathbf{R}^{n}\right)$, in the sense that, for some $0<\alpha<1$

$$
a\left(z^{\prime}, x, \xi\right)-a(z, x, \xi)=\left(z^{\prime}-z\right)^{\alpha} \tilde{a}\left(z^{\prime}, z, x, \xi\right), 0 \leq z \leq z^{\prime} \leq Z,
$$

or Lipschitz $(\alpha=1)$, with $\tilde{a}\left(z^{\prime}, z, x, \xi\right)$ bounded w.r.t. $z^{\prime}$ and $z$ with values in $\mathcal{M}_{k} S^{1}\left(\mathbf{R}^{n} \times \mathbf{R}^{n}\right)$. Let $s \in \mathbf{R}$ and $0 \leq r<1$. Then the approximation Ansatz $\mathcal{W}_{\mathfrak{P}, z}$ converges to the solution operator $U(z, 0)$ of the Cauchy 
problem (2.1)-(2.2) in $L\left(\left(H^{(s+1)}\left(\mathbf{R}^{n}\right)\right)^{k},\left(H^{(s+r)}\left(\mathbf{R}^{n}\right)\right)^{k}\right)$ uniformly w.r.t. $z$ as $\Delta_{\mathfrak{P}}$ goes to 0 with a convergence rate of $\operatorname{order} \alpha(1-r)$ :

$$
\left\|W_{\mathfrak{P}, z}-U(z, 0)\right\|_{\left(\left(H^{(s+1)}\right)^{k},\left(H^{(s+r)}\right)^{k}\right)} \leq C \Delta_{\mathfrak{P}}^{\alpha(1-r)}, \quad z \in[0, Z] .
$$

Furthermore, the operator $\mathcal{W}_{\mathfrak{P}, z}$ strongly converges to the solution operator $U(z, 0)$, uniformly w.r.t. $z \in$ $[0, Z]$, in $L\left(\left(H^{(s+1)}\left(\mathbf{R}^{n}\right)\right)^{k},\left(H^{(s+1)}\left(\mathbf{R}^{n}\right)\right)^{k}\right)$.

Proof. The case $r=0$ is an immediate consequence of (3.5) and (3.6).

From energy estimate (2.3) for $s+1$ we have

$$
\left\|U(z, 0)\left(u_{0}\right)\right\|_{\left(H^{(s+1)}\right)^{k}} \leq C\left\|u_{0}\right\|_{\left(H^{(s+1)}\right)^{k}} .
$$

From Proposition 3.2 we obtain

$$
\left\|\mathcal{W}_{\mathfrak{P}, z}\left(u_{0}\right)\right\|_{\left(H^{(s+1)}\right)^{k}} \leq C\left\|u_{0}\right\|_{\left(H^{(s+1)}\right)^{k}}
$$

and thus

$$
\left\|\mathcal{W}_{\mathfrak{P}, z}\left(u_{0}\right)-U(z, 0)\left(u_{0}\right)\right\|_{\left(H^{(s+1)}\right)^{k}} \leq C\left\|u_{0}\right\|_{\left(H^{(s+1)}\right)^{k}},
$$

uniformly w.r.t. $z \in[0, Z]$. The interpolation inequality

$$
\|v\|_{\left(H^{(s+r)}\right)^{k}} \leq C\|v\|_{\left(H^{(s)}\right)^{k}}^{1-r}\|v\|_{\left(H^{(s+1)}\right)^{k}}^{r}, \quad 0 \leq r \leq 1,
$$

then yields

$$
\left\|\mathcal{W}_{\mathfrak{P}, z}\left(u_{0}\right)-U(z, 0)\left(u_{0}\right)\right\|_{\left(H^{(s+r)}\right)^{k}} \leq C \Delta_{\mathfrak{P}}^{\alpha(1-r)}\left\|u_{0}\right\|_{\left(H^{(s+1)}\right)^{k}}, \quad 0 \leq r<1,
$$

uniformly w.r.t. $z \in[0, Z]$.

Let $u_{0} \in\left(H^{(s+1)}\right)^{k}$ and let $\varepsilon>0$. For the strong convergence in $\left(H^{(s+1)}\right)^{k}$ we choose $u_{1} \in\left(H^{(s+2)}\right)^{k}$ such that $\left\|u_{0}-u_{1}\right\|_{\left(H^{(s+1)}\right)^{k}} \leq \varepsilon$. We then write

$$
\begin{aligned}
\left\|\mathcal{W}_{\mathfrak{P}, z}\left(u_{0}\right)-U(z, 0)\left(u_{0}\right)\right\|_{\left(H^{(s+1)}\right)^{k}} \leq\left\|\mathcal{W}_{\mathfrak{P}, z}\left(u_{0}-u_{1}\right)\right\|_{\left(H^{(s+1)}\right)^{k}} & +\left\|\mathcal{W}_{\mathfrak{P}, z}\left(u_{1}\right)-U(z, 0)\left(u_{1}\right)\right\|_{\left(H^{(s+1)}\right)^{k}} \\
& +\left\|U(z, 0)\left(u_{0}-u_{1}\right)\right\|_{\left(H^{(s+1)}\right)^{k}} \leq C \varepsilon+C \Delta_{\mathfrak{P}}^{\alpha}\left\|u_{1}\right\|_{\left(H^{(s+2)}\right)^{k}}
\end{aligned}
$$

from estimates (3.7) and (3.8) and the case $r=0$ of the first part of the Theorem. This last estimate is uniform w.r.t. $z \in[0, Z]$ and yields the result.

\section{Symmetrizable systems}

In this section, we consider the more general situation where the matrix symbol $a_{1}$ is symmetrizable. Namely we make the following assumption.

Assumption 4.1. There exists a $k \times k$ invertible matrix $L(z, x, \xi)$ that is bounded w.r.t. $z$ with values in $\mathcal{M}_{k} S^{0}\left(X \times \mathbf{R}^{n}\right)$, homogeneous of degree zero in $\xi,|\xi| \geq 1$, with $(L(z, x, \xi))^{-1}$ satisfying the same properties, and such that

$$
a_{1}(z, x, \xi)=L(z, x, \xi) \alpha_{1}(z, x, \xi)(L(z, x, \xi))^{-1},
$$

with $\alpha_{1}=-i \beta_{1}+\gamma_{1}$ satisfying Assumptions 2.1 and 2.2.

Note that this formulation is in fact equivalent to that in which we choose $L(z, x, \xi)$ to be itself hermitian symmetric or to the formulation given in [4]: we have

$$
S(z, x, \xi) a_{1}(z, x, \xi)=\left((L(z, x, \xi))^{-1}\right)^{*} \alpha_{1}(z, x, \xi)(L(z, x, \xi))^{-1},
$$

with $S(z, x, \xi)=\left((L(z, x, \xi))^{-1}\right)^{*}(L(z, x, \xi))^{-1}$ which is hermitian symmetric and $S(z, x, \xi) \geq C \operatorname{Id}_{k}($ the converse makes use of the square root of $S$ ).

We shall make the additional following assumption. 
Assumption 4.2. The matrix symbol $L(z, x, \xi)$ is Lipschitz continuous, in the sense that

$$
L\left(z^{\prime}, x, \xi\right)-L(z, x, \xi)=\left(z^{\prime}-z\right) \tilde{L}\left(z^{\prime}, z, x, \xi\right),
$$

with $\tilde{L}\left(z^{\prime}, z, x, \xi\right)$ bounded w.r.t. $z^{\prime}$ and $z$ with values in $\mathcal{M}_{k} S^{0}\left(X \times \mathbf{R}^{n}\right)$.

The same property naturally follows for the matrix symbol $R(z, x, \xi):=(L(z, x, \xi))^{-1}$.

Example 4.3. The first-order system that describes linear anisotropic elastodynamic, written in terms of velocity and stress field, is smoothly symmetrizable if multiplicities remain constant. Similarly, Maxwell's equations are a possible application of the results of this article if multiplicities remain constants. Conical refraction in crystal optics is thus not considered here.

With the two assumptions made, the energy estimate (2.3) remains ${ }^{3}$ valid $[4$, Chapter VI] and there exists a unique solution to the Cauchy problem $(2.1)-(2.2)$ in $\mathscr{C}\left([0, Z],\left(H^{(s+1)}\left(\mathbf{R}^{n}\right)\right)^{k}\right) \cap \mathscr{C}^{1}\left([0, Z],\left(H^{(s)}\left(\mathbf{R}^{n}\right)\right)^{k}\right)$.

The thin-slab propagator $\mathcal{G}_{\left(z^{\prime}, z\right)}$ is defined as in Section 2 . We check that it satisfies the regularity properties of Proposition 2.6. The approximation Ansatz $\mathcal{W}_{\mathfrak{P}, z}$ can be defined as in Section 3. As in the previous sections, we may assume that $e^{-\Delta d_{\gamma}(z, .)}$ and $e^{-\Delta \gamma_{1}(z,)}$ take values in $\mathcal{M}_{k} S_{\rho}^{0}\left(X \times \mathbf{R}^{n}\right), \rho \in\left[\frac{1}{2}, 1\right]$ (see Lemma 2.11 and corollary 2.16).

In order to prove the stability of the Ansatz $\mathcal{W}_{\mathfrak{P}, z}$ in the case of a symmetrizable system we first need to study some basic regularity properties of scalar operators which are closely related to the composition of scalar operators of the form of

$$
\mathcal{A}_{\left(z^{\prime}, z\right)}(u)\left(x^{\prime}\right)=\iint e^{i\left\langle x^{\prime}-x \mid \xi\right\rangle} e^{-\Delta h_{1}\left(z, x^{\prime}, \xi\right)} \sigma_{\left(z^{\prime}, z\right)}\left(x^{\prime}, \xi\right) u(x) d x d \xi
$$

where $h_{1}(z, x, \xi)$ is a scalar symbol of order one.

\subsection{Some operator properties}

Let $h_{1}(z, x, \xi)$ and $\underline{h}_{1}(z, x, \xi)$ be scalar symbols that satisfy Assumption 1.1, with

$$
h_{1}(z, x, \xi)=-i k_{1}(z, x, \xi)+l_{1}(z, x, \xi), \quad \underline{h}_{1}(z, x, \xi)=-i \underline{k}_{1}(z, x, \xi)+\underline{l}_{1}(z, x, \xi),
$$

following the notation of Section 1. Let also $\sigma_{\left(z^{\prime \prime}, z^{\prime}, z\right)}\left(x^{\prime}, \xi, x, \eta\right)$ be a scalar multiple symbol bounded w.r.t. $z^{\prime \prime}, z^{\prime}$ and $z$ with values in $S_{\rho^{\prime}}^{m, m^{\prime}}\left(X^{\prime} \times \mathbf{R}^{n} \times X \times \mathbf{R}^{n}\right), \frac{1}{2} \leq \rho^{\prime} \leq 1$. As in Section 1 , we assume that $l_{1}$ and $\underline{l}_{1}$ are such that $e^{-\Delta l_{1}(z, .)}$ and $e^{-\Delta l_{1}(z,)}$ take value in $S_{\rho}^{0}\left(X \times \mathbf{R}^{n}\right), \frac{1}{2} \leq \rho \leq 1$ (see Remark 1.9 and Lemma 2.11). We assume that we have

$$
C^{\prime}\langle\eta\rangle \leq\langle\xi\rangle \leq C\langle\eta\rangle
$$

in the support of $\sigma_{\left(z^{\prime \prime}, z^{\prime}, z\right)}\left(x^{\prime}, \xi, x, \eta\right)$. Setting $\Delta=z^{\prime}-z$ and $\Delta^{\prime}=z^{\prime \prime}-z$, we formally define the operator

$$
\mathcal{F}_{\left(z^{\prime \prime}, z^{\prime}, z\right)}(u)\left(x^{\prime}\right)=\iint e^{i\left\langle x^{\prime}-x \mid \xi\right\rangle} e^{-\Delta^{\prime} h_{1}\left(z^{\prime}, x^{\prime}, \xi\right)} \iint e^{i\langle x-y \mid \eta\rangle} e^{-\Delta \underline{h}_{1}(z, x, \eta)} \sigma_{\left(z^{\prime \prime}, z^{\prime}, z\right)}\left(x^{\prime}, \xi, x, \eta\right) u(y) d y d \eta d x d \xi,
$$

with the integrations w.r.t. to the different variables performed in the order $y, \eta, x, \xi$.

Proposition 4.4. There exists $\Delta_{10}>0$ such that if $z^{\prime \prime}, z^{\prime}, z \in[0, Z]$ and $\Delta, \Delta^{\prime} \in\left[0, \Delta_{10}\right]$, then the operator $\mathcal{F}_{\left(z^{\prime \prime}, z^{\prime}, z\right)}$ is well defined on $\mathscr{P}\left(\mathbf{R}^{n}\right)$ and the map $\left(\sigma_{\left(z^{\prime \prime}, z^{\prime}, z\right)}, u\right) \mapsto \mathcal{F}_{\left(z^{\prime \prime}, z^{\prime}, z\right)}(u)$ is continuous from $S_{\rho^{\prime}}^{m, m^{\prime}}\left(X^{\prime} \times \mathbf{R}^{n} \times\right.$ $\left.X \times \mathbf{R}^{n}\right) \times \mathscr{P}\left(\mathbf{R}^{n}\right)$ into $\mathscr{P}\left(\mathbf{R}^{n}\right)$.

The proof of Proposition 4.4 can be found in Appendix A.

We now study the Sobolev-space regularity of the operator $\mathcal{F}_{\left(z^{\prime \prime}, z^{\prime}, z\right)}$.

\footnotetext{
${ }^{3}$ Note that the Lipschitz regularity is minimal to obtain the energy estimate.
} 
Proposition 4.5. Let $s \in \mathbf{R}$. The operator $\mathcal{F}_{\left(z^{\prime \prime}, z^{\prime}, z\right)}$ maps $H^{(s)}\left(\mathbf{R}^{n}\right)$ into $H^{\left(s-m-m^{\prime}\right)}\left(\mathbf{R}^{n}\right)$ and there exist $C \geq 0$ and $\Delta_{11}>0$ such that

$$
\left\|\mathcal{F}_{\left(z^{\prime \prime}, z^{\prime}, z\right)}\right\|_{\left(H^{(s)}, H^{\left(s-m-m^{\prime}\right)}\right.} \leq C p\left(\sigma_{\left(z^{\prime \prime}, z^{\prime}, z\right)}\right),
$$

for all $z^{\prime}, z \in[0, Z], \Delta^{\prime}, \Delta \in\left[0, \Delta_{11}\right]$, where $p$ is some appropriately chosen seminorm on $S_{\rho^{\prime}}^{m, m^{\prime}}\left(X^{\prime} \times \mathbf{R}^{n} \times\right.$ $\left.X \times \mathbf{R}^{n}\right)$

The proof of Proposition 4.5 can be found in Appendix A.

\subsection{Composition of two thin-slab propagators}

Because the matrix symbol $L(z, x, \xi)$ is not unitary we cannot proceed as in Section 2 and obtain an estimate for the Sobolev operator norm of $\mathcal{G}_{\left(z^{\prime}, z\right)}$ as in Theorem 2.22. The validity of such a sharp estimate in the symmetrizable case remains an open question.

We instead investigate the product $\mathcal{G}_{\left(z^{\prime \prime}, z^{\prime}\right)} \circ \mathcal{G}_{\left(z^{\prime}, z\right)}$ with $0 \leq z \leq z^{\prime} \leq z^{\prime \prime} \leq Z$, as it appears in the definition of the Ansatz $\mathcal{W}_{\Re, z}$ in Section 3.

We define the following matrix-phase FIOs

$$
\begin{aligned}
& \mathcal{H}_{\left(z^{\prime}, z\right)}^{l}(u)\left(x^{\prime}\right):=\iint e^{i\left\langle x^{\prime}-x \mid \xi\right\rangle} g_{\left(z^{\prime}, z\right)}\left(x^{\prime}, \xi\right) L\left(z, x^{\prime}, \xi\right) e^{-\Delta \alpha_{1}\left(z, x^{\prime}, \xi\right)} u(x) d x d \xi, \\
& \mathcal{H}_{\left(z^{\prime}, z\right)}^{r}(u)\left(x^{\prime}\right):=\iint e^{i\left\langle x^{\prime}-x \mid \xi\right\rangle} g_{\left(z^{\prime}, z\right)}\left(x^{\prime}, \xi\right) e^{-\Delta \alpha_{1}\left(z, x^{\prime}, \xi\right)} R\left(z, x^{\prime}, \xi\right) u(x) d x d \xi, \\
& \mathcal{H}_{\left(z^{\prime}, z\right)}(u)\left(x^{\prime}\right):=\iint e^{i\left\langle x^{\prime}-x \mid \xi\right\rangle} g_{\left(z^{\prime}, z\right)}\left(x^{\prime}, \xi\right) e^{-\Delta \alpha_{1}\left(z, x^{\prime}, \xi\right)} u(x) d x d \xi,
\end{aligned}
$$

and

$$
\mathcal{H}_{\left(z^{\prime}, z\right)}^{l r}(u)\left(x^{\prime}\right):=\mathcal{G}_{\left(z^{\prime}, z\right)}(u)\left(x^{\prime}\right), \quad u \in\left(H^{(-\infty)}\left(\mathbf{R}^{n}\right)\right)^{k} .
$$

The proofs of the following three propositions can be found in Appendix A.

Proposition 4.6. Let $s \in \mathbf{R}$. There exists an operator $\mathcal{K}_{\left(z^{\prime \prime}, z^{\prime}, z\right)}$ bounded from $\left(H^{(s)}\left(\mathbf{R}^{n}\right)\right)^{k}$ into $\left(H^{(s)}\left(\mathbf{R}^{n}\right)\right)^{k}$, uniformly w.r.t. $z^{\prime \prime}, z^{\prime}$ and $z$, such that

$$
\mathcal{H}_{\left(z^{\prime \prime}, z^{\prime}\right)}^{l r} \circ \mathcal{H}_{\left(z^{\prime}, z\right)}^{l r}=\mathcal{H}_{\left(z^{\prime \prime}, z^{\prime}\right)}^{l} \circ \mathcal{H}_{\left(z^{\prime}, z\right)}^{r}+\max \left(\Delta, \Delta^{\prime}\right) \mathcal{K}_{\left(z^{\prime \prime}, z^{\prime}, z\right)}+\mathcal{M}\left(z^{\prime \prime}, x, D_{x}\right),
$$

for $\Delta=z^{\prime}-z$ and $\Delta^{\prime}=z^{\prime \prime}-z^{\prime}$ both sufficiently small, and where

$$
\mathcal{M}\left(z, x, D_{x}\right):=\mathrm{Id}-L\left(z, x, D_{x}\right) \circ R\left(z, x, D_{x}\right) .
$$

In the sequel, we shall often write $\mathcal{M}_{z}$ in place of $\mathcal{M}\left(z, x, D_{x}\right)$ for concision.

Proposition 4.7. Let $s \in \mathbf{R}$. There exists an operator $\mathcal{K}_{\left(z^{\prime \prime}, z^{\prime}, z\right)}$ bounded from $\left(H^{(s)}\left(\mathbf{R}^{n}\right)\right)^{k}$ into $\left(H^{(s)}\left(\mathbf{R}^{n}\right)\right)^{k}$ uniformly w.r.t. $z^{\prime \prime}, z^{\prime}$ and $z$ such that

$$
\mathcal{H}_{\left(z^{\prime \prime}, z^{\prime}\right)}^{l r} \circ \mathcal{H}_{\left(z^{\prime}, z\right)}^{l}=\mathcal{H}_{\left(z^{\prime \prime}, z^{\prime}\right)}^{l} \circ \mathcal{H}_{\left(z^{\prime}, z\right)}+\max \left(\Delta^{\prime}, \Delta\right) \mathcal{K}_{\left(z^{\prime \prime}, z^{\prime}, z\right)},
$$

for $\Delta^{\prime}$ and $\Delta$ sufficiently small.

Finally, we shall use the following result.

Proposition 4.8. Let $s \in \mathbf{R}$. There exists an operator $\mathcal{K}_{\left(z^{\prime}, z\right)}$ bounded from $\left(H^{(s)}\left(\mathbf{R}^{n}\right)\right)^{k}$ into $\left(H^{(s)}\left(\mathbf{R}^{n}\right)\right)^{k}$ uniformly w.r.t. $z^{\prime}$ and $z$ such that

$$
\mathcal{H}_{\left(z^{\prime}, z\right)}^{l r} \circ \mathcal{M}\left(z, x, D_{x}\right)=\mathcal{M}\left(z^{\prime}, x, D_{x}\right)+\Delta \mathcal{K}_{\left(z^{\prime}, z\right)},
$$

for $\Delta$ sufficiently small. 


\subsection{Stability of the Ansatz $\mathcal{W}_{\mathfrak{\$}, z}$ and conclusion}

Here, to simplify the notation we shall use constant-step subdivisions $\mathfrak{P}=\left\{z^{(0)}, z^{(1)}, \ldots, z^{(N)}\right\}$ of $[0, Z]$, i.e., $\Delta_{\mathfrak{P}}=z^{(i+1)}-z^{(i)}$, for $i=0, \ldots, N-1$. The result can however be extended to more general subdivisions of $[0, Z]$.

Let $s \in \mathbf{R}$. Let $K \geq 0$. We shall denote by $\mathcal{K}$ a generic operator continuous from $\left(H^{(s)}\left(\mathbf{R}^{n}\right)\right)^{k}$ into $\left(H^{(s)}\left(\mathbf{R}^{n}\right)\right)^{k}$ such that $\|\mathcal{K}\|_{\left(\left(H^{(s)}\right)^{k},\left(H^{(s)}\right)^{k}\right)} \leq K$. We now define notations for some operators. In the notation $\mathcal{J}_{\left(z^{\left(l^{\prime}\right)}, z^{(l)}\right)}$ below, we are solely interested in the form of the operator and by its norm estimate rather than by its precise definition. Thus, in the definition of $\mathcal{J}_{\left(z^{\left(l^{\prime}\right)}, z^{(l)}\right)}$, the operators denoted by $\mathcal{K}$ may change from one term to another. We choose to make this abuse of notation for the sake of concision.

Definition 4.9. For $0 \leq l \leq l^{\prime} \leq N$, we set

$$
\begin{aligned}
& \mathcal{G}_{\left(z^{\left(l^{\prime}\right)}, \ldots, z^{\left(l^{\prime}\right)}\right)}:= \begin{cases}\operatorname{Id} & \text { if } l^{\prime}=l, \\
\mathcal{G}_{\left(z^{\left(l^{\prime}\right.}, z^{\left(l^{\prime}-1\right)}\right)} & \text { if } l^{\prime}-1=l, \\
\mathcal{G}_{\left(z^{\left(l^{\prime}\right)}, z^{\left(l^{\prime}-1\right)}\right)} \circ \cdots \circ \mathcal{G}_{\left(z^{(l+1)}, z^{(l)}\right)} & \text { otherwise, }\end{cases}
\end{aligned}
$$

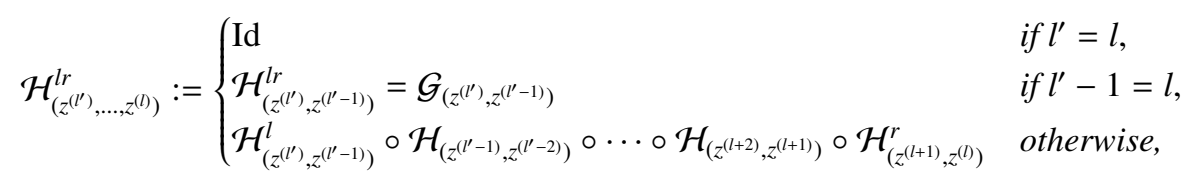

and

$$
\mathcal{H}_{\left(z^{\left(l^{\prime}\right)}, \ldots, z^{(l)}\right)}^{r}:= \begin{cases}\operatorname{Id} & \text { if } l^{\prime}=l, \\ \mathcal{H}_{\left(z^{\left(l^{\prime}\right)}, z^{\left(l^{\prime}-1\right)}\right)}^{r} & \text { if } l^{\prime}-1=l, \\ \mathcal{H}_{\left(z^{\left(l^{\prime}\right)}, z^{\left(l^{\prime}-1\right)}\right)} \circ \cdots \circ \mathcal{H}_{\left(z^{(l+2)}, z^{(l+1)}\right)} \circ \mathcal{H}_{\left(z^{(l+1)}, z^{(l)}\right)}^{r} & \text { otherwise. }\end{cases}
$$

The reader should note that $\mathcal{H}_{\left(z^{\left(l^{\prime}\right)}, z^{(l)}\right)}^{l r}=\mathcal{G}_{\left(z^{\left(l^{\prime}\right)}, z^{(l)}\right)}$ but $\mathcal{H}_{\left(z^{\left(l^{\prime}\right)}, \ldots, z^{(l)}\right)}^{l r} \neq \mathcal{G}_{\left(z^{\left(l^{\prime}\right)}, \ldots, z^{(l)}\right)}$, if $l^{\prime}-l \geq 2$. For $0 \leq l \leq l^{\prime} \leq N$, we denote by $\mathcal{J}_{\left(z^{\left(l^{\prime}\right)}, z^{(l)}\right)}$ an operator of the form $\left(\mathcal{J}_{\left(z^{(l)}, z^{(l)}\right)}=\mathrm{Id}\right)$

$$
\begin{aligned}
& \mathcal{J}_{\left(z^{\left(l^{\prime}\right)}, z^{(l)}\right)}=\mathcal{H}_{\left(z^{\left(l^{\prime}\right)}, \ldots, z^{(l)}\right)}^{l r} \\
& +\Delta_{\mathfrak{P}} \sum_{l+1 \leq m_{1} \leq l^{\prime}-1} \mathcal{H}_{\left(z^{\left(l^{\prime}\right)}, \ldots, z^{\left(m_{1}+1\right)}\right)}^{l r} \circ \mathcal{K} \circ \mathcal{H}_{\left(z^{\left(m_{1}-1\right)}, \ldots, z^{(l)}\right)}^{r} \\
& +\Delta_{\mathfrak{P}}^{r} \sum_{\substack{l+2 r-1 \leq m_{r} \leq l^{\prime}-1 \\
l+3 \leq m_{2} \leq m_{3}-2 \\
l+1 \leq m_{1} \leq m_{2}-2}} \mathcal{H}_{\left(z^{\left(l^{\prime}\right)}, \ldots, z^{\left(m_{r}+1\right)}\right)}^{l r} \circ \mathcal{K} \circ \mathcal{H}_{\left(z^{\left(m_{r}-1\right)}, \ldots, z^{\left(m_{r-1}+1\right)}\right)}^{r} \circ \cdots \mathcal{H}_{\left(z^{\left(m_{2}-1\right)}, \ldots, z^{\left(m_{1}+1\right)}\right)} \circ \mathcal{K}_{\left(z^{\left(m_{1}-1\right)}, \cdots, z^{(l)}\right)}^{r}
\end{aligned}
$$

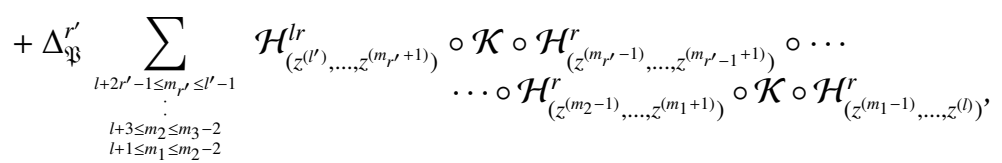

with $r^{\prime}=\mathrm{E}\left(\left(l^{\prime}-l\right) / 2\right)$.

In the sequel, the "order" of a term will refer to the power of $\Delta_{\mathfrak{P}}$ appearing in it.

Remark 4.10. Observe that in the definition of $\mathcal{J}_{\left(z^{\left(l^{\prime}\right)} z^{(l)}\right)}$, in the case where $l^{\prime}-l$ is even, the last term is in fact $\Delta_{\mathfrak{P}}^{\left(l^{\prime}-l\right) / 2} \mathcal{K} \circ \cdots \circ \mathcal{K}$, with the generic operator $\mathcal{K}$ appearing $\left(l^{\prime}-l\right) / 2$ times (we do not write $\mathcal{K}^{\left(l^{\prime}-l\right) / 2}$ since the operator $\mathcal{K}$ may not be the same each time). In the case where $l^{\prime}-l$ is odd, then, there remains one operator of the type $\mathcal{H}_{\left(z^{(m-1)}, \ldots, z^{\left(m^{\prime}+1\right)}\right)}^{r}$ or $\mathcal{H}_{\left(z^{\left(l^{\prime}\right)}, \ldots, z^{(m+1)}\right)}^{l r}$ in each term of order $\mathrm{E}\left(\left(l^{\prime}-l\right) / 2\right)=\left(l^{\prime}-1-l\right) / 2$. Basically, 
in each term in the sums above, the operator $\mathcal{K}$ replaces the occurrence of two consecutive operators of the type given in (4.3)-(4.6) and we cover all possible cases in the sums. We write the first examples of the operators $\mathcal{J}_{\left(z^{\left(l^{\prime}\right)}, z^{(l)}\right)}$ for the reader to get used to their form:

$$
\begin{aligned}
\mathcal{J}_{\left(z^{(l+1)}, z^{(l)}\right)} & =\mathcal{H}_{\left(z^{(l+1)}, z^{(l)}\right)}^{l r} \\
\mathcal{J}_{\left(z^{(l+2)}, z^{(l)}\right)} & =\mathcal{H}_{\left(z^{(l+2)}, \ldots, z^{(l)}\right)}^{l r}+\Delta_{\mathfrak{P}} \mathcal{K}, \\
\mathcal{J}_{\left(z^{(l+3)}, z^{(l)}\right)} & =\mathcal{H}_{\left(z^{(l+3)}, \ldots, z^{(l)}\right)}^{l r}+\Delta_{\mathfrak{P}}\left(\mathcal{K} \circ \mathcal{H}_{\left(z^{(l+1)}, z^{(l)}\right)}^{r}+\mathcal{H}_{\left(z^{(l+3)}, z^{(l+2)}\right)}^{l r} \circ \mathcal{K}\right), \\
\mathcal{J}_{\left(z^{(l+4)}, z^{(l)}\right)} & =\mathcal{H}_{\left(z^{(l+4)}, \ldots, z^{(l)}\right)}^{l r} \\
& +\Delta_{\mathfrak{P}}\left(\mathcal{K} \circ \mathcal{H}_{\left(z^{(l+2)}, \ldots, z^{(l)}\right)}^{r}+\mathcal{H}_{\left(z^{(l+4)}, z^{(l+3)}\right)}^{l r} \circ \mathcal{K} \circ \mathcal{H}_{\left(z^{(l+1)}, z^{(l)}\right)}^{r}+\mathcal{H}_{\left(z^{(l+4)}, \ldots, z^{(l+2)}\right)}^{l r} \circ \mathcal{K}\right) \\
& +\Delta_{\mathfrak{P}}^{2} \mathcal{K} \circ \mathcal{K},
\end{aligned}
$$

etc.

We give an estimation of the operator norm of $\mathcal{J}_{\left(z^{\left(l^{\prime}\right)}, z^{(l)}\right)}$ that relies on the sharp estimation of the Sobolev operator norm of the thin-slab propagator obtained in Theorem 2.22 in the case of a symmetric system. The result of Theorem 2.22 in fact applies to the operator $\mathcal{H}_{\left(z^{\prime}, z\right)}$, defined in (4.5), by Assumption 4.1.

The proofs of the following two lemmata can be found in Appendix A.

Lemma 4.11. There exist $S \geq 0$ and $C \geq 0$ such that

$$
\left\|\mathcal{J}_{\left(z^{\left(l^{\prime}\right)} z^{(l)}\right)}\right\|_{\left(\left(H^{(s)}\right)^{k},\left(H^{(s)}\right)^{k}\right)} \leq S^{2} e^{C Z}, \quad 0 \leq l \leq l^{\prime} \leq N,
$$

for $\Delta_{\mathfrak{P}}$ sufficiently small.

We also compute $\mathcal{H}_{\left(z^{\left(l^{\prime}+1\right)}, z^{\left(l^{\prime}\right)}\right)}^{l r} \circ \mathcal{J}_{\left(z^{\left(l^{\prime}\right)}, z^{(l)}\right)}$, which will be needed below.

Lemma 4.12. For $\Delta_{\mathfrak{P}}$ sufficiently small, i.e. $N$ large, for $l^{\prime}-l \geq 3$, we have

$$
\mathcal{H}_{\left(z^{\left(l^{\prime}+1\right)}, z^{\left(l^{\prime}\right)}\right)}^{l r} \circ \mathcal{J}_{\left(z^{\left(l^{\prime}\right)}, z^{(l)}\right)}=\mathcal{J}_{\left(z^{\left(l^{\prime}+1\right)}, z^{(l)}\right)}+\Delta_{\mathfrak{P}} \mathcal{M}_{z^{\left(l^{\prime}+1\right)}} \circ \mathcal{K} \circ \tilde{\mathcal{J}}_{\left(z^{\left(l^{\prime}-3\right)}, z^{(l)}\right)},
$$

where $\tilde{\mathcal{J}}_{\left(z^{\left(l^{\prime}\right)}, z^{(l)}\right)}$ is given by $\left(\tilde{\mathcal{J}}_{\left(z^{(l)}, z^{(l)}\right)}=\mathrm{Id}\right)$

$$
\begin{aligned}
& \tilde{\mathcal{J}}_{\left(z^{\left(l^{\prime}\right)}, z^{(l)}\right)}=\mathcal{H}_{\left(z^{\left(l^{\prime}\right)}, \ldots, z^{(l)}\right)}^{r} \\
& +\Delta_{\mathfrak{P}} \sum_{l+1 \leq m_{1} \leq l^{\prime}-1} \mathcal{H}_{\left(z^{\left(l^{\prime}\right)}, \ldots, z^{\left(m_{1}+1\right)}\right)}^{r} \circ \mathcal{K} \circ \mathcal{H}_{\left(z^{\left(m_{1}-1\right)}, \ldots, z^{(l)}\right)}^{r} \\
& +\Delta_{\mathfrak{P}}^{r} \sum_{\substack{l+2 r-1 \leq m_{r} \leq l^{\prime}-1 \\
l+3 \leq m_{2} \leq m_{3}-2 \\
l+1 \leq m_{1} \leq m_{2}-2}} \mathcal{H}_{\left(z^{\left(l^{\prime}\right)}, \ldots, z^{\left(m_{r}+1\right)}\right)}^{r} \circ \mathcal{K} \circ \mathcal{H}_{\left(z^{\left(m_{r}-1\right)}, \ldots, z^{\left(m_{r-1}+1\right)}\right)}^{r} \circ \cdots \mathcal{H}_{\left(z^{\left(m_{2}-1\right)}, \cdots, z^{\left(m_{1}+1\right)}\right)}^{r} \circ \mathcal{H}_{\left(z^{\left(m_{1}-1\right)}, \cdots, z^{(l)}\right)}^{r}
\end{aligned}
$$

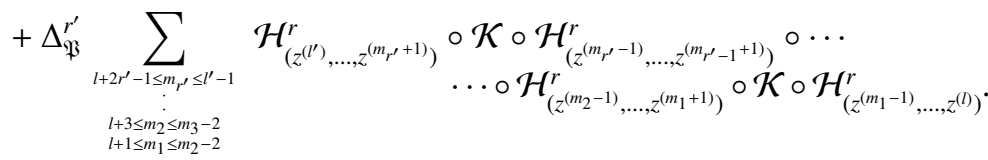

with $r^{\prime}=\mathrm{E}\left(\left(l^{\prime}-l\right) / 2\right)$. We have

$$
\left\|\tilde{\mathcal{J}}_{\left(z^{\left(l^{\prime}\right)}, z^{(l)}\right)}\right\|_{\left(\left(H^{(s)}\right)^{k},\left(H^{(s)}\right)^{k}\right)} \leq S e^{C Z}, \quad 0 \leq l \leq l^{\prime} \leq N .
$$

for the same constants $S$ and $C$ as in (4.9).

Note that the definition of $\tilde{\mathcal{J}}_{\left(z^{\left(l^{\prime}\right)}, z^{(l)}\right)}$ is similar to that of $\mathcal{J}_{\left(z^{\left(l^{\prime}\right)}, z^{(l)}\right)}$ with the terms $\mathcal{H}_{\left(z^{\prime}, \ldots, z\right)}^{l r}$ replaced by $\mathcal{H}_{\left(z^{\prime}, \ldots, z\right)}^{r}$. 
We now focus on the estimation of the operator norm of $\mathcal{G}_{\left(z^{(l)}, \ldots, z^{(0)}\right)}=\mathcal{H}_{\left(z^{(l)}, z^{(l-1)}\right)}^{l r} \circ \cdots \circ \mathcal{H}_{\left(z^{(1)}, z^{(0)}\right.}^{l r}$, i.e. the

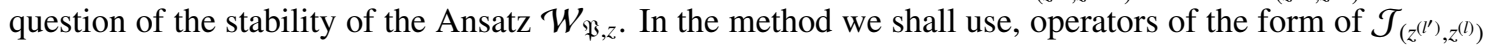
appear, for which we can now bound the operator norm uniformly w.r.t. $N=|\mathfrak{P}|$. We have seen above, in Proposition 4.6, that

$$
\begin{aligned}
\mathcal{G}_{\left(z^{(2)}, \ldots, z^{(0)}\right)} & =\mathcal{H}_{\left(z^{(2)}, z^{(1)}\right)}^{l r} \circ \mathcal{H}_{\left(z^{(1)}, z^{(0)}\right)}^{l r}=\mathcal{H}_{\left(z^{(2)}, z^{(1)}\right)}^{l} \circ \mathcal{H}_{\left(z^{(1)}, z^{(0)}\right)}^{r}+\Delta_{\mathfrak{P}} \mathcal{K}+\mathcal{M}_{z^{(2)}} \\
& =\mathcal{H}_{\left(z^{(2)}, \ldots, z^{(0)}\right)}^{l}+\Delta_{\mathfrak{P}} \mathcal{K}+\mathcal{M}_{z^{(2)}}=\mathcal{J}_{\left(z^{(2)}, z^{(0)}\right)}+\mathcal{M}_{z^{(2)}} .
\end{aligned}
$$

Composing with $\mathcal{H}_{\left(z^{(3)}, z^{(2)}\right)}^{l r}$ on the 1.h.s. we obtain

$$
\begin{aligned}
\mathcal{G}_{\left(z^{(3)}, \ldots, z^{(0)}\right)} & =\mathcal{H}_{\left(z^{(3)}, z^{(2)}\right)}^{l r} \circ \mathcal{H}_{\left(z^{(2)}, z^{(1)}\right)}^{l r} \circ \mathcal{H}_{\left(z^{(1)}, z^{(0)}\right)}^{l r}=\mathcal{H}_{\left(z^{(3)}, z^{(2)}\right)}^{l r} \circ\left(\mathcal{H}_{\left(z^{(2)}, \ldots, z^{(0)}\right)}^{l r}+\Delta_{\mathfrak{P}} \mathcal{K}+\mathcal{M}_{z^{(2)}}\right) \\
& =\mathcal{H}_{\left(z^{(3)}, \ldots, z^{(0)}\right)}^{l r}+\Delta_{\mathfrak{F}}\left(\mathcal{K} \circ \mathcal{H}_{\left(z^{(1)}, z^{(0)}\right)}^{r}+\mathcal{H}_{\left(z^{(3)}, z^{(2)}\right)}^{l r} \circ \mathcal{K}\right)+\mathcal{H}_{\left(z^{(3)}, z^{(2)}\right)}^{l r} \circ \mathcal{M}_{z^{(2)}} \\
& =\mathcal{J}_{\left(z^{(3)}, z^{(0)}\right)}+\mathcal{H}_{\left(z^{(3)}, z^{(2)}\right)}^{r} \circ \mathcal{M}_{z^{(2)}}=\mathcal{J}_{\left(z^{(3)}, z^{(0)}\right)}+\mathcal{M}_{z^{(3)}}+\Delta_{\mathfrak{P}} \mathcal{K},
\end{aligned}
$$

by Proposition 4.7 and Proposition 4.8. We carry on with these explicit computations to derive the form of $\mathcal{G}_{\left(z^{(5)}, \ldots, z^{(0)}\right)}=\mathcal{H}_{\left(z^{(5)}, z^{(4)}\right)}^{l r} \circ \cdots \circ \mathcal{H}_{\left(z^{(1)}, z^{(0)}\right)}^{l r}$. We have

$$
\begin{aligned}
\mathcal{G}_{\left(z^{(4)}, \ldots, z^{(0)}\right)} & =\mathcal{H}_{\left(z^{(4)}, z^{(3)}\right)}^{l r} \circ \cdots \circ \mathcal{H}_{\left(z^{(1)}, z^{(0)}\right)}^{l r}=\mathcal{H}_{\left(z^{(4)}, z^{(3)}\right)}^{l r} \circ\left(\mathcal{J}_{\left(z^{(3)}, z^{(0)}\right)}+\mathcal{M}_{z^{(3)}}+\Delta_{\mathfrak{P}} \mathcal{K}\right) \\
& =\mathcal{J}_{\left(z^{(4)}, z^{(0)}\right)}+\Delta_{\mathfrak{F}} \mathcal{M}_{z^{(4)}} \circ \mathcal{K} \circ \tilde{\mathcal{J}}_{\left.z^{(0)}, z^{(0)}\right)}+\mathcal{M}_{z^{(4)}}+\Delta_{\mathfrak{F}} \mathcal{K}+\Delta_{\mathfrak{F}} \mathcal{H}_{\left.z^{(4)}, z^{(3)}\right)}^{l r} \circ \mathcal{K} \\
& =\mathcal{J}_{\left(z^{(4)}, z^{(0)}\right)}+\mathcal{M}_{z^{(4)}} \circ\left(\operatorname{Id}+\Delta_{\mathfrak{F}} \mathcal{K} \circ \tilde{\mathcal{J}}_{\left(z^{(0)}, z^{(0)}\right)}\right)+\Delta_{\mathfrak{F}} \mathcal{K}+\Delta_{\mathfrak{F}} \mathcal{H}_{\left(z^{(4)}, z^{(3)}\right)}^{l r} \circ \mathcal{K},
\end{aligned}
$$

by Proposition 4.7 and Proposition 4.8 and Lemma 4.12. Similarly, we obtain

$$
\begin{aligned}
& \mathcal{G}_{\left(z^{(5)}, \ldots, z^{(0)}\right)}=\mathcal{H}_{\left(z^{(5)}, z^{(4)}\right)}^{l r} \circ \cdots \circ \mathcal{H}_{\left(z^{(1)}, z^{(0)}\right)}^{l r} \\
& =\mathcal{H}_{\left(z^{(5)}, z^{(4)}\right)}^{l r} \circ\left[\mathcal{J}_{\left(z^{(4)}, z^{(0)}\right)}+\mathcal{M}_{z^{(4)}} \circ\left(\operatorname{Id}+\Delta_{\mathfrak{F}} \mathcal{K} \circ \tilde{\mathcal{J}}_{\left(z^{(0)}, z^{(0)}\right)}\right)+\Delta_{\mathfrak{F}} \mathcal{K}+\Delta_{\mathfrak{F}} \mathcal{H}_{\left(z^{(4)}, z^{(3)}\right)}^{l r} \circ \mathcal{K}\right] \\
& =\mathcal{J}_{\left(z^{(5)}, z^{(0)}\right)}+\Delta_{\mathfrak{P}} \mathcal{M}_{z^{(5)}} \circ \mathcal{K} \circ \tilde{\mathcal{J}}_{\left(z^{(1)}, z^{(0)}\right)}+\left(\mathcal{M}_{z^{(5)}}+\Delta_{\mathfrak{F}} \mathcal{K}\right) \circ\left(\operatorname{Id}+\Delta_{\mathfrak{P}} \mathcal{K} \circ \tilde{\mathcal{J}}_{\left(z^{(0)}, z^{(0)}\right)}\right) \\
& +\Delta_{\mathfrak{P}}\left(\mathcal{H}_{\left(z^{(5)}, z^{(4)}\right)}^{l r} \circ \mathcal{K}+\mathcal{H}_{\left(z^{(5)}, z^{(4)}\right)}^{l r} \circ \mathcal{H}_{\left(z^{(4)}, z^{(3)}\right)}^{l r} \circ \mathcal{K}\right) \\
& =\mathcal{J}_{\left(z^{(5)}, z^{(0)}\right)}+\Delta_{\mathfrak{P}} \mathcal{G}_{\left(z^{(5)}, \ldots, z^{(3)}\right)} \circ \mathcal{K}+\mathcal{M}_{z^{(5)}} \circ\left(\operatorname{Id}+\Delta_{\mathfrak{F}} \mathcal{K} \circ \tilde{\mathcal{J}}_{\left(z^{(0)}, z^{(0)}\right)}+\Delta_{\mathfrak{F}} \mathcal{K} \circ \tilde{\mathcal{J}}_{\left(z^{(1)}, z^{(0)}\right)}\right) \\
& +\Delta_{\mathfrak{F}} \mathcal{G}_{\left(z^{(5)}, z^{(4)}\right)} \circ \mathcal{K}+\Delta_{\mathfrak{P}} \mathcal{K} \circ\left(\mathrm{Id}+\Delta_{\mathfrak{P}} \mathcal{K} \circ \tilde{\mathcal{J}}_{\left(z^{(0)}, z^{(0)}\right)}\right) .
\end{aligned}
$$

By induction we can now obtain

Lemma 4.13. Let $l \geq 5$, we have

$$
\begin{aligned}
\mathcal{G}_{\left(z^{(l)}, \ldots, z^{(0)}\right)}=\mathcal{J}_{\left(z^{(l)}, z^{(0)}\right)}+\Delta_{\mathfrak{F}} \mathcal{G}_{\left(z^{(l)}, \ldots, z^{\left(\mathcal{B}^{3}\right)}\right.} \circ \mathcal{K} & +\mathcal{M}_{z^{(l)}} \circ\left(\operatorname{Id}+\Delta_{\mathfrak{F}} \sum_{j=0}^{l-4} \mathcal{K} \circ \tilde{\mathcal{J}}_{\left(z^{(j)}, z^{(0)}\right)}\right) \\
& +\Delta_{\mathfrak{P}} \sum_{j=4}^{l}\left(\mathcal{G}_{\left(z^{(l)}, \ldots, z^{(j)}\right)} \circ \mathcal{K} \circ\left(\operatorname{Id}+\Delta_{\mathfrak{P}} \sum_{i=0}^{j-5} \mathcal{K} \circ \tilde{\mathcal{J}}_{\left(z^{(i)}, z^{(0)}\right.}\right)\right),
\end{aligned}
$$

with the convention $\sum_{i=0}^{-1} \mathcal{K} \circ \tilde{\mathcal{J}}_{\left(z^{(i)}, z^{(0)}\right)}=0$.

Proof. Formula (4.11) holds for $l=5$, as we have seen above. For $l \geq 5$, we now write

$$
\begin{aligned}
\mathcal{G}_{\left(z^{(l+1)}, \ldots, z^{(0)}\right)}= & \mathcal{G}_{\left(z^{(l+1)}, z^{(l)}\right)} \circ \mathcal{G}_{\left(z^{(l)}, \ldots, z^{(0)}\right)} \\
= & \mathcal{G}_{\left(z^{(l+1)}, z^{(l)}\right)} \circ\left[\mathcal{J}_{\left(z^{(l)}, z^{(0)}\right)}+\Delta_{\mathfrak{P}} \mathcal{G}_{\left(z^{(l)}, \ldots, z^{(3)}\right)} \circ \mathcal{K}+\mathcal{M}_{z^{(l)}} \circ\left(\operatorname{Id}+\Delta_{\mathfrak{P}} \sum_{j=0}^{l-4} \mathcal{K} \circ \tilde{\mathcal{J}}_{\left(z^{(j)}, z^{(0)}\right)}\right)\right. \\
& \left.+\Delta_{\mathfrak{P}} \sum_{j=4}^{l}\left(\mathcal{G}_{\left(z^{(l)}, \ldots, z^{(j)}\right)} \circ \mathcal{K} \circ\left(\operatorname{Id}+\Delta_{\mathfrak{P}} \sum_{i=0}^{j-5} \mathcal{K} \circ \tilde{\mathcal{J}}_{\left(z^{(i)}, z^{(0)}\right)}\right)\right)\right] .
\end{aligned}
$$


We have

$$
\mathcal{G}_{\left(z^{(l+1)}, z^{(l)}\right)} \circ \mathcal{J}_{\left(z^{(l)}, z^{(0)}\right)}=\mathcal{J}_{\left(z^{(l+1)}, z^{(0)}\right)}+\Delta_{\mathfrak{P}} \mathcal{M}_{z^{(l+1)}} \circ \mathcal{K} \circ \tilde{\mathcal{J}}_{\left(z^{(l-3)}, z^{(0)}\right)},
$$

by Lemma 4.12. Next, by definition we have

$$
\mathcal{G}_{\left(z^{(l+1)}, z^{(l)}\right)} \circ\left(\Delta_{\mathfrak{P}} \mathcal{G}_{\left(z^{(l)}, \ldots, z^{(3)}\right)} \circ \mathcal{K}\right)=\Delta_{\mathfrak{P}} \mathcal{G}_{\left(z^{(l+1)}, \ldots, z^{(3)}\right)} \circ \mathcal{K} .
$$

By Proposition 4.8, we obtain

$$
\mathcal{G}_{\left(z^{(l+1)}, z^{(l)}\right)} \circ \mathcal{M}_{z^{(l)}} \circ\left(\mathrm{Id}+\Delta_{\mathfrak{P}} \sum_{j=0}^{l-4} \mathcal{K} \circ \tilde{\mathcal{J}}_{\left(z^{(j)}, z^{(0)}\right)}\right)=\left(\mathcal{M}_{z^{(l+1)}}+\Delta_{\mathfrak{P}} \mathcal{K}\right) \circ\left(\operatorname{Id}+\Delta_{\mathfrak{P}} \sum_{j=0}^{l-4} \mathcal{K} \circ \tilde{\mathcal{J}}_{\left(z^{(j)}, z^{(0)}\right)}\right)
$$

Finally we have

$$
\begin{aligned}
\mathcal{G}_{\left(z^{(l+1)}, z^{(l)}\right)} \circ \Delta_{\mathfrak{P}} \sum_{j=4}^{l}\left(\mathcal{G}_{\left(z^{(l)}, \ldots, z^{(j)}\right)} \circ \mathcal{K} \circ(\operatorname{Id}\right. & \left.\left.+\Delta_{\mathfrak{P}} \sum_{i=0}^{j-5} \mathcal{K} \circ \tilde{\mathcal{J}}_{\left(z^{(i)}, z^{(0)}\right)}\right)\right) \\
& =\Delta_{\mathfrak{P}} \sum_{j=4}^{l}\left(\mathcal{G}_{\left(z^{(l+1)}, \ldots, z^{(j)}\right)} \circ \mathcal{K} \circ\left(\operatorname{Id}+\Delta_{\mathfrak{P}} \sum_{i=0}^{j-5} \mathcal{K} \circ \tilde{\mathcal{J}}_{\left(z^{(i)}, z^{(0)}\right)}\right)\right) .
\end{aligned}
$$

Since $\mathcal{G}_{\left(z^{(l+1)}, \ldots, z^{(l+1)}\right)}=\mathrm{Id}$, we have

$$
\Delta_{\mathfrak{P}} \mathcal{K} \circ\left(\operatorname{Id}+\Delta_{\mathfrak{P}} \sum_{j=0}^{l-4} \mathcal{K} \circ \tilde{\mathcal{J}}_{\left(z^{(j)}, z^{(0)}\right)}\right)=\Delta_{\mathfrak{P}} \mathcal{G}_{\left(z^{(l+1)}, \ldots, z^{(l+1)}\right)} \circ \mathcal{K} \circ\left(\operatorname{Id}+\Delta_{\mathfrak{P}} \sum_{i=0}^{l+1-5} \mathcal{K} \circ \tilde{\mathcal{J}}_{\left(z^{(i)}, z^{(0)}\right)}\right)
$$

which yields formula (4.11) at the order $l+1$, when summing (4.12)-(4.15) together.

From Lemma 4.12 we observe that

$$
\left\|\operatorname{Id}+\Delta_{\mathfrak{P}} \sum_{j=0}^{l} \mathcal{K} \circ \tilde{\mathcal{J}}_{\left(z^{(j)}, z^{(0)}\right)}\right\|_{\left(\left(H^{(s)}\right)^{k},\left(H^{(s)}\right)^{k}\right)} \leq C, \quad 0 \leq l \leq N,
$$

with $C$ uniform w.r.t. $\mathfrak{P}$ and $l$, since $\Delta_{\mathfrak{P}}=Z / N$. As $\left\|\mathcal{M}_{z}\right\|_{\left(\left(H^{(s)}\right)^{k},\left(H^{(s)}\right)^{k}\right)}$ and $\left\|\mathcal{J}_{\left(z^{(l)}, z^{(0)}\right)}\right\|_{\left(\left(H^{(s)}\right)^{k},\left(H^{(s)}\right)^{k}\right)}$ are bounded uniformly w.r.t. $z$ and $l$, we obtain the existence of $A \geq 0$ and $B \geq 0$ such that

$$
\left\|\mathcal{G}_{\left(z^{(l)}, z^{(0)}\right)}\right\|_{\left(\left(H^{(s)}\right)^{k},\left(H^{(s)}\right)^{k}\right)} \leq A+\Delta_{\mathfrak{P}} B \sum_{j=3}^{l}\left\|\mathcal{G}_{\left(z^{(l)}, z^{(j)}\right)}\right\|_{\left(\left(H^{(s)}\right)^{k},\left(H^{(s)}\right)^{k}\right)},
$$

from Lemma 4.13, which gives, with $V_{l^{\prime}, l}=\left\|\mathcal{G}_{\left(z^{\left(l^{\prime}\right)}, z^{(l)}\right)}\right\|_{\left(\left(H^{(s)}\right)^{k},\left(H^{(s)}\right)^{k}\right)}$,

$$
V_{l, 0} \leq A+\Delta_{\mathfrak{P}} B \sum_{j=3}^{l} V_{l, j} \leq A+\Delta_{\mathfrak{P}} B \sum_{j=1}^{l} V_{l, j}
$$

Above, we have chosen to use $z^{(0)}=0$ as the starting value for $z$. However, similarly, we obtain

$$
V_{l^{\prime}, l} \leq A+\Delta_{\mathfrak{P}} B \sum_{j=l+1}^{l^{\prime}} V_{l^{\prime}, j}, \quad 0 \leq l \leq l^{\prime} \leq N .
$$

Define the finite sequence $\left(W_{l}\right)_{0 \leq l \leq N}$ by

$$
W_{0}=1, \quad W_{l+1}=A+\Delta_{\mathfrak{P}} B \sum_{j=0}^{l} W_{j}, \quad 0 \leq l \leq N-1 .
$$


Since $\mathcal{G}_{\left(z^{(l)}, z^{(l)}\right)}=\mathrm{Id}, 0 \leq l \leq N$, we have $V_{l, l}=1$ and a simple induction gives

$$
V_{l^{\prime}, l} \leq W_{l^{\prime}-l}, \quad 0 \leq l \leq l^{\prime} \leq N .
$$

We now observe that for all $2 \leq l \leq N$,

$$
\begin{aligned}
W_{l} & =W_{l-1}+\Delta_{\mathfrak{F}} B W_{l-1}=\left(1+\Delta_{\mathfrak{F}} B\right) W_{l-1}=\left(1+\Delta_{\mathfrak{F}} B\right)^{l} W_{1}=\left(1+\Delta_{\mathfrak{F}} B\right)^{l}\left(A+\Delta_{\mathfrak{F}} B\right) \\
& \leq\left(1+\frac{B Z}{N}\right)^{N}\left(A+\Delta_{\mathfrak{F}} B\right) \leq C e^{B Z} .
\end{aligned}
$$

For the Ansatz $\mathcal{W}_{\mathfrak{P}, z}$ in the symmetrizable case, we thus have the following counterpart to Proposition 3.2.

Theorem 4.14. Let $s \in \mathbf{R}$. Under Assumptions 4.1 and 4.2, there exists $K^{\prime} \geq 0$ such that for every subdivision $\mathfrak{P}=\left\{z^{(0)}, z^{(1)}, \ldots, z^{(N)}\right\}$ of $[0, Z]$ with $0=z^{(0)}<z^{(1)}<\cdots<z^{(N)}=Z, \Delta_{\mathfrak{P}}=z^{(i+1)}-z^{(i)}$, for

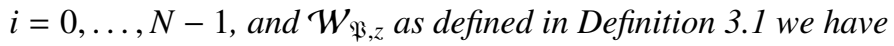

$$
\forall z \in[0, Z],\left\|\mathcal{W}_{\Re, z}\right\|_{\left(\left(H^{(s)}\right)^{k},\left(H^{(s)}\right)^{k}\right)} \leq K^{\prime},
$$

for $\Delta_{\mathfrak{F}}$ sufficiently small.

With the stability of $\mathcal{W}_{\Re, z}$ established, we can proceed with the analysis of its convergence as in Section 3. There is no difference in the argumentation between the symmetric and the symmetrizable cases there. We thus obtain a theorem similar to Theorem 3.7, which gives a representation of the solution operator of the Cauchy problem (2.1)-(2.2) by an infinite product of matrix-phase FIOs.

Theorem 4.15. Let Assumptions 4.1 and 4.2 hold and let further assume that the symbol $a(z,$.$) belongs to$ $\mathscr{C}^{0, \alpha}\left([0, Z], \mathcal{M}_{k} S^{1}\left(\mathbf{R}^{n} \times \mathbf{R}^{n}\right)\right)$, i.e. Hölder continuous w.r.t. $z$, with values in $\mathcal{M}_{k} S^{1}\left(\mathbf{R}^{n} \times \mathbf{R}^{n}\right)$, in the sense that, for some $0<\alpha<1$

$$
a\left(z^{\prime}, x, \xi\right)-a(z, x, \xi)=\left(z^{\prime}-z\right)^{\alpha} \tilde{a}\left(z^{\prime}, z, x, \xi\right), 0 \leq z \leq z^{\prime} \leq Z,
$$

or Lipschitz $(\alpha=1)$, with $\tilde{a}\left(z^{\prime}, z, x, \xi\right)$ bounded w.r.t. $z^{\prime}$ and $z$ with values in $\mathcal{M}_{k} S^{1}\left(\mathbf{R}^{n} \times \mathbf{R}^{n}\right)$. Let $s \in \mathbf{R}$ and $0 \leq r<1$. Then the approximation Ansatz $\mathcal{W}_{\mathfrak{P}, z}$ converges to the solution operator $U(z, 0)$ of the Cauchy problem (2.1)-(2.2) in $L\left(\left(H^{(s+1)}\left(\mathbf{R}^{n}\right)\right)^{k},\left(H^{(s+r)}\left(\mathbf{R}^{n}\right)\right)^{k}\right)$ uniformly w.r.t. $z$ as $\Delta_{\mathfrak{P}}$ goes to 0 with a convergence rate of order $\alpha(1-r)$ :

$$
\left\|\mathcal{W}_{\mathfrak{P}, z}-U(z, 0)\right\|_{\left(\left(H^{(s+1)}\right)^{k},\left(H^{(s+r)}\right)^{k}\right)} \leq C \Delta_{\mathfrak{P}}^{\alpha(1-r)}, \quad z \in[0, Z] .
$$

Furthermore, the operator $\mathcal{W}_{\mathfrak{P}, z}$ strongly converges to the solution operator $U(z, 0)$ uniformly w.r.t. $z \in$ $[0, Z]$ in $L\left(\left(H^{(s+1)}\left(\mathbf{R}^{n}\right)\right)^{k},\left(H^{(s+1)}\left(\mathbf{R}^{n}\right)\right)^{k}\right)$.

\section{A Some technical proofs}

\section{A.1 Proof of Proposition 1.6}

For the proof of Proposition 1.6 we shall need the following lemma.

Lemma A.1. Let $j$, $r$ be non-negative integers, $u \in \mathscr{P}\left(\mathbf{R}^{n}\right), f \in \mathscr{C}^{r+1}\left(\mathbf{R}^{n}\right)$ such that

$$
0 \leq \operatorname{Im} f(x) \leq C_{0}, x \in \mathbf{R}^{n}, \quad\left|f^{(s)}(x)\right| \leq C_{s}, x \in \mathbf{R}^{n}, 1 \leq s \leq r+1 .
$$

Then we have

$$
\omega^{j+r}\left|\int u(x)(\operatorname{Im} f(x))^{j} e^{i \omega f(x)} d x\right| \leq C \sum_{|\alpha| \leq r} \sup _{x \in \mathbf{R}^{n}}\left|D_{x}^{\alpha} u(x)\right|\left(\left|f^{\prime}(x)\right|^{2}+\operatorname{Im} f(x)\right)^{|\alpha| / 2-r}, \omega>0,
$$

where the constant $C=C(f)$ is bounded when the function $f$ stays in a domain of $\mathscr{C}^{r+1}\left(\mathbf{R}^{n}\right)$ where $C_{0}$, $C_{1}, \ldots, C_{r+1}$ can be chosen bounded. 
Proof. The proof is the same as that of Theorem 7.7.1 in [16] where $u \in \mathscr{C}_{c}^{r}\left(\mathbf{R}^{n}\right)$. In fact the further assumptions on $f$ made here allow to give global bounds that are needed since $u \in \mathscr{P}\left(\mathbf{R}^{n}\right)$ in the present case.

Proof of Proposition 1.6. At first we shall assume that $u \in \mathscr{C}_{c}^{\infty}\left(\mathbf{R}^{n}\right)$. As the operator $\mathcal{A}_{\left(z^{\prime}, z\right)}$ is a global FIO we then have $\mathcal{A}_{\left(z^{\prime}, z\right)}(u) \in \mathscr{C}^{\infty}\left(\mathbf{R}^{n}\right)$ [14, Section 25.2]. Differentiation of $\mathcal{A}_{\left(z^{\prime}, z\right)}(u)(x)$ w.r.t. to the variable $x$ is performed as usual for oscillatory integrals [16, end of Section 7.8]. Define

$$
v(\xi, \eta, y):=\int e^{-i\langle x \mid \xi\rangle-\Delta h_{1}(z, y, x, \xi)} \sigma_{\left(z^{\prime}, z\right)}(y, x, \eta) u(x) d x,
$$

and set $w(\xi, y):=v(\xi, \xi, y)$. We then have

$$
\mathcal{A}_{\left(z^{\prime}, z\right)}(u)\left(x^{\prime}\right)=\left.\tilde{w}\left(x^{\prime}, y\right)\right|_{y=x^{\prime}}, \text { with } \tilde{w}\left(x^{\prime}, y\right):=\int e^{i\left\langle x^{\prime} \mid \xi\right\rangle} w(\xi, y) d \xi .
$$

We shall first prove that $y \mapsto w(\xi, y)$ is bounded w.r.t. to $y \in \mathbf{R}^{n}$ with values in $\mathscr{P}\left(\mathbf{R}^{n}\right)$. Let $\omega=|\xi| \geq 1$ and $\xi_{0}=\xi /|\xi| \in \mathcal{S}^{n-1}$. We then have $-\langle x \mid \xi\rangle+i \Delta h_{1}(z, y, x, \xi)=\omega f(z, y, x, \xi)$ with $f$ homogeneous of degree zero in $\xi$, for $|\xi| \geq 1$. Note that $\partial_{x} f(z, y, x, \xi)=-\xi_{0}+i \Delta \partial_{x} h_{1}\left(z, y, x, \xi_{0}\right)$. Uniformly w.r.t. $z \in[0, Z]$ and w.r.t. $y, x \in \mathbf{R}^{n}$ we have $\left|\partial_{x} f(z, y, x, \xi)\right| \geq c>0$, for $\Delta$ sufficiently small. Applying Lemma A.1 and estimate (A.1) we obtain, for $r \in \mathbf{N}$,

$$
\omega^{r}|v(\xi, \eta, y)| \leq K_{r} \sum_{|\alpha| \leq r} \sup _{x \in \mathbf{R}^{n}}\left|D_{x}^{\alpha}\left(\sigma_{\left(z^{\prime}, z\right)}(y, x, \eta) u(x)\right)\right| \leq K_{r}^{\prime}\langle\eta\rangle^{m+\delta r} q_{r}\left(\sigma_{\left(z^{\prime}, z\right)}\right) \sup _{\substack{|\alpha| \leq r \\ x \in \mathbf{R}^{n}}}\left|D_{x}^{\alpha} u(x)\right|, \quad \omega \geq 1,
$$

where $q_{r}$ is a seminorm on $S_{\rho}^{m}$, and where the constants $K_{r}, K_{r}^{\prime}$ can be chosen uniformly w.r.t. $z, y$ and $\xi$, $|\xi| \geq 1$ since the constants $C_{0}, C_{1}, \ldots, C_{r+1}$ of Lemma A.1 can be chosen bounded (as $\xi_{0} \in \mathcal{S}^{n-1}$ ). Now setting $\eta=\xi$ we obtain that for all $r \in \mathbf{N}, \exists K_{r}^{\prime \prime} \geq 0$

$$
\langle\xi\rangle^{r(1-\delta)-m}|w(\xi, y)| \leq K_{r}^{\prime \prime} q_{r}\left(\sigma_{\left(z^{\prime}, z\right)}\right) \sup _{\substack{|\alpha| \leq r \\ x \in \mathbf{R}^{n}}}\left|D_{x}^{\alpha} u(x)\right|, \quad \xi \in \mathbf{R}^{n},|\xi| \geq 1, y \in \mathbf{R}^{n} .
$$

We now consider

$$
\partial_{\xi_{i}} w(\xi, y)=\int e^{-i\langle x \mid \xi\rangle-\Delta h_{1}(z, y, x, \xi)}\left(\partial_{\xi_{i}} \sigma_{\left(z^{\prime}, z\right)}(y, x, \xi)+\sigma_{\left(z^{\prime}, z\right)}(y, x, \xi)\left(-i x_{i}-\Delta \partial_{\xi_{i}} h_{1}(z, y, x, \xi)\right)\right) u(x) d x .
$$

As $x_{i} u(x) \in \mathscr{C}_{c}^{\infty}\left(\mathbf{R}^{n}\right)$ and $\partial_{\xi_{i}} h_{1}(z, y, x, \xi)$ is homogeneous of degree zero in $\xi$ for $|\xi| \geq 1$ estimates similar to those in (A.2) are valid. Thus $y \mapsto w(\xi, y)$ is bounded w.r.t. to $y \in \mathbf{R}^{n}$ with values in $\mathscr{P}\left(\mathbf{R}^{n}\right)$.

Then, $\tilde{w}\left(x^{\prime}, y\right)$ obtained by Fourier transformation is also bounded w.r.t. to $y \in \mathbf{R}^{n}$ with values in $\mathscr{P}\left(\mathbf{R}^{n}\right)$ and we have, with a multi-index $\alpha$,

$$
\sup _{x^{\prime} \in \mathbf{R}^{n}}\left|x^{\prime \alpha} \mathcal{A}_{\left(z^{\prime}, z\right)}(u)\left(x^{\prime}\right)\right| \leq \sup _{y \in \mathbf{R}^{n}} \sup _{x^{\prime} \in \mathbf{R}^{n}}\left|x^{\prime \alpha} \tilde{w}\left(x^{\prime}, y\right)\right| \leq C q_{\alpha}\left(\sigma_{\left(z^{\prime}, z\right)}\right) p_{\alpha}(u),
$$

for $p_{\alpha}$ a seminorm on $\mathscr{P}\left(\mathbf{R}^{n}\right), q_{\alpha}$ a seminorm on $S_{\rho}^{m}$, because of (A.2) and the continuity of the Fourier transformation on $\mathscr{P}\left(\mathbf{R}^{n}\right)$.

Differentiating $\mathcal{A}_{\left(z^{\prime}, z\right)}(u)\left(x^{\prime}\right)$ w.r.t. to $x_{i}^{\prime}, i=1, \ldots, n$, we obtain

$$
\partial_{x_{i}^{\prime}} \mathcal{A}_{\left(z^{\prime}, z\right)}(u)\left(x^{\prime}\right)=\int e^{i\left\langle x^{\prime}-x \mid \xi\right\rangle} e^{-\Delta h_{1}\left(z, x^{\prime}, x, \xi\right)}\left(\partial_{x_{i}^{\prime}} \sigma_{\left(z^{\prime}, z\right)}\left(x^{\prime}, x, \xi\right)+\sigma_{\left(z^{\prime}, z\right)}\left(x^{\prime}, x, \xi\right)\left(i \xi_{i}-\Delta \partial_{x_{i}^{\prime}} h_{1}\left(z, x^{\prime}, x, \xi\right)\right)\right) d \xi,
$$

and the same method yields, by induction, for $\alpha$ and $\beta$ multi-indices,

$$
\sup _{x^{\prime} \in \mathbf{R}^{n}}\left|x^{\prime \alpha} \partial_{x^{\prime}}^{\beta} \mathcal{A}_{\left(z^{\prime}, z\right)}(u)\left(x^{\prime}\right)\right| \leq C q_{\alpha, \beta}\left(\sigma_{\left(z^{\prime}, z\right)}\right) p_{\alpha, \beta}(u),
$$

for $p_{\alpha, \beta}$ a seminorm on $\mathscr{P}\left(\mathbf{R}^{n}\right)$ and $q_{\alpha, \beta}$ a seminorm on $S_{\rho}^{m}$. We can now relax the assumption made on $u$, i.e., $u \in \mathscr{C}_{c}^{\infty}\left(\mathbf{R}^{n}\right)$, by a density argument. Hence the operator $\mathcal{A}_{\left(z^{\prime}, z\right)}$ maps $\mathscr{P}\left(\mathbf{R}^{n}\right)$ into $\mathscr{P}\left(\mathbf{R}^{n}\right)$ continuously. Observe now that the adjoint of $\mathcal{A}_{\left(z^{\prime}, z\right)}$ is of the same form and thus maps $\mathscr{P}\left(\mathbf{R}^{n}\right)$ into $\mathscr{P}\left(\mathbf{R}^{n}\right)$ continuously. This concludes the proof. 


\section{A.2 Proof of Proposition 1.10}

As mentioned in the main text we only prove item 2 of the proposition here.

We set $\tilde{\rho}=\min \left(\rho, \rho^{\prime}, \rho^{\prime \prime}\right)$ and $\tilde{\delta}=\max \left(\delta, \delta^{\prime}, \delta^{\prime \prime}\right)$ and

$$
\rho_{\left(z^{\prime}, z\right)}\left(x^{\prime}, \xi, y, \eta\right):=e^{-\Delta l_{1}\left(z, x^{\prime}, y, \xi\right)} \sigma_{\left(z^{\prime}, z\right)}\left(x^{\prime}, y, \xi\right) p(y, \eta)
$$

We observe that it is a multiple symbol in $S_{\tilde{\rho}, \tilde{\delta}}^{m, m^{\prime}}\left(X^{\prime} \times \mathbf{R}^{n} \times Y \times \mathbf{R}^{n}\right)$. Expression (1.4) can be formally obtained. We set

$$
\varphi_{\left(z^{\prime}, z\right)}\left(x^{\prime}, x, y, \xi, \eta\right):=\langle x-y \mid \xi-\eta\rangle+\Delta\left(k_{1}\left(z, x^{\prime}, y, \xi\right)-k_{1}\left(z, x^{\prime}, x, \xi\right)\right)
$$

Estimating the partial differentials of $\varphi_{\left(z^{\prime}, z\right)}$ w.r.t. $x^{\prime}, x$ and $\xi$ we have

$$
\left|\partial_{x}^{\beta} \partial_{x^{\prime}}^{\beta^{\prime}} \partial_{\xi}^{\alpha} e^{i \varphi_{\left(z^{\prime}, z\right)}\left(x^{\prime}, x, y, \xi, \eta\right)}\right| \leq C\langle\xi\rangle^{\left|\beta^{\prime}\right|}\langle\xi-\eta ; \xi\rangle^{|\beta|}\langle x-y\rangle^{|\alpha|+\left|\beta^{\prime}\right|}
$$

Following [23, proof of Theorem 10.2.2], we find that $q_{\left(z^{\prime}, z\right)}\left(x^{\prime}, x, \xi\right)$ is in $\mathscr{C}^{\infty}$, bounded, with bounded differentials, as $\xi$ remains in a bounded domain.

We shall split $q_{\left(z^{\prime}, z\right)}$ into two pieces. Let $\chi \in \mathscr{C}_{c}^{\infty}\left(\mathbf{R}^{n}\right)$ be such that $\chi(\xi)=1$ if $|\xi| \leq \frac{1}{2}$ and $\chi(\xi)=0$ if $|\xi| \geq \frac{2}{3}$ and set $\chi_{0}(\eta, \xi):=\chi\left(\frac{\eta-\xi}{(1-\tau)\langle\xi\rangle}\right)$, with $0<\tau<1$ and $\chi_{\infty}(\eta, \xi):=1-\chi_{0}(\eta, \xi)$. We then write $q_{\left(z^{\prime}, z\right)}=q_{\left(z^{\prime}, z\right), \infty}+q_{\left(z^{\prime}, z\right), 0}$ where

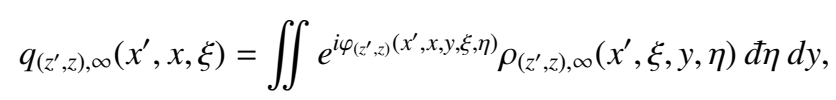

with $\rho_{\left(z^{\prime}, z\right), \infty}\left(x^{\prime}, \xi, y, \eta\right)=\rho_{\left(z^{\prime}, z\right)}\left(x^{\prime}, \xi, y, \eta\right) \chi_{\infty}(\eta, \xi)$. On the support of $\chi_{\infty}(\eta, \xi)$ we have

$$
|\eta-\xi| \geq \frac{1}{2}(1-\tau)\langle\xi\rangle
$$

We find $\left\langle\nabla_{\eta} \varphi_{\left(z^{\prime}, z\right)}\left(x^{\prime}, x, y, \xi, \eta\right)\right\rangle=\langle x-y\rangle$ and have

$$
\nabla_{y} \varphi_{\left(z^{\prime}, z\right)}\left(x^{\prime}, x, y, \xi, \eta\right)=-(\xi-\eta)+\Delta \nabla_{y} k_{1}\left(z, x^{\prime}, y, \xi\right) .
$$

For $\Delta$ sufficiently small, we find

$$
\left|\nabla_{y} \varphi_{\left(z^{\prime}, z\right)}\left(x^{\prime}, x, y, \xi, \eta\right)\right| \geq C|\xi-\eta| \geq C^{\prime}\langle\xi\rangle
$$

from (A.3). Then, setting

$$
T_{0}=\left\langle\nabla_{\eta} \varphi\right\rangle^{-2}\left(1-i\left\langle\nabla_{\eta} \varphi \mid \nabla_{\eta}\right\rangle\right), \quad T_{1}=-i\left|\nabla_{y} \varphi\right|^{-2}\left\langle\nabla_{y} \varphi \mid \nabla_{y}\right\rangle,
$$

we write, for $l$ sufficiently large,

$$
q_{\left(z^{\prime}, z\right), \infty}\left(x^{\prime}, x, \xi\right)=\iint e^{i \varphi_{\left(z^{\prime}, z\right)}\left(x^{\prime}, x, y, \xi, \eta\right)}\left(T_{0}^{t}\right)^{l}\left(T_{1}^{t}\right)^{l} \rho_{\left(z^{\prime}, z\right), \infty}\left(x^{\prime}, \xi, y, \eta\right) d \eta d y .
$$

The estimates above allow to conclude that $q_{\left(z^{\prime}, z\right), \infty}\left(x^{\prime}, x, \xi\right)$ is bounded w.r.t. $z^{\prime}$ and $z$ with values in $S^{-\infty}\left(X^{\prime} \times\right.$ $\left.X \times \mathbf{R}^{n}\right)$.

Next, we set $\rho_{\left(z^{\prime}, z\right), 0}\left(x^{\prime}, \xi, y, \eta\right)=\rho_{\left(z^{\prime}, z\right)}\left(x^{\prime}, \xi, y, \eta\right) \chi_{0}(\eta, \xi)$ and write

$$
k_{1}\left(z, x^{\prime}, y, \xi\right)-k_{1}\left(z, x^{\prime}, x, \xi\right)=\left\langle y-x \mid \tilde{k}_{1}\left(z, x^{\prime}, y, x, \xi\right)\right\rangle,
$$

by Taylor's formula. Note that $\tilde{k}_{1}\left(z, x^{\prime}, y, x, \xi\right)$ is then bounded w.r.t. $z$ with values in $S^{1}\left(X^{\prime} \times Y \times X \times \mathbf{R}^{n}\right)$. Observe that $|\xi-\eta| \leq \frac{2}{3}(1-\tau)\langle\xi\rangle$ on the support of $\chi_{0}(\eta, \xi)$ and thus for $1-\tau$ sufficiently small we have

$$
C\langle\eta\rangle \leq\langle\xi\rangle \leq C^{\prime}\langle\eta\rangle
$$


The change of variable $\eta \rightarrow \eta+\Delta \tilde{k}_{1}\left(z, x^{\prime}, y, x, \xi\right)$ is then global for $\Delta$ sufficiently small. We obtain

$$
q_{\left(z^{\prime}, z\right), 0}\left(x^{\prime}, x, \xi\right)=\iint e^{i\langle x-y \mid \xi-\eta\rangle} \rho_{\left(z^{\prime}, z\right), 0}\left(x^{\prime}, \xi, y, \eta-\Delta \tilde{k}_{1}\left(z, x^{\prime}, y, x, \xi\right)\right) d \eta d y .
$$

For $\Delta$ sufficiently small, we have

$$
C\langle\eta\rangle \leq \eta-\Delta \tilde{k}_{1}\left(z, x^{\prime}, y, x, \xi\right) \leq C^{\prime}\langle\eta\rangle, \quad \text { if }|\xi| \geq 1,
$$

on the support of the integrand after the change of variable. As in the proof of Lemma 1.7, these estimates allow to show that $\rho_{\left(z^{\prime}, z\right), 0}^{\prime}\left(x, x^{\prime}, \xi, y, \eta\right)=\rho_{\left(z^{\prime}, z\right), 0}\left(x^{\prime}, \xi, y, \eta-\Delta \tilde{k}_{1}\left(z, x^{\prime}, y, x, \xi\right)\right)$ is bounded w.r.t. $z^{\prime}$ and $z$ with values in $S_{\tilde{\rho}, \tilde{\delta}}^{m, m^{\prime}}\left(\left(X \times X^{\prime}\right) \times \mathbf{R}^{n} \times Y \times \mathbf{R}^{n}\right)$, space of multiple symbols. By Theorem 2.2.5 in [23] and Remark 1 that follows (see also [21]), we see that $q_{\left(z^{\prime}, z\right), 0,0}\left(x^{\prime}, x, \xi\right)$ is bounded w.r.t. $z^{\prime}$ and $z$ with values in $S_{\tilde{\rho}, \tilde{\delta}}^{m+m^{\prime}}\left(X^{\prime} \times X \times \mathbf{R}^{n}\right)$.

\section{A.3 Proof of Lemma 1.13}

Let $\chi \in \mathscr{C}_{c}^{\infty}\left(\mathbf{R}^{n}\right)$ be such that $\chi(\xi)=1$ if $|\xi| \leq \frac{1}{2}$ and $\chi(\xi)=0$ if $|\xi| \geq \frac{2}{3}$ and set $\chi_{\infty}(\eta, \xi):=1-\chi\left(\frac{\eta-\xi}{(1-\tau)\langle\xi\rangle}\right)$, with $0<\tau<1$. we write $\mathcal{F}=\mathcal{F}_{\infty}+\mathcal{F}_{0}$ with

$$
\mathcal{F}_{\infty}(u)\left(x^{\prime}\right):=\iint e^{i\left\langle x^{\prime}-x \mid \xi\right\rangle} f_{\left(z^{\prime}, z\right), \infty}\left(x^{\prime}, x, \xi\right) u(x) d \xi d x,
$$

where

$$
f_{\left(z^{\prime}, z\right), \infty}\left(x^{\prime}, x, \xi\right):=\iint e^{i\langle y-x \mid \eta-\xi\rangle} e^{i \Delta\left(k_{1}\left(z, x^{\prime}, y, \xi\right)-k_{1}(z, x, y, \eta)\right)} \chi_{\infty}(\eta, \xi) \underline{\sigma}_{\left(z^{\prime}, z\right)}\left(x^{\prime}, y, \xi\right) \underline{\sigma}_{\left(z^{\prime}, z\right)}(x, y, \eta) d \eta d y .
$$

with $\underline{\sigma}_{\left(z^{\prime}, z\right)}=\sigma_{\left(z^{\prime}, z\right)} e^{-\Delta l_{1}(z, x, y, \xi)}$. Thus, the amplitude $\underline{\sigma}_{\left(z^{\prime}, z\right)}(x, y, \xi)$ is bounded w.r.t. $\Delta$ and $z$ with values in $S_{\min \left(\rho, \rho^{\prime}\right)}^{m}\left(X \times Y \times \mathbf{R}^{n}\right)$.

We set

$$
\varphi\left(x^{\prime}, y, x, \xi, \eta\right):=\langle y-x \mid \eta-\xi\rangle+\Delta\left(k_{1}\left(z, x^{\prime}, y, \xi\right)-k_{1}(z, x, y, \eta)\right)
$$

We observe that

$$
\left|\nabla_{x^{\prime}} \varphi\right| \leq C\langle\xi\rangle, \quad\left|\nabla_{x} \varphi\right| \leq C\langle\eta-\xi ; \xi\rangle, \quad\left|\nabla_{\xi} \varphi\right| \leq C\langle y-x\rangle,
$$

and we thus find

$$
\left|\partial_{x^{\prime}}^{\beta^{\prime}} \partial_{x}^{\beta} \partial_{\xi}^{\alpha} e^{i \varphi\left(x^{\prime}, y, x, \xi, \eta\right)}\right| \leq C\langle\xi\rangle^{\left|\beta^{\prime}\right|}\langle\eta-\xi ; \xi\rangle^{|\beta|}\langle y-x\rangle^{|\alpha|} .
$$

Computing the partial differentials of $\varphi$ w.r.t. $y$ and $\eta$ we find

$$
\begin{aligned}
& \nabla_{\eta} \varphi\left(x^{\prime}, y, x, \xi, \eta\right)=y-x-\Delta \nabla_{\eta} k_{1}(z, x, y, \eta) \\
& \nabla_{y} \varphi\left(x^{\prime}, y, x, \xi, \eta\right)=\eta-\xi+\Delta\left(\nabla_{y} k_{1}\left(z, x^{\prime}, y, \xi\right)-\nabla_{y} k_{1}(z, x, y, \eta)\right)
\end{aligned}
$$

We have

$$
\left\langle\nabla_{\eta} \varphi\left(x^{\prime}, y, x, \xi, \eta\right)\right\rangle \geq C\langle y-x\rangle .
$$

To estimate $\left|\nabla_{y} \varphi\right|$ from below we write

$$
\nabla_{y} \varphi\left(x^{\prime}, y, x, \xi, \eta\right)=\eta-\xi+\Delta\left(\nabla_{y} k_{1}(z, x, y, \xi)-\nabla_{y} k_{1}(z, x, y, \eta)\right)+\Delta\left(\nabla_{y} k_{1}\left(z, x^{\prime}, y, \xi\right)-\nabla_{y} k_{1}(z, x, y, \xi)\right),
$$

and with Taylor's formula we have, for $\Delta$ sufficiently small,

$$
\left|\eta-\xi+\Delta\left(\nabla_{y} k_{1}(z, x, y, \xi)-\nabla_{y} k_{1}(z, x, y, \eta)\right)\right| \geq C|\eta-\xi| .
$$


We also have

$$
\left|\Delta\left(\nabla_{y} k_{1}\left(z, x^{\prime}, y, \xi\right)-\nabla_{y} k_{1}(z, x, y, \xi)\right)\right| \leq \Delta C\langle\xi\rangle .
$$

Thus, for $\Delta$ sufficiently small, on the support of $\chi_{\infty}(\eta, \xi)$, where $|\eta-\xi| \geq \frac{1}{2}(1-\tau)\langle\xi\rangle$, the previous estimates yield

$$
\left|\nabla_{y} \varphi\left(x^{\prime}, y, x, \xi, \eta\right)\right| \geq C|\eta-\xi| \geq C^{\prime}\langle\xi\rangle .
$$

Introducing

$$
T_{0}=\left\langle\nabla_{\eta} \varphi\right\rangle^{-2}\left(1-i\left\langle\nabla_{\eta} \varphi \mid \nabla_{\eta}\right\rangle\right), \quad T_{1}=-i\left|\nabla_{y} \varphi\right|^{-2}\left\langle\nabla_{y} \varphi \mid \nabla_{y}\right\rangle,
$$

by integration by parts we obtain

$$
f_{\left(z^{\prime}, z\right), \infty}\left(x^{\prime}, x, \xi\right):=\iint e^{i\langle y-x \mid \eta-\xi\rangle} e^{i \Delta\left(k_{1}\left(z, x^{\prime}, y, \xi\right)-k_{1}(z, x, y, \eta)\right)}\left(T_{0}^{t}\right)^{l}\left(T_{1}^{t}\right)^{l}\left(\chi_{\infty}(\eta, \xi) \underline{\sigma}_{\left(z^{\prime}, z\right)}\left(x^{\prime}, y, \xi\right) \bar{\sigma}_{\left(z^{\prime}, z\right)}(x, y, \eta)\right) d \eta d y
$$

for an arbitrary large $l \in \mathbf{N}$. Because of estimates (A.4), (A.5), and (A.6), we find that $f_{\left(z^{\prime}, z\right), \infty}$ is bounded w.r.t. $z^{\prime}$ and $z$ with values in $S^{-\infty}\left(X^{\prime} \times X \times \mathbf{R}^{n}\right)$. In particular, for a seminorm $p$ on $S_{\min \left(\rho, \rho^{\prime}\right)}^{0}$, there exists a seminorm $q$ on $S_{\rho}^{0}$ such that

$$
p\left(f_{\left(z^{\prime}, z\right), \infty}\right) \leq C q\left(\sigma_{\left(z^{\prime}, z\right)}\right)^{2} .
$$

We now consider the remaining part of the operator, $\mathcal{F}_{0}$. We set $\chi_{0}(\eta, \xi)=\chi\left(\frac{\eta-\xi}{(1-\tau)\langle\xi\rangle}\right)=1-\chi_{\infty}(\eta, \xi)$. Observe that on the support of $\chi_{0}(\eta, \xi)$, for $1-\tau$ small, we have

$$
C\langle\xi\rangle \leq\langle\eta\rangle \leq C^{\prime}\langle\xi\rangle
$$

With Taylor's formula we write

$$
\begin{aligned}
k_{1}\left(z, x^{\prime}, y, \xi\right)-k_{1}(z, x, y, \eta)= & \int_{0}^{1}\left(\left\langle x^{\prime}-x \mid \partial_{2} k_{1}\left(z, x+s\left(x^{\prime}-x\right), y, \eta+s(\xi-\eta)\right)\right\rangle\right. \\
& \left.+\left\langle\xi-\eta \mid \partial_{4} k_{1}\left(z, x+s\left(x^{\prime}-x\right), y, \eta+s(\xi-\eta)\right)\right\rangle\right) d s \\
= & \left\langle x^{\prime}-x \mid \tilde{k}_{1}\left(z, x^{\prime}, y, x, \xi, \eta\right)\right\rangle+\left\langle\xi-\eta \mid \tilde{\tilde{k}}_{1}\left(z, x^{\prime}, y, x, \xi, \eta\right)\right\rangle .
\end{aligned}
$$

By Theorem 1.1.9 in [16], and (A.7) we observe that, on the support of $\chi_{0}, \tilde{k}_{1}$ satisfies the estimates of symbols in $S^{1}$ w.r.t. the variables $x^{\prime}, y, x, \eta$ (or equivalently w.r.t. $x^{\prime}, y, x, \xi$ ), uniformly w.r.t. $z$. It is also homogeneous of degree one in $(\xi, \eta)$, for $|\eta|$ large.

Similarly we can consider $\tilde{\tilde{k}}_{1}$ bounded w.r.t. to $z$ and satisfies the estimates of symbols in $S^{0}$, w.r.t. the variables $x^{\prime}, y, x, \eta$, and homogeneous of degree zero in $(\xi, \eta)$, for $|\eta|$ large. We thus obtain

$$
\begin{aligned}
\mathcal{F}_{0}(u)\left(x^{\prime}\right)=\iint e^{i\left\langle x^{\prime}-x \mid \xi\right\rangle}\left(\iint e^{i\langle y-x \mid \eta-\xi\rangle} \rho_{\left(z^{\prime}, z\right)}\left(x^{\prime}, y, x, \xi, \eta\right)\right. & \\
& \left.\times e^{i \Delta\left\langle x^{\prime}-x \mid \tilde{k}_{1}\left(z, x^{\prime}, y, x, \xi, \eta\right)\right\rangle} e^{\left.\left.i \Delta\langle\xi-\eta| \tilde{k}_{1}\left(z, x^{\prime}, y, x, \xi, \eta\right)\right)\right\rangle} d \eta d y\right) u(x) d \xi d x,
\end{aligned}
$$

where

$$
\rho_{\left(z^{\prime}, z\right)}\left(x^{\prime}, y, x, \xi, \eta\right)=\chi_{0}(\eta, \xi) \underline{\sigma}_{\left(z^{\prime}, z\right)}\left(x^{\prime}, y, \xi\right) \underline{\sigma}_{\left(z^{\prime}, z\right)}(x, y, \eta) .
$$

The change of variables

$$
(\xi, \eta) \rightarrow\left(\xi+\Delta \tilde{k}_{1}\left(z, x^{\prime}, y, x, \xi, \eta\right), \eta+\Delta \tilde{k}_{1}\left(z, x^{\prime}, y, x, \xi, \eta\right)\right)=H_{\left(\Delta, z, x^{\prime}, y, x\right)}(\xi, \eta)
$$

is a global diffeomorphism for $\Delta$ small enough (uniformly in $z^{\prime}$ and $z$ in $[0, Z]$ ). We denote

$$
(\tilde{\xi}, \tilde{\eta})\left(\Delta, z, x^{\prime}, y, x, \xi, \eta\right)=H_{\left(\Delta, z, x^{\prime}, y, x\right)}^{-1}(\xi, \eta),
$$


which, for $\Delta$ sufficiently small, and $|\eta|$ sufficiently large, is homogeneous of degree one in $(\xi, \eta)$, continuous w.r.t. $z$, and smooth w.r.t. $\Delta$ and satisfies the estimates of symbols in $S^{1}$ w.r.t. to the variables $x^{\prime}, y, x, \eta$ (or equivalently w.r.t. $x^{\prime}, y, x, \xi$ ) by Lemma 2.24 in [29] and (A.7). We thus have

$$
\begin{aligned}
\mathcal{F}_{0}(u)\left(x^{\prime}\right)=\iint e^{i\left\langle x^{\prime}-x \mid \xi\right\rangle} & \left(\iint e^{i\langle y-x \mid \eta-\xi\rangle} e^{i \Delta\left\langle\xi-\eta \mid \tilde{k}_{1}\left(z, x^{\prime}, y, x,(\tilde{\xi}, \tilde{\eta})\left(\Delta, z, x^{\prime}, y, x, \xi, \eta\right)\right)\right\rangle}\right. \\
& \left.\times \rho_{\left(z^{\prime}, z\right)}\left(x^{\prime}, y, x,(\tilde{\xi}, \tilde{\eta})\left(\Delta, z, x^{\prime}, y, x, \xi, \eta\right)\right) \mathcal{J}_{1}\left(\Delta, z, x^{\prime}, y, x, \xi, \eta\right) d \eta d y\right) u(x) d \xi d x .
\end{aligned}
$$

where $\mathcal{J}_{1}\left(\Delta, z, x^{\prime}, y, \xi, \eta\right)$ is the Jacobian, which is homogeneous of degree zero in $(\xi, \eta)$, and is continuous w.r.t. $z$ and $\mathscr{C}^{\infty}$ w.r.t. $\Delta$ with values in the space of multiple symbols $S^{0,0}\left(\left(X^{\prime} \times X\right) \times \mathbf{R}^{n} \times Y \times \mathbf{R}^{n}\right)$ because of (A.7). For the sake of concision, we denote $\tilde{\xi}\left(\Delta, z, x^{\prime}, y, x, \xi, \eta\right)$ simply by $\tilde{\xi}$, with a similar notation for $\tilde{\eta}$.

Note that for $\Delta$ sufficiently small, (A.7) also holds for the new variables. By Lemma 1.7 and (A.7), $\underline{\sigma}_{\left(z^{\prime}, z\right)}\left(x^{\prime}, y, \tilde{\xi}\right)$ and $\underline{\sigma}_{\left(z^{\prime}, z\right)}(x, y, \tilde{\eta})$ are bounded w.r.t. $\Delta$ and $z$ with values in $S_{\min \left(\rho, \rho^{\prime}\right)}^{0,0}\left(\left(X^{\prime} \times X\right) \times \mathbf{R}^{n} \times Y \times \mathbf{R}^{n}\right)$, for $\Delta$ sufficiently small. A similar observation holds for $\chi_{0}(\tilde{\eta}, \tilde{\xi})$. Because of (A.7), $\tilde{\tilde{k}}_{1}\left(z, x^{\prime}, y, x, \tilde{\xi}, \tilde{\eta}\right)$ satisfies the same properties as $\tilde{\tilde{k}}_{1}\left(z, x^{\prime}, y, x, \xi, \eta\right)$ listed above.

The change of variables $y \rightarrow y-\Delta \tilde{\tilde{k}}_{1}\left(z, x^{\prime}, y, x, \tilde{\xi}, \tilde{\eta}\right)=K_{\left(\Delta, z, x^{\prime}, x, \xi, \eta\right)}(y)$ is also a global diffeomorphism for $\Delta$ small enough (uniformly in $z^{\prime}$ and $z$ in $[0, Z]$ ). We set $\tilde{y}\left(\Delta, z, x^{\prime}, y, x, \xi, \eta\right)=K_{\left(\Delta, z, x^{\prime}, x, \xi, \eta\right)}^{-1}(y)$. The function $\tilde{y}\left(\Delta, z, x^{\prime}, y, x, \xi, \eta\right)$ is homogeneous of degree zero in $(\xi, \eta)$, for $|\eta|$ sufficiently large, continuous w.r.t. $z$, and smooth w.r.t. $\Delta$ and satisfies the estimates of symbols in $S^{0}$ w.r.t. to the variables $x^{\prime}, y, x, \eta$ (or equivalently w.r.t. $x^{\prime}, y, x, \xi$ ) because of (A.7). We obtain

$$
\begin{aligned}
& \mathcal{F}(u)\left(x^{\prime}\right)=\iint e^{i\left\langle x^{\prime}-x \mid \xi\right\rangle} u(x)\left(\iint e^{i\langle y-x \mid \eta-\xi\rangle} \rho_{\left(z^{\prime}, z\right)}\left(x^{\prime}, \tilde{y}, x, \tilde{\xi}, \tilde{\eta}\right)\right. \\
&\left.\times \mathcal{J}_{1}\left(\Delta, z, x^{\prime}, \tilde{y}, x, \xi, \eta\right) \mathcal{J}_{2}\left(\Delta, z, x^{\prime}, y, x, \xi, \eta\right) d \eta d y\right) d \xi d x,
\end{aligned}
$$

where $\mathcal{J}_{2}\left(\Delta, z, x^{\prime}, y, x, \xi, \eta\right)$ is the Jacobian, which is homogeneous of degree zero in $(\xi, \eta)$, for $|\eta|$ sufficiently large, and is continuous w.r.t. $z$ and $\mathscr{C}^{\infty}$ w.r.t. $\Delta$ with values in $S^{0,0}\left(\left(X^{\prime} \times X\right) \times \mathbf{R}^{n} \times Y \times \mathbf{R}^{n}\right)$. We also see that symbol types and homogeneities of the terms in the integrals are preserved through this change of variable. Note that we modify the form of $\tilde{\xi}$ and $\tilde{\eta}$ according to the second change of variable. We do not write it explicitly for concision.

Setting

$$
q_{\left(z^{\prime}, z\right)}\left(x^{\prime}, x, y, \xi, \eta\right)=\chi_{0}(\tilde{\eta}, \tilde{\xi}) \underline{\sigma}_{\left(z^{\prime}, z\right)}\left(x^{\prime}, \tilde{y}, \tilde{\xi}\right) \underline{\sigma}_{\left(z^{\prime}, z\right)}(x, \tilde{y}, \tilde{\eta}) \mathcal{J}_{1}\left(\Delta, z, x^{\prime}, \tilde{y}, x, \xi, \eta\right) \mathcal{J}_{2}\left(\Delta, z, x^{\prime}, y, x, \xi, \eta\right),
$$

with the same argumentation as above, because of $(A .7), q_{\left(z^{\prime}, z\right)}$ is bounded w.r.t. $z^{\prime}$ and $z$ with values in the space of multiple symbols $S_{\min \left(\rho, \rho^{\prime}\right)}^{0,0}\left(\left(X^{\prime} \times X\right) \times \mathbf{R}^{n} \times Y \times \mathbf{R}^{n}\right)$. We have

$$
\mathcal{F}_{0}(u)\left(x^{\prime}\right)=\iint e^{i\left\langle x^{\prime}-x \mid \xi\right\rangle} f_{\left(z^{\prime}, z\right), 0}\left(x^{\prime}, x, \xi\right) u(x) d \xi d x
$$

with

$$
f_{\left(z^{\prime}, z\right), 0}\left(x^{\prime}, x, \xi\right)=\iint e^{i\langle y-x \mid \eta-\xi\rangle} q\left(x^{\prime}, y, x, \xi, \eta\right) d \eta d y=\left.e^{-i\left\langle D_{y} \mid D_{\eta}\right\rangle} q\left(x^{\prime}, y, x, \xi, \eta\right)\right|_{y=x, \eta=\xi} .
$$

From Theorem 2.2.5 in [23] and Remark 1 that follows (see also [21]), we obtain that $f_{\left(z^{\prime}, z\right), 0}\left(x^{\prime}, x, \xi\right)$ is bounded w.r.t. $z^{\prime}$ and $z$ with values in $S_{\min \left(\rho, \rho^{\prime}\right)}^{0}\left(X^{\prime} \times X \times \mathbf{R}^{n}\right)$.

With the form obtained for $f_{\left(z^{\prime}, z\right), 0}$, from the continuity of the composition-like formula we obtain the second part of the statement.

\section{A.4 Proof of Theorem 2.20: sharp Gårding inequality}

We follow the proof of the sharp Gårding inequality as given in [23, Section 3.4] and [39, Chapter VII]. We introduce the following function

$$
F(\xi, \zeta)=\langle\xi\rangle^{-n / 4} q\left(\langle\xi\rangle^{-\frac{1}{2}}(\zeta-\xi)\right)
$$


where $q(\sigma)$ is real, even, belongs to $\mathscr{C}_{c}^{\infty}(|\sigma| \leq 1)$ and satisfies $\int q(\sigma)^{2} d \sigma=1$ and we set

$$
\mu(\zeta, x, \xi)=\int F(\zeta, \eta) p(x, \eta) F(\xi, \eta) d \eta,
$$

which is the so-called Friedrichs' symmetrization of the symbol $p$. By Theorem 3.4.2 in [23], since $p(x, \xi) \in$ $\mathcal{M}_{k} S_{\rho}^{1}\left(X \times \mathbf{R}^{n}\right)$, the function $\mu(\zeta, x, \xi)$ is a double symbol that belongs to $\mathcal{M}_{k} S_{\frac{1}{2}, \delta}^{1,0}\left(\mathbf{R}^{n} \times X \times \mathbf{R}^{n}\right)$ (Note that since we are not interested in an asymptotic formula for $\mu(\zeta, y, \xi)$ the first part of the proof of Theorem 3.4.2 in [23] applies to the case $\left.\rho=\delta=\frac{1}{2}\right)$. Then we have $\mu\left(D_{x}, y, D_{y}\right)=v\left(x, D_{x}\right)$, with the symbol $v(x, \xi) \in$ $\mathcal{M}_{k} S_{\frac{1}{2}, \delta}^{1}\left(X \times \mathbf{R}^{n}\right)$ given by [23, Theorem 2.2.5]

$$
v(x, \xi)=\iint e^{-i\langle y \mid \xi-\zeta\rangle} \mu(\zeta, x-y, \xi) d y d \zeta,
$$

as an oscillatory integral. The operator $v\left(x, D_{x}\right)$ is non-negative as an operator, i.e., for $u \in\left(\mathscr{P}\left(\mathbf{R}^{n}\right)\right)^{k}$ we have $\operatorname{Re}\left(v\left(x, D_{x}\right) u, u\right)_{\left(\left(L^{2}\right)^{k},\left(L^{2}\right)^{k}\right)} \geq 0[23$, Theorem 3.4.3].

Next, we study the properties of $(v-p)(x, \xi)$. We set

$$
\begin{aligned}
v_{0}(x, \xi) & =\mu(\xi, x, \xi)=\int F(\xi, \eta)^{2} p(x, \eta) d \eta=\int p\left(x, \xi+\sigma\langle\xi\rangle^{\frac{1}{2}}\right) q(\sigma)^{2} d \sigma \\
& =p(x, \xi)+\iint_{0}^{1}(1-s) \partial_{\xi}^{2} p\left(x, \xi+s \sigma\langle\xi\rangle^{\frac{1}{2}} ; \sigma\langle\xi\rangle^{\frac{1}{2}}, \sigma\langle\xi\rangle^{\frac{1}{2}}\right) d s q(\sigma)^{2} d \sigma,
\end{aligned}
$$

by the second-order Taylor formula using that $\int q(\sigma)^{2} d \sigma=1$ and that $q$ is even. We observe that $|\sigma| \leq 1$ on the support of the function $q(\sigma)$ which gives

$$
C\langle\xi\rangle \leq\left\langle\xi+\langle\xi\rangle^{\frac{1}{2}} \sigma\right\rangle \leq C^{\prime}\langle\xi\rangle .
$$

From (2.9) we thus obtain that $v_{0}(x, \xi)-p(x, \xi) \in \mathcal{M}_{k} S_{\rho}^{0}\left(X \times \mathbf{R}^{n}\right)$. We now prove the following lemma.

Lemma A.2. The symbol $\left(v-v_{0}\right)(x, \xi)$ belongs to $\mathcal{M}_{k} S_{\frac{1}{2}, \delta}^{0}\left(X \times \mathbf{R}^{n}\right)$.

Proof. We first define

$$
v_{1}(x, \xi)=-\left.i \sum_{j} \partial_{x_{j}} \partial_{\zeta_{j}} \mu(\zeta, x, \xi)\right|_{\zeta=\xi}=-i \sum_{j} \int \partial_{\xi_{j}} F(\xi, \eta) \partial_{x_{j}} p(x, \eta) F(\xi, \eta) d \eta
$$

and prove that $v_{1}(x, \xi) \in \mathcal{M}_{k} S_{\rho}^{0}\left(X \times \mathbf{R}^{n}\right)$. From [23, Lemma 3.4.1] $\partial_{\xi_{j}} F(\xi, \eta)$ has the form

$$
\partial_{\xi_{j}} F(\xi, \eta)=\langle\xi\rangle^{-n / 4} \sum_{|\gamma| \leq 1, \gamma_{1} \leq \gamma} \psi_{\gamma, \gamma_{1}}(\xi)\left((\eta-\xi)\langle\xi\rangle^{-\frac{1}{2}}\right)^{\gamma_{1}}\left(\partial_{\sigma}^{\gamma} q\right)\left((\eta-\xi)\langle\xi\rangle^{-\frac{1}{2}}\right),
$$

where $\psi_{\gamma, \gamma_{1}} \in S_{1,0}^{-1+\frac{1}{2}\left|\gamma-\gamma_{1}\right|}\left(\mathbf{R}^{n}\right)$. From the definition of $F$ in (A.8) we write the $j$ th term in the sum in (A.10) as

$$
\begin{aligned}
v_{1}^{(j)}(x, \xi)= & -i\langle\xi\rangle^{-n / 2} \sum_{|\gamma| \leq 1, \gamma_{1} \leq \gamma} \psi_{\gamma, \gamma_{1}}(\xi) \int\left((\eta-\xi)\langle\xi\rangle^{-\frac{1}{2}}\right)^{\gamma_{1}}\left(\partial_{\sigma}^{\gamma} q\right)\left((\eta-\xi)\langle\xi\rangle^{-\frac{1}{2}}\right) \\
& \times \partial_{x_{j}} p(x, \eta) q\left((\eta-\xi)\langle\xi\rangle^{-\frac{1}{2}}\right) d \eta \\
= & -i \sum_{|\gamma| \leq 1, \gamma_{1} \leq \gamma} \psi_{\gamma, \gamma_{1}}(\xi) \int \sigma^{\gamma_{1}}\left(\partial_{\sigma}^{\gamma} q\right)(\sigma) q(\sigma) \partial_{x_{j}} p\left(x, \xi+\langle\xi\rangle^{\frac{1}{2}} \sigma\right) d \sigma,
\end{aligned}
$$

after a change of variable. There are two cases to consider in the sum in (A.11): a) $\gamma_{1}=\gamma$ and b) $\gamma_{1}=0$ and $|\gamma|=1$. From (A.9), and (2.8) and from the fact that $\psi_{\gamma, \gamma} \in S_{1,0}^{-1}\left(\mathbf{R}^{n}\right)$ we find that the contribution from case $a$ ), i.e.,

$$
v_{1}^{(j, a)}(x, \xi)=-i \sum_{|\gamma| \leq 1} \psi_{\gamma, \gamma}(\xi) \int \sigma^{\gamma}\left(\partial_{\sigma}^{\gamma} q\right)(\sigma) q(\sigma) \partial_{x_{j}} p\left(x, \xi+\langle\xi\rangle^{\frac{1}{2}} \sigma\right) d \sigma
$$


satisfies $\left|v_{1}^{(j, a)}(x, \xi)\right| \leq C$. Computing $\partial_{x}^{\alpha} \partial_{\xi}^{\beta} v_{1}^{(j, a)}(x, \xi)$ we find it to be a linear combination of terms of the form

$$
\sum_{|\gamma| \leq 1} \partial_{\xi}^{\beta_{1}} \psi_{\gamma, \gamma}(\xi) \int \sigma^{\gamma}\left(\partial_{\sigma}^{\gamma} q\right)(\sigma) q(\sigma) \partial_{x}^{\alpha} \partial_{\xi}^{\beta_{2}} \partial_{x_{j}} p\left(x, \xi+\langle\xi\rangle^{\frac{1}{2}} \sigma\right) d \sigma, \quad \text { with } \beta_{1}+\beta_{2}=\beta
$$

From (A.9) and (2.9) we see that $\partial_{x}^{\alpha} \partial_{\xi}^{\beta_{2}} \partial_{x_{j}} p\left(x, \xi+\langle\xi\rangle^{\frac{1}{2}} \sigma\right)$ is in $\mathcal{M}_{k} S_{\rho}^{\delta|\alpha|+\rho\left(1-\left|\beta_{2}\right|\right)}\left(X \times \mathbf{R}^{n}\right)$ uniformly w.r.t. $\sigma$, $|\sigma| \leq 1$ and $\partial_{\xi}^{\beta_{1}} \psi_{\gamma, \gamma}(\xi)$ is in $S_{1,0}^{-1-\left|\beta_{1}\right|}\left(X \times \mathbf{R}^{n}\right)$. As a result, $v_{1}^{(j, a)}(x, \xi)$ belongs to $\mathcal{M}_{k} S_{\rho}^{0}\left(X \times \mathbf{R}^{n}\right)$. We now consider the contribution from case b) in the sum in (A.11), i.e.,

$$
v_{1}^{(j, b)}(x, \xi)=-i \sum_{|\gamma|=1} \psi_{\gamma, 0}(\xi) \int\left(\partial_{\sigma}^{\gamma} q\right)(\sigma) q(\sigma) \partial_{x_{j}} p\left(x, \xi+\langle\xi\rangle^{\frac{1}{2}} \sigma\right) d \sigma .
$$

We write

$$
\partial_{x_{j}} p\left(x, \xi+\langle\xi\rangle^{\frac{1}{2}} \sigma\right)=\partial_{x_{j}} p(x, \xi)+\langle\xi\rangle^{\frac{1}{2}} \sum_{l} \sigma_{l} \int_{0}^{1}\left(\partial_{\xi l} \partial_{x_{j}} p\right)\left(x, \xi+t \sigma\langle\xi\rangle^{\frac{1}{2}}\right) d t
$$

Since $\int \partial_{\sigma}^{\gamma} q(\sigma) q(\sigma) d \sigma=0$ ( $q$ is even), the first term gives no contribution and we obtain

$$
v_{1}^{(j, b)}(x, \xi)=-i\langle\xi\rangle^{\frac{1}{2}} \sum_{|\gamma|=1, l} \psi_{\gamma, 0}(\xi) \iint_{0}^{1}\left(\partial_{\sigma}^{\gamma} q\right)(\sigma) q(\sigma) \sigma_{l}\left(\partial_{\xi_{l}} \partial_{x_{j}} p\right)\left(x, \xi+t \sigma\langle\xi\rangle^{\frac{1}{2}}\right) d t d \sigma .
$$

Since by (2.9), $\partial_{\xi_{l}} \partial_{x_{j}} p(x, \xi) \in \mathcal{M}_{k} S_{\rho}^{0}\left(X \times \mathbf{R}^{n}\right)$ and $\psi_{\gamma, 0}(\xi) \in S_{1,0}^{-\frac{1}{2}}\left(\mathbf{R}^{n}\right)$ we obtain that $v_{1}^{(j, b)}(x, \xi) \in \mathcal{M}_{k} S_{\rho}^{0}(X \times$ $\mathbf{R}^{n}$ ) from (A.9).

We have thus proven that $v_{1}(x, \xi) \in \mathcal{M}_{k} S_{\rho}^{0}\left(X \times \mathbf{R}^{n}\right)$. We now compute $\left(v-v_{0}\right)(x, \xi)$.

$$
\begin{aligned}
\left(v-v_{0}\right)(x, \xi) & =\int_{3} e^{-i(y|\xi-\zeta\rangle} F(\zeta, \eta) p(x-y, \eta) F(\xi, \eta) d y d \zeta d \eta-\int F(\xi, \eta)^{2} p(x, \eta) d \eta \\
& =\int_{3} e^{-i(y|\xi-\zeta\rangle} F(\zeta, \eta)(p(x-y, \eta)-p(x, \eta)) F(\xi, \eta) d y d \zeta d \eta \\
& =\int_{0}^{1} \sum_{j} \int_{3}-y_{j} e^{-i(y|\xi-\zeta\rangle} F(\zeta, \eta) \partial_{x_{j}} p(x-s y, \eta) F(\xi, \eta) d y d \zeta d \eta d s \\
& =-i \int_{0}^{1} \sum_{j} \int_{3} e^{-i\langle y \mid \xi-\zeta\rangle} \partial_{\zeta_{j}} F(\zeta, \eta) \partial_{x_{j}} p(x-s y, \eta) F(\xi, \eta) d y d \zeta d \eta d s,
\end{aligned}
$$

after an integration by parts. Arguing similarly, computing $\left(v-v_{0}-v_{1}\right)(x, \xi)$, we obtain

$$
\begin{aligned}
& \left(v-v_{0}-v_{1}\right)(x, \xi) \\
& =-i \int_{0}^{1} \sum_{j} \int_{3} e^{-i(\langle y \mid \xi-\zeta\rangle} \partial_{\zeta_{j}} F(\zeta, \eta)\left(\partial_{x_{j}} p(x-s y, \eta)-\partial_{x_{j}} p(x, \eta)\right) F(\xi, \eta) d y d \zeta d \eta d s \\
& =-\int_{0}^{1} \int_{0}^{1} s \sum_{j, l} \int_{\circledast} e^{-i(y \mid \xi-\zeta)} \partial_{\zeta_{j}, \zeta_{l}}^{2} F(\zeta, \eta) \partial_{x_{j}, x_{l}}^{2} p\left(x-s^{\prime} s y, \eta\right) F(\xi, \eta) d y d \zeta d \eta d s d s^{\prime} \\
& =-\sigma\left\{\int_{0}^{1} \int_{0}^{1} s \sum_{j, l} \int \partial_{\zeta_{j}, \zeta_{l}}^{2} F(\zeta, \eta) \partial_{x_{j}, x_{l}}^{2} p\left(\left(1-s^{\prime} s\right) x+s^{\prime} s y, \eta\right) F(\xi, \eta) d \eta d s d s^{\prime}\right\} .
\end{aligned}
$$


Observing that

$$
\tilde{p}(x, y, \eta):=\int_{0}^{1} \int_{0}^{1} s \partial_{x_{j}, x_{l}}^{2} p\left(\left(1-s^{\prime} s\right) x+s s^{\prime} y, \eta\right) d s d s^{\prime}
$$

is in $\mathcal{M}_{k} S_{\rho}^{1}\left(X \times Y \times \mathbf{R}^{n}\right)$ by (2.9), and then following the proof of Theorem 3.4.2 in [23] we find that its Friedrichs' symmetrization,

$$
\tilde{\mu}(x, \zeta, y, \xi)=\int F(\zeta, \eta) \tilde{p}(x, y, \eta) F(\xi, \eta) d \eta,
$$

is in $\mathcal{M}_{k} S_{\frac{1}{2}, \delta}^{1,0}\left(X \times \mathbf{R}^{n} \times Y \times \mathbf{R}^{n}\right)$ and thus $\partial_{\zeta_{j}, \zeta_{l}}^{2} \tilde{\mu}(x, \zeta, y, \xi)$ is in $\mathcal{M}_{k} S_{\frac{1}{2}, \delta}^{0,0}\left(X \times \mathbf{R}^{n} \times Y \times \mathbf{R}^{n}\right)$ and finally we find $\left(v-v_{0}-v_{1}\right)(x, \xi) \in \mathcal{M}_{k} S_{\frac{1}{2}, \delta}^{0}\left(X \times \mathbf{R}^{n}\right)$ by Theorem 2.2.5 in [23]. With $v_{1}(x, \xi) \in \mathcal{M}_{k} S_{\rho}^{0}\left(X \times \mathbf{R}^{n}\right)$ as proven above, this concludes the proof.

End of the proof of Theorem 2.20. As a consequence of the previous lemma we find that $(v-p)(x, \xi) \in$ $\mathcal{M}_{k} S_{\frac{1}{2}, \delta}^{0}\left(X \times \mathbf{R}^{n}\right)$ and we have

$$
\operatorname{Re}\left(p\left(x, D_{x}\right) u, u\right)_{\left(\left(L^{2}\right)^{k},\left(L^{2}\right)^{k}\right)}=\operatorname{Re}\left(v\left(x, D_{x}\right) u, u\right)_{\left(\left(L^{2}\right)^{k},\left(L^{2}\right)^{k}\right)}+\operatorname{Re}\left((p-v)\left(x, D_{x}\right) u, u\right)_{\left(\left(L^{2}\right)^{k},\left(L^{2}\right)^{k}\right)} \geq-C\|u\|_{\left(L^{2}\right)^{k}}^{2},
$$

by the Calderón-Vaillancourt Theorem (see [23, Chapter 7, Sections 1,2] or [39, Section XIII-2]).

\section{A.5 Proof of Theorem 3.4}

Let $s \in \mathbf{R}$. At first we choose the symbols $\mu(z, x, \xi)$ and $\sigma_{\left(z^{\prime}, z\right)}(x, \xi)$ with compact support w.r.t. the variables $x$ and $\xi$ to be able to carry out some integral calculus in a simple manner. In the proof, we shall always assume that $\Delta$ is sufficiently small to apply the invoked properties and results.

We let $u \in \mathscr{P}\left(\mathbf{R}^{n}\right)$. We then have

$$
\begin{aligned}
& \mu\left(z, x, D_{x}\right) \circ \mathcal{A}_{\left(z^{\prime}, z\right)} u\left(x^{\prime \prime}\right)=\iint e^{i\left\langle x^{\prime \prime}-x^{\prime} \mid \eta\right\rangle} \mu\left(z, x^{\prime \prime}, \eta\right) \iint e^{i\left\langle x^{\prime}-x \mid \xi\right\rangle} \sigma_{\left(z^{\prime}, z\right)}\left(x^{\prime}, \xi\right) e^{-\Delta a_{1}\left(z, x^{\prime}, \xi\right)} u(x) d \xi d x d \eta d x^{\prime} \\
& \quad=\iint e^{i\left\langle x^{\prime \prime}-x \mid \xi\right\rangle}\left(\iint e^{i\left\langle x^{\prime \prime}-x^{\prime} \mid \eta-\xi\right\rangle} \mu\left(z, x^{\prime \prime}, \eta\right) \underline{\sigma}_{\left(z^{\prime}, z\right)}\left(x^{\prime}, \xi\right) e^{i \Delta b_{1}\left(z, x^{\prime}, \xi\right)} e^{-i \Delta b_{1}\left(z, x^{\prime \prime}, \xi\right)} d \eta d x^{\prime}\right) e^{i \Delta b_{1}\left(z, x^{\prime \prime}, \xi\right)} u(x) d \xi d x,
\end{aligned}
$$

where we have set $\underline{\sigma}_{\left(z^{\prime}, z\right)}\left(z, x^{\prime}, \xi\right)=\sigma_{\left(z^{\prime}, z\right)}\left(x^{\prime}, \xi\right) p_{\Delta}\left(z, x^{\prime}, \xi\right)$ (recall that the matrices $c_{1}(z,$.$) and b_{1}(z,$.$) com-$ mute). We set

$$
\tilde{q}_{\left(z^{\prime}, z\right)}\left(x^{\prime \prime}, \xi\right):=\iint e^{i\left\langle x^{\prime \prime}-x^{\prime} \mid \eta-\xi\right\rangle} \mu\left(z, x^{\prime \prime}, \eta\right) \underline{\sigma}_{\left(z^{\prime}, z\right)}\left(x^{\prime}, \xi\right) e^{i \Delta b_{1}\left(z, x^{\prime}, \xi\right)} e^{-i \Delta b_{1}\left(z, x^{\prime \prime}, \xi\right)} d \eta d x^{\prime} .
$$

Observe that $\tilde{q}_{\left(z^{\prime}, z\right)}$ may not be a symbol. Using properties of the matrix $b_{1}(z,$.$) , we write$

$$
\begin{aligned}
\tilde{q}_{\left(z^{\prime}, z\right)}\left(x^{\prime \prime}, \xi\right)=\iint e^{i\left\langle x^{\prime \prime}-x^{\prime} \mid \eta-\xi\right\rangle} \mu\left(z, x^{\prime \prime}, \eta\right)\left(\underline{\sigma}_{\left(z^{\prime}, z\right)}(.) w(z, .) e^{i \Delta d_{b}(z, .)} v(z, .)\right)\left(x^{\prime}, \xi\right) & \\
& \times\left(w(z, .) e^{-i \Delta d_{b}(z, .)} v(z, .)\right)\left(x^{\prime \prime}, \xi\right) d \eta d x^{\prime},
\end{aligned}
$$

and we proceed as in the proof of Theorem 2.22: with Taylor's formula we write

$$
v\left(z, x^{\prime}, \xi\right)=v\left(z, x^{\prime \prime}, \xi\right)+\left\langle x^{\prime}-x^{\prime \prime} \mid \tilde{v}\left(z, x^{\prime}, x^{\prime \prime}, \xi\right)\right\rangle,
$$

with $\tilde{v}\left(z, x^{\prime}, x^{\prime \prime}, \xi\right)$ bounded w.r.t. $z$ with values in $\left(\mathcal{M}_{k} S^{0}\left(X^{\prime} \times X^{\prime \prime} \times \mathbf{R}^{n}\right)\right)^{n}$. We obtain

$$
\tilde{q}_{\left(z^{\prime}, z\right)}=q_{\left(z^{\prime}, z\right)}+q_{\left(z^{\prime}, z\right)}^{b}+q_{\left(z^{\prime}, z\right)}^{c},
$$

with $q_{\left(z^{\prime}, z\right)}$ as given in (3.3) and

$$
\begin{aligned}
q_{\left(z^{\prime}, z\right)}^{b}\left(x^{\prime \prime}, \xi\right):=\iint e^{i\left\langle x^{\prime \prime}-x^{\prime} \mid \eta-\xi\right\rangle} \mu(z, & \left.x^{\prime \prime}, \eta\right) \underline{\sigma}_{\left(z^{\prime}, z\right)}\left(x^{\prime}, \xi\right) \\
& \times w\left(z, x^{\prime}, \xi\right) e^{i \Delta d_{b}\left(z, x^{\prime}, \xi\right)}\left\langle x^{\prime}-x^{\prime \prime} \mid \tilde{v}\left(z, x^{\prime}, x^{\prime \prime}, \xi\right)\right\rangle e^{-i \Delta b_{1}\left(z, x^{\prime \prime}, \xi\right)} d \eta d x^{\prime},
\end{aligned}
$$


and

$$
\begin{aligned}
q_{\left(z^{\prime}, z\right)}^{c}\left(x^{\prime \prime}, \xi\right):=-\iint e^{i\left\langle x^{\prime \prime}-x^{\prime} \mid \eta-\xi\right\rangle} \mu(z, & \left.x^{\prime \prime}, \eta\right) \underline{\sigma}_{\left(z^{\prime}, z\right)}\left(x^{\prime}, \xi\right) \\
& \times w\left(z, x^{\prime}, \xi\right) e^{i \Delta\left(d_{b}\left(z, x^{\prime}, \xi\right)-d_{b}\left(z, x^{\prime \prime}, \xi\right)\right)}\left\langle x^{\prime}-x^{\prime \prime} \mid \tilde{v}\left(z, x^{\prime}, x^{\prime \prime}, \xi\right)\right\rangle d \eta d x^{\prime}
\end{aligned}
$$

Because of the diagonal form of the term $e^{i \Delta\left(d_{b}\left(z, x^{\prime}, \xi\right)-d_{b}\left(z, x^{\prime \prime}, \xi\right)\right)}$ in (3.3), the result of Proposition 1.10 for the scalar case (see also Remark 1.11), can be applied to the first term, $q_{\left(z^{\prime}, z\right)}$. The assumptions made above on the supports of $\mu(z,$.$) and \sigma_{\left(z^{\prime}, z\right)}$ can thus be relaxed in the expression of the term $q_{\left(z^{\prime}, z\right)}$ in $(3.3)$, which is then bounded w.r.t. $z^{\prime}$ and $z$ with values in $\mathcal{M}_{k} S_{\min \left(\rho, \rho^{\prime}, \rho^{\prime \prime}\right)}^{m+m^{\prime}}\left(X \times \mathbf{R}^{n}\right)$.

We shall now focus on the remainder terms, $q_{\left(z^{\prime}, z\right)}^{b}$ and $q_{\left(z^{\prime}, z\right)}^{c}$. Note that these two terms are not symbols in general. However, we shall prove that the remaining operator, with (well-defined) kernel

$$
\int e^{i\left\langle x^{\prime \prime}-x \mid \xi\right\rangle}\left(q_{\left(z^{\prime}, z\right)}^{b}+q_{\left(z^{\prime}, z\right)}^{c}\right)\left(x^{\prime \prime}, \xi\right) e^{i \Delta b_{1}\left(z, x^{\prime \prime}, \xi\right)} d \xi
$$

can be written in the form $\Delta \mathcal{R}_{\left(z^{\prime}, z\right)}$, with $\mathcal{R}_{\left(z^{\prime}, z\right)}$ satisfying estimate (3.2) and with this regularity property still holding as we relax the assumptions made above on the supports of $\mu(z,$.$) and \sigma_{\left(z^{\prime}, z\right)}$. We shall actually obtain that the components of the matrix operator $\mathcal{R}_{\left(z^{\prime}, z\right)}$ are sums of scalar FIOs of the form studied in Section 1.

We write $\left(x_{j}^{\prime}-x_{j}^{\prime \prime}\right) e^{i\left\langle x^{\prime \prime}-x^{\prime} \mid \eta-\xi\right\rangle}=i \partial_{\eta_{j}} e^{i\left\langle x^{\prime \prime}-x^{\prime} \mid \eta-\xi\right\rangle}$. An integration by parts yields

$$
\begin{array}{r}
q_{\left(z^{\prime}, z\right)}^{b}\left(x^{\prime \prime}, \xi\right)=-i \iint e^{i\left\langle x^{\prime \prime}-x^{\prime} \mid \eta-\xi\right\rangle}\left\langle\nabla_{\eta} \mu\left(z, x^{\prime \prime}, \eta\right)\right|\left(\underline{\sigma}_{\left(z^{\prime}, z\right)}(.) w(z, .) e^{i \Delta d_{b}(z, .)}\right)\left(x^{\prime}, \xi\right) \\
\left.\times \tilde{v}\left(z, x^{\prime}, x^{\prime \prime}, \xi\right)\right\rangle e^{-i \Delta b_{1}\left(z, x^{\prime \prime}, \xi\right)} d \eta d x^{\prime} .
\end{array}
$$

We now expand the first exponential term to first order, i.e.,

$$
e^{i \Delta d_{b}\left(z, x^{\prime}, \xi\right)}=1+i \Delta d_{b}\left(z, x^{\prime}, \xi\right) \int_{0}^{1} e^{i t \Delta d_{b}\left(z, x^{\prime}, \xi\right)} d t,
$$

and we obtain

$$
q_{\left(z^{\prime}, z\right)}^{b}=q_{\left(z^{\prime}, z\right)}^{b, a}+\Delta q_{\left(z^{\prime}, z\right)}^{b, 1}
$$

with

$$
q_{\left(z^{\prime}, z\right)}^{b, a}\left(x^{\prime \prime}, \xi\right)=-i \iint e^{i\left\langle x^{\prime \prime}-x^{\prime} \mid \eta-\xi\right\rangle}\left\langle\nabla_{\eta} \mu\left(z, x^{\prime \prime}, \eta\right) \mid\left(\underline{\sigma}_{\left(z^{\prime}, z\right)}(.) w(z, .)\right)\left(x^{\prime}, \xi\right) \tilde{v}\left(z, x^{\prime}, x^{\prime \prime}, \xi\right)\right\rangle e^{-i \Delta b_{1}\left(z, x^{\prime \prime}, \xi\right)} d \eta d x^{\prime},
$$

and

$$
\begin{array}{r}
q_{\left(z^{\prime}, z\right)}^{b, 1}\left(x^{\prime \prime}, \xi\right)=\iiint e^{i\left\langle x^{\prime \prime}-x^{\prime} \mid \eta-\xi\right\rangle}\left\langle\nabla_{\eta} \mu\left(z, x^{\prime \prime}, \eta\right)\right|\left(\underline{\sigma}_{\left(z^{\prime}, z\right)}(.) w(z, .) d_{b}(z, .) e^{t i \Delta d_{b}(z, .)}\right)\left(x^{\prime}, \xi\right) \\
\left.\times \tilde{v}\left(z, x^{\prime}, x^{\prime \prime}, \xi\right)\right\rangle e^{-i \Delta b_{1}\left(z, x^{\prime \prime}, \xi\right)} d \eta d x^{\prime} d t .
\end{array}
$$

The operator $\mathcal{R}_{\left(z^{\prime}, z\right)}^{b, 1}$ associated with the latter term, $q_{\left(z^{\prime}, z\right)}^{b, 1}$, has for kernel

$$
R_{\left(z^{\prime}, z\right)}^{b, 1}\left(x^{\prime \prime}, x\right)=\iint_{0}^{1} e^{i\left\langle x^{\prime \prime}-x \mid \xi\right\rangle} \underline{q}_{\left(z^{\prime}, z\right)}^{b, 1}\left(t, x^{\prime \prime}, \xi\right) d \xi d t,
$$

with

$\underline{q}_{\left(z^{\prime}, z\right)}^{b, 1}\left(t, x^{\prime \prime}, \xi\right)=\iint e^{i\left\langle x^{\prime \prime}-x^{\prime} \mid \eta-\xi\right\rangle}\left\langle\nabla_{\eta} \mu\left(z, x^{\prime \prime}, \eta\right) \mid\left(\underline{\sigma}_{\left(z^{\prime}, z\right)}() w.(z,.) d_{b}(z,.) e^{t i \Delta d_{b}(z, .)}\right)\left(x^{\prime}, \xi\right) \times \tilde{v}\left(z, x^{\prime}, x^{\prime \prime}, \xi\right)\right\rangle d \eta d x^{\prime}$. 
Note that $\underline{q}_{\left(z^{\prime}, z\right)}^{b, 1}(t,$.$) is not a symbol, in general. However, by formula (1.3), each component of the matrix$ kernel $R_{\left(z^{\prime}, z\right)}^{b, 1}\left(x^{\prime \prime}, x\right)$ is a sum of (scalar) FIO kernels of the form

$$
\iint_{0}^{1} e^{i\left\langle x^{\prime \prime}-x \mid \xi\right\rangle+t i \Delta d_{b, l}\left(z, x^{\prime \prime}, \xi\right)} \lambda_{\left(z^{\prime}, z\right)}\left(x^{\prime \prime}, \xi\right) d \xi d t, \quad 1 \leq l \leq k,
$$

(recall that $d_{b, l}, 1 \leq l \leq k$, are the diagonal entries of the $d_{b}$ ) and with a symbol $\lambda_{\left(z^{\prime}, z\right)}\left(x^{\prime \prime}, \xi\right)$ that is bounded w.r.t. to $z^{\prime}$ and $z$ with values in $S_{\min \left(\rho, \rho^{\prime}, \rho^{\prime \prime}\right)}^{m+m^{\prime}}\left(X^{\prime \prime} \times \mathbf{R}^{n}\right)$. By Theorem 1.12, we thus obtain

$$
\left\|\mathcal{R}_{\left(z^{\prime}, z\right)}^{b, 1}\right\|_{\left(\left(H^{(s)}\right)^{k},\left(H^{\left(s-m-m^{\prime}\right)}\right)^{k}\right)} \leq K^{(1)},
$$

uniformly w.r.t. $z$ and $\Delta, \Delta$ sufficiently small. This estimate holds if we relax the assumptions made above on the supports of $\mu(z,$.$) and \sigma_{\left(z^{\prime}, z\right)}$.

Similarly, we expand to first order the remaining exponential in the term $q_{\left(z^{\prime}, z\right)}^{b, a}$ and obtain

$$
q_{\left(z^{\prime}, z\right)}^{b, a}=q_{\left(z^{\prime}, z\right)}^{b, b}+\Delta q_{\left(z^{\prime}, z\right)}^{b, 2}
$$

with

$$
q_{\left(z^{\prime}, z\right)}^{b, b}\left(x^{\prime \prime}, \xi\right)=-i \iint e^{i\left\langle x^{\prime \prime}-x^{\prime} \mid \eta-\xi\right\rangle}\left\langle\nabla_{\eta} \mu\left(z, x^{\prime \prime}, \eta\right) \mid\left(\underline{\sigma}_{\left(z^{\prime}, z\right)}(.) w(z, .)\right)\left(x^{\prime}, \xi\right) \times \tilde{v}\left(z, x^{\prime}, x^{\prime \prime}, \xi\right)\right\rangle d \eta d x^{\prime}
$$

and

$$
\begin{aligned}
q_{\left(z^{\prime}, z\right)}^{b, 2}\left(x^{\prime \prime}, \xi\right)=-\iiint e^{i\left\langle x^{\prime \prime}-x^{\prime} \mid \eta-\xi\right\rangle}\left\langle\nabla_{\eta} \mu\left(z, x^{\prime \prime}, \eta\right)\right| & \left.\left(\underline{\sigma}_{\left(z^{\prime}, z\right)}(.) w(z, .)\right)\left(x^{\prime}, \xi\right) \tilde{v}\left(z, x^{\prime}, x^{\prime \prime}, \xi\right)\right\rangle \\
& \times b_{1}\left(z, x^{\prime \prime}, \xi\right) e^{-i t \Delta b_{1}\left(z, x^{\prime \prime}, \xi\right)} d \eta d x^{\prime} d t .
\end{aligned}
$$

The operator $\mathcal{R}_{\left(z^{\prime}, z\right)}^{b, 2}$ associated with the latter term, $q_{\left(z^{\prime}, z\right)}^{b, 2}$, has for kernel

$$
R_{\left(z^{\prime}, z\right)}^{b, 2}\left(x^{\prime \prime}, x\right)=\iint_{0}^{1} e^{i\left\langle x^{\prime \prime}-x \mid \xi\right\rangle} \underline{q}_{\left(z^{\prime}, z\right)}^{b, 2}\left(x^{\prime \prime}, \xi\right) e^{i(1-t) \Delta b_{1}\left(z, x^{\prime \prime}, \xi\right)} d \xi d t,
$$

where $\underline{q}_{\left(z^{\prime}, z\right)}^{b, 2}$ is in fact a symbol, that is bounded w.r.t. to $z^{\prime}$ and $z$ with values in $S_{\min \left(\rho, \rho^{\prime}, \rho^{\prime \prime}\right)}^{m+m^{\prime}}\left(X \times \mathbf{R}^{n}\right)$. By Proposition 2.6, we have

$$
\left\|\mathcal{R}_{\left(z^{\prime}, z\right)}^{b, 2}\right\|_{\left(\left(H^{(s)}\right)^{k},\left(H^{\left(s-m-m^{\prime}\right)}\right)^{k}\right)} \leq K^{(2)}
$$

uniformly w.r.t. $z$ and $\Delta, \Delta$ sufficiently small. As above, this estimate holds if we relax the assumptions made above on the supports of $\mu(z,$.$) and \sigma_{\left(z^{\prime}, z\right)}$.

For the remaining term, $q_{\left(z^{\prime}, z\right)}^{b, b}$, which is actually a symbol, we 'undo' the integration by parts, which reads

$$
q_{\left(z^{\prime}, z\right)}^{b, b}\left(x^{\prime \prime}, \xi\right)=\iint e^{i\left\langle x^{\prime \prime}-x^{\prime} \mid \eta-\xi\right\rangle} \mu\left(z, x^{\prime \prime}, \eta\right)\left(\underline{\sigma}_{\left(z^{\prime}, z\right)}(.) w(z, .)\right)\left(x^{\prime}, \xi\right)\left\langle x^{\prime}-x^{\prime \prime} \mid \tilde{v}\left(z, x^{\prime}, x^{\prime \prime}, \xi\right)\right\rangle d \eta d x^{\prime} .
$$

We proceed similarly with the term $q_{\left(z^{\prime}, z\right)}^{c}$ in (A.13). An expansion to first order of the exponential term yields

$$
q_{\left(z^{\prime}, z\right)}^{c}=q_{\left(z^{\prime}, z\right)}^{c, a}+\Delta q_{\left(z^{\prime}, z\right)}^{c, 1}
$$

with

$$
q_{\left(z^{\prime}, z\right)}^{c, a}\left(x^{\prime \prime}, \xi\right):=-\iint e^{i\left\langle x^{\prime \prime}-x^{\prime} \mid \eta-\xi\right\rangle} \mu\left(z, x^{\prime \prime}, \eta\right)\left(\underline{\sigma}_{\left(z^{\prime}, z\right)}(.) w(z, .)\right)\left(x^{\prime}, \xi\right)\left\langle x^{\prime}-x^{\prime \prime} \mid \tilde{v}\left(z, x^{\prime}, x^{\prime \prime}, \xi\right)\right\rangle d \eta d x^{\prime}
$$


and

$$
\begin{aligned}
q_{\left(z^{\prime}, z\right)}^{c, 1}\left(x^{\prime \prime}, \xi\right):=-i \iint_{0}^{1} \iint^{i\left\langle x^{\prime \prime}-x^{\prime} \mid \eta-\xi\right\rangle} \mu\left(z, x^{\prime \prime}, \eta\right)\left(\underline{\sigma}_{\left(z^{\prime}, z\right)}(.) w(z, .)\right)\left(x^{\prime}, \xi\right) \\
\quad \times\left(d_{b}\left(z, x^{\prime}, \xi\right)-d_{b}\left(z, x^{\prime \prime}, \xi\right)\right) e^{i \Delta t\left(d_{b}\left(z, x^{\prime}, \xi\right)-d_{b}\left(z, x^{\prime \prime}, \xi\right)\right)}\left\langle x^{\prime}-x^{\prime \prime} \mid \tilde{v}\left(z, x^{\prime}, x^{\prime \prime}, \xi\right)\right\rangle d \eta d x^{\prime} d t .
\end{aligned}
$$

We observe that

$$
q_{\left(z^{\prime}, z\right)}^{c, a}+q_{\left(z^{\prime}, z\right)}^{b, b}=0
$$

An integration by parts, in the term $q_{\left(z^{\prime}, z\right)}^{c, 1}$ yields

$$
\begin{aligned}
& q_{\left(z^{\prime}, z\right)}^{c, 1}\left(x^{\prime \prime}, \xi\right):=-\int_{0}^{1} \iint e^{i\left\langle x^{\prime \prime}-x^{\prime} \mid \eta-\xi\right\rangle}\left\langle\nabla_{\eta} \mu\left(z, x^{\prime \prime}, \eta\right)\right|\left(\underline{\sigma}_{\left(z^{\prime}, z\right)}(.) w(z, .)\right)\left(x^{\prime}, \xi\right) \\
&\left.\quad \times\left(d_{b}\left(z, x^{\prime}, \xi\right)-d_{b}\left(z, x^{\prime \prime}, \xi\right)\right) e^{i t \Delta\left(d_{b}\left(z, x^{\prime}, \xi\right)-d_{b}\left(z, x^{\prime \prime}, \xi\right)\right)} \tilde{v}\left(z, x^{\prime}, x^{\prime \prime}, \xi\right)\right\rangle d \eta d x^{\prime} d t .
\end{aligned}
$$

Because of the diagonal form of the term $e^{i \Delta\left(d_{b}\left(z, x^{\prime}, \xi\right)-d_{b}\left(z, x^{\prime \prime}, \xi\right)\right)}$ in (A.15) the result of Proposition 1.10, can be applied to $q_{\left(z^{\prime}, z\right)}^{c, 1}$ (see Remark 1.11). The assumptions made above on the supports of $\mu(z,$.$) and \sigma_{\left(z^{\prime}, z\right)}$ can also be relaxed in the expression of the term $q_{\left(z^{\prime}, z\right)}^{c, 1}$, which is then bounded w.r.t. $z^{\prime}$ and $z$ with values in $\mathcal{M}_{k} S_{\min \left(\rho, \rho^{\prime}, \rho^{\prime \prime}\right)}^{m+m^{\prime}}\left(X \times \mathbf{R}^{n}\right)$. Applying Proposition 2.6 to operator $\mathcal{R}_{\left(z^{\prime}, z\right)}^{c, 1}$, associated with symbol $q_{\left(z^{\prime}, z\right)}^{c, 1}$, which kernel is

$$
R_{\left(z^{\prime}, z\right)}^{c, 1}\left(x^{\prime \prime}, x\right)=\int e^{i\left\langle x^{\prime \prime}-x \mid \xi\right\rangle} q_{\left(z^{\prime}, z\right)}^{c, 1}\left(x^{\prime \prime}, \xi\right) e^{i \Delta b_{1}\left(z, x^{\prime \prime}, \xi\right)} d \xi
$$

we obtain

$$
\left\|\mathcal{R}_{\left(z^{\prime}, z\right)}^{c, 1}\right\|_{\left(\left(H^{(s)}\right)^{k},\left(H^{\left(s-m-m^{\prime}\right)}\right)^{k}\right)} \leq K^{(3)} .
$$

Finally, we have obtained $\mathcal{R}_{\left(z^{\prime}, z\right)}=\mathcal{R}_{\left(z^{\prime}, z\right)}^{b, 1}+\mathcal{R}_{\left(z^{\prime}, z\right)}^{b, 2}+\mathcal{R}_{\left(z^{\prime}, z\right)}^{c, 1}$, which yields the expected estimate,

$$
\left\|\mathcal{R}_{\left(z^{\prime}, z\right)}\right\|_{\left(\left(H^{(s)}\right)^{k},\left(H^{\left(s-m-m^{\prime}\right)}\right)^{k}\right)} \leq K^{(1)}+K^{(2)}+K^{(3)} .
$$

\section{A.6 Proof of Proposition 3.6}

In the proof, we shall always assume that $\Delta$ is sufficiently small to apply the invoked properties and results.

We define

$$
\mathfrak{A}_{\left(z^{\prime}, z\right)}:=\partial_{z^{\prime}} \circ \mathcal{G}_{\left(z^{\prime}, z\right)}, \quad \mathfrak{B}_{\left(z^{\prime}, z\right)}:=a_{z}\left(x, D_{x}\right) \circ \mathcal{G}_{\left(z^{\prime}, z\right)} .
$$

We recall that $\left\|\mathcal{G}_{\left(z^{\prime}, z\right)}\right\|_{\left(\left(H^{(s)}\right)^{k},\left(H^{(s)}\right)^{k}\right)} \leq C$ by Proposition 2.6 uniformly w.r.t. $z^{\prime}$ and $z$. With Assumption 3.5 we then have

$$
\left\|\left(a\left(z, x, D_{x}\right)-a\left(z^{\prime}, x, D_{x}\right)\right) \circ \mathcal{G}_{\left(z^{\prime}, z\right)}\right\|_{\left(\left(H^{(s)}\right)^{k},\left(H^{(s-1)}\right)^{k}\right)} \leq C \Delta^{\alpha} .
$$

It is thus sufficient to prove

$$
\left\|\mathfrak{U}_{\left(z^{\prime}, z\right)}+\mathfrak{B}_{\left(z^{\prime}, z\right)}\right\|_{\left(\left(H^{(s)}\right)^{k},\left(H^{(s-1)}\right)^{k}\right)} \leq C \Delta,
$$

uniformly w.r.t. $z^{\prime}$ and $z$ in $[0, Z]$.

We have

$$
\mathfrak{A}_{\left(z^{\prime}, z\right)}(u)\left(x^{\prime}\right)=-\iint e^{i\left\langle x^{\prime}-x \mid \xi\right\rangle} g_{\left(z^{\prime}, z\right)}\left(x^{\prime}, \xi\right) a\left(z, x^{\prime}, \xi\right) e^{-\Delta a_{1}\left(z, x^{\prime}, \xi\right)} u(x) d \xi d x
$$


With a first-order Taylor formula, we find

$$
g_{\left(z^{\prime}, z\right)}(x, \xi)=I_{k}+\Delta \tilde{g}_{\left(z^{\prime}, z\right)}(x, \xi)
$$

with $\tilde{g}_{\left(z^{\prime}, z\right)}$ bounded w.r.t. $\Delta$ and $z$ with values in $\mathcal{M}_{k} S^{0}\left(\mathbf{R}^{n} \times \mathbf{R}^{n}\right)$. We can thus write $\mathfrak{A}_{\left(z^{\prime}, z\right)}=\underline{\mathfrak{A}}_{\left(z^{\prime}, z\right)}+\Delta \tilde{\mathfrak{A}}\left(z^{\prime}, z\right)$ with

$$
\tilde{\mathfrak{A}}_{\left(z^{\prime}, z\right)}(u)\left(x^{\prime}\right)=-\iint e^{i\left\langle x^{\prime}-x \mid \xi\right\rangle} \tilde{g}_{\left(z^{\prime}, z\right)}\left(x^{\prime}, \xi\right) a\left(z, x^{\prime}, \xi\right) e^{-\Delta a_{1}\left(z, x^{\prime}, \xi\right)} u(x) d \xi d x
$$

and $\mathfrak{\mathfrak { A }}_{\left(z^{\prime}, z\right)}$ is given by the same formula with $\tilde{g}_{\left(z^{\prime}, z\right)}$ replaced by $I_{k}$. We find $\left\|\tilde{\mathfrak{A}}_{\left(z^{\prime}, z\right)}\right\|_{\left(\left(H^{(s)}\right)^{k},\left(H^{(s-1)}\right)^{k}\right)} \leq C$ by Proposition 2.6 since the symbol $\tilde{g}_{\left(z^{\prime}, z\right)}\left(x^{\prime}, \xi\right) a\left(z, x^{\prime}, \xi\right)$ is bounded w.r.t. $z^{\prime}$ and $z$ with values in $\mathcal{M}_{k} S^{1}\left(\mathbf{R}^{n} \times\right.$ $\mathbf{R}^{n}$ ).

By Theorem 3.4, we find

$$
\mathfrak{B}_{\left(z^{\prime}, z\right)}=\mathcal{B}_{\left(z^{\prime}, z\right)}+\Delta \mathcal{R}_{\left(z^{\prime}, z\right)}
$$

with

$$
\left\|\mathcal{R}_{\left(z^{\prime}, z\right)}\right\|_{\left(\left(H^{(s)}\right)^{k},\left(H^{(s-1)}\right)^{k}\right)} \leq C, \quad 0 \leq z \leq z^{\prime} \leq Z
$$

and the operator $\mathcal{B}_{\left(z^{\prime}, z\right)}$ is given by

$$
\mathcal{B}_{\left(z^{\prime}, z\right)} u\left(x^{\prime}\right)=\iint e^{i\left\langle x^{\prime}-x \mid \xi\right\rangle} q_{\left(z^{\prime}, z\right)}\left(x^{\prime}, \xi\right) e^{i \Delta b_{1}\left(z, x^{\prime}, \xi\right)} u(x) d \xi d x
$$

with

(A.18) $q_{\left(z^{\prime}, z\right)}\left(x^{\prime}, \xi\right)=\iint e^{i\left\langle x^{\prime}-y \mid \eta-\xi\right\rangle} a\left(z, x^{\prime}, \eta\right) g_{\left(z^{\prime}, z\right)}(y, \xi) p_{\Delta}(z, y, \xi) w(z, y, \xi) e^{i \Delta\left(d_{b}(z, y, \xi)-d_{b}\left(z, x^{\prime}, \xi\right)\right)} v(z, y, \xi) d \eta d y$,

which is bounded w.r.t. to $z^{\prime}$ and $z$ with values in $\mathcal{M}_{k} S_{\rho}^{1}\left(X \times \mathbf{R}^{n}\right)$.

Making use of (A.16) once more, we obtain

$$
q_{\left(z^{\prime}, z\right)}(x, \xi)=\underline{q}_{\left(z^{\prime}, z\right)}(x, \xi)+\Delta \tilde{q}_{\left(z^{\prime}, z\right)}(x, \xi),
$$

with both $\underline{q}_{\left(z^{\prime}, z\right)}$ and $\tilde{q}_{\left(z^{\prime}, z\right)}$ bounded w.r.t. to $z^{\prime}$ and $z$ with values in $\mathcal{M}_{k} S_{\rho}^{1}\left(X \times \mathbf{R}^{n}\right)$, with $\underline{q}_{\left(z^{\prime}, z\right)}$ and $\tilde{q}_{\left(z^{\prime}, z\right)}$ given by expression (A.18) with the term $g_{\left(z^{\prime}, z\right)}(y, \xi)$ replaced by $I_{k}$ and $\tilde{g}_{\left(z^{\prime}, z\right)}(y, \xi)$, respectively. We then define the operator $\underline{\mathfrak{B}}_{\left(z^{\prime}, z\right)}$ by

$$
\underline{\mathfrak{B}}_{\left(z^{\prime}, z\right)}(u)\left(x^{\prime}\right):=\iint e^{i\left\langle x^{\prime}-x \mid \xi\right\rangle} \underline{q}_{\left(z^{\prime}, z\right)}\left(x^{\prime}, \xi\right) e^{i \Delta b_{1}\left(z, x^{\prime}, \xi\right)} u(x) d \xi d x,
$$

and the operator $\tilde{\mathfrak{B}}_{\left(z^{\prime}, z\right)}$ by the same formula with $\underline{q}_{\left(z^{\prime}, z\right)}$ replaced by $\tilde{q}_{\left(z^{\prime}, z\right)}$. We have $\mathcal{B}_{\left(z^{\prime}, z\right)}=\underline{B}_{\left(z^{\prime}, z\right)}+\Delta \tilde{\mathfrak{B}}_{\left(z^{\prime}, z\right)}$ and $\left\|\tilde{\mathfrak{B}}_{\left(z^{\prime}, z\right)}\right\|_{\left(\left(H^{(s)}\right)^{k},\left(H^{(s-1)}\right)^{k}\right)} \leq C$ for some $C \geq 0$.

It is now sufficient to prove

$$
\left\|\underline{\mathscr{A}}_{\left(z^{\prime}, z\right)}+\underline{\mathfrak{B}}_{\left(z^{\prime}, z\right)}\right\|_{\left(\left(H^{(s)}\right)^{k},\left(H^{(s-1)}\right)^{k}\right)} \leq C \Delta, \quad 0 \leq z \leq z^{\prime} \leq Z,
$$

when $\Delta=z^{\prime}-z$ is sufficiently small. We may therefore conclude by the second part of Proposition 2.6 and the following lemma.

Lemma A.3. The matrix symbol

$$
\kappa_{\Delta}(z, x, \xi):=\underline{q}_{\left(z^{\prime}, z\right)}(x, \xi)-a(z, x, \xi) p_{\Delta}(z, x, \xi)
$$

is equal to $\Delta \tilde{\kappa}_{\Delta}(z, x, \xi)$ with $\tilde{\kappa}_{\Delta}(z, x, \xi)$ bounded w.r.t. $\Delta$ and $z$ with values in $\mathcal{M}_{k} S_{\rho}^{1}\left(\mathbf{R}^{n} \times \mathbf{R}^{n}\right)$. 
Proof. We first write $\kappa_{\Delta}(z, x, \xi)=\kappa_{\Delta, 1}(z, x, \xi)+\kappa_{\Delta, 2}(z, x, \xi)$ with

$$
\begin{aligned}
& \kappa_{\Delta, 1}(z, x, \xi):=\underline{q}_{\left(z^{\prime}, z\right)}(x, \xi)-\left(a(z, .) \# p_{\Delta}(z, .)\right)(x, \xi), \\
& \kappa_{\Delta, 2}(z, x, \xi):=\left(a(z, .) \# p_{\Delta}(z, .)\right)(x, \xi)-a(z, x, \xi) p_{\Delta}(z, x, \xi),
\end{aligned}
$$

and work on each term separately.

The composition product of $\psi$ DOs [15, Theorem 18.1.8] gives the following oscillatory integral representation

$$
\left(a(z, .) \# p_{\Delta}(z, .)\right)(x, \xi)=\iint e^{i\langle x-y \mid \eta-\xi\rangle} a(z, x, \eta) p_{\Delta}(z, y, \xi) d \eta d y .
$$

We thus obtain

$$
\kappa_{\Delta, 1}(z, x, \xi)=\iint e^{i\langle x-y \mid \eta-\xi\rangle} a(z, x, \eta) p_{\Delta}(z, y, \xi) w(z, y, \xi)\left(e^{i \Delta\left(d_{b}(z, y, \xi)-d_{b}(z, x, \xi)\right)}-I_{k}\right) v(z, y, \xi) d \eta d y,
$$

which we write

$$
\kappa_{\Delta, 1}(z, x, \xi)=i \Delta \int_{0}^{1} \iint e^{i\langle x-y \mid \eta-\xi\rangle} a(z, x, \eta) p_{\Delta}(z, y, \xi) w(z, y, \xi) \mu(z, x, y, \xi) e^{i t \Delta \mu(z, x, y, \xi)} v(z, y, \xi) d \eta d y d t,
$$

where

$$
\mu(z, x, y, \xi):=d_{b}(z, y, \xi)-d_{b}(z, x, \xi)=\langle y-x \mid h(z, x, y, \xi)\rangle
$$

for $h(z, x, y, \xi)$ continuous w.r.t. $z$ with values in $\left(\mathcal{M}_{k} S^{1}\left(X \times X \times \mathbf{R}^{n}\right)\right)^{n}$, homogeneous of degree 1 by estimate $(1.1 .9)$ in [16]. We observe that

$$
\kappa_{\Delta, 1}(z, x, \xi)=-\Delta \iint_{0}^{1} \iint_{\eta} e^{i\langle x-y \mid \eta-\xi\rangle}\left|a(z, x, \eta) p_{\Delta}(z, y, \xi) w(z, y, \xi) h(z, x, y, \xi)\right\rangle e^{i t \Delta \mu(z, x, y, \xi)} v(z, y, \xi) d \eta d y d t
$$

which after integration by parts gives

$$
\begin{array}{r}
\kappa_{\Delta, 1}(z, x, \xi)=\Delta \iiint_{0}^{1}\left\langle e^{i\langle x-y \mid \eta-\xi\rangle}\left\langle\nabla_{\eta} a(z, x, \eta) \mid p_{\Delta}(z, y, \xi) w(z, y, \xi) h\left(z, x^{\prime}, y, \xi\right)\right\rangle\right. \\
\times\left. e^{i t \Delta \mu(z, x, y, \xi)} v(z, y, \xi) d \eta d y d t\right|_{x^{\prime}=x},
\end{array}
$$

Because of the diagonal form of the term $e^{i s \Delta \mu(z, x, y, \xi)}$, the result of Proposition 1.10 for the scalar case, can be applied. The first composition formula in Proposition 1.10 with $x^{\prime}, t$ and $z$ as parameters (see also Remark 1.11) yields $\kappa_{\Delta, 1}(z, x, \xi)=\Delta \tilde{\kappa}_{\Delta, 1}(z, x, \xi)$ with $\tilde{\kappa}_{\Delta, 1}(z, x, \xi)$ continuous w.r.t. $z$ and bounded w.r.t. $\Delta$ with values in $\mathcal{M}_{k} S_{\rho}^{1}\left(\mathbf{R}^{n} \times \mathbf{R}^{n}\right)$.

For the second term $\kappa_{\Delta, 2}(z, x, \xi)$, since $p_{\Delta}(z, x, \xi)$ given in (3.1) satisfies Property $\left(Q_{L}\right)$, we can apply Lemma 2.19 with $m=\rho$ and obtain $\kappa_{\Delta, 2}(z, x, \xi)=\Delta \tilde{\kappa}_{\Delta, 2}(z, x, \xi)$ with $\tilde{\kappa}_{\Delta, 2}(z, x, \xi)$ bounded w.r.t. $z$ and $\Delta$ with values in $S_{\rho}^{1}\left(\mathbf{R}^{n} \times \mathbf{R}^{n}\right)$.

\section{A.7 Proofs of Propositions 4.4 and 4.5}

Proof of Proposition 4.4. In the proof, we shall denote by $q$ a seminorm on $S_{\rho^{\prime}}^{m, m^{\prime}}\left(X^{\prime} \times \mathbf{R}^{n} \times X \times \mathbf{R}^{n}\right)$ and by $p$ a seminorm on $\mathscr{P}\left(\mathbf{R}^{n}\right)$, which may change from one line to another.

We set

$v\left(x^{\prime}, x, \xi\right):=\iint e^{i\langle x-y \mid \eta\rangle} e^{-\Delta \underline{h}_{1}(z, x, \eta)} \sigma_{\left(z^{\prime \prime}, z^{\prime}, z\right)}\left(x^{\prime}, \xi, x, \eta\right) u(y) d y d \eta=\int e^{i\langle x \mid \eta\rangle} e^{-\Delta \underline{h}_{1}(z, x, \eta)} \sigma_{\left(z^{\prime \prime}, z^{\prime}, z\right)}\left(x^{\prime}, \xi, x, \eta\right) \hat{u}(\eta) d \eta$, 
which is well defined since $\hat{u} \in \mathscr{P}\left(\mathbf{R}^{n}\right)$. By Proposition 1.6, for all $\alpha, \alpha^{\prime}, \beta$ multi-indices and all $N \geq 0$, there exist $p$ and $q$ such that

$$
\left|\langle x\rangle^{N} \partial_{x^{\prime}}^{\alpha^{\prime}} \partial_{x}^{\alpha} \partial_{\xi}^{\beta} v\left(x^{\prime}, x, \xi\right)\right| \leq C q\left(\sigma_{\left(z^{\prime \prime}, z^{\prime}, z\right)}\right) p(u)\langle\xi\rangle^{m-\rho^{\prime}|\beta|+\delta^{\prime}\left|\alpha^{\prime}\right|}, \quad x^{\prime}, x \in \mathbf{R}^{n}, \xi \in \mathbf{R}^{n},
$$

with $\delta^{\prime}=1-\rho^{\prime}$ (Note that we have used (4.1) here).

Defining $\hat{v}$ as the Fourier transform of $v$ w.r.t. $x$ we obtain for all multi-indices $\alpha, \alpha^{\prime}, \beta$ and all $N \geq 0$ we have

$$
\left|\langle\eta\rangle^{N} \partial_{x^{\prime}}^{\alpha^{\prime}} \partial_{\eta}^{\alpha} \partial_{\xi}^{\beta} \hat{v}\left(x^{\prime}, \eta, \xi\right)\right| \leq C q\left(\sigma_{\left(z^{\prime \prime}, z^{\prime}, z\right)}\right) p(u)\langle\xi\rangle^{m-\rho^{\prime}|\beta|+\delta^{\prime}\left|\alpha^{\prime}\right|}, \quad x^{\prime} \in \mathbf{R}^{n}, \eta, \xi \in \mathbf{R}^{n},
$$

and thus for all multi-indices $\alpha^{\prime}, \beta$ and all $N \geq 0$ we have

$$
\left|\langle\xi\rangle^{N} \partial_{x^{\prime}}^{\alpha^{\prime}} \partial_{\xi}^{\beta} \hat{v}\left(x^{\prime}, \xi, \xi\right)\right| \leq C q\left(\sigma_{\left(z^{\prime \prime}, z^{\prime}, z\right)}\right) p(u), \quad x^{\prime} \in \mathbf{R}^{n}, \xi \in \mathbf{R}^{n}
$$

We now define

$$
w\left(x, x^{\prime}\right):=\int e^{i\langle x \mid \xi\rangle} e^{-\Delta^{\prime} h_{1}\left(z^{\prime}, x, \xi\right)} \hat{v}\left(x^{\prime}, \xi, \xi\right) d \xi
$$

We have $\mathcal{F}_{\left(z^{\prime \prime}, z^{\prime}, z\right)}(u)\left(x^{\prime}\right)=w\left(x^{\prime}, x^{\prime}\right)$. Applying Proposition 1.6 once more, we find for all multi-indices $\alpha^{\prime}, \alpha$ and all $N \geq 0$

$$
\left|\langle x\rangle^{N} \partial_{x}^{\alpha} \partial_{x^{\prime}}^{\alpha^{\prime}} w\left(x, x^{\prime}\right)\right| \leq C q\left(\sigma_{\left(z^{\prime \prime}, z^{\prime}, z\right)}\right) p(u), \quad x, x^{\prime} \in \mathbf{R}^{n},
$$

which concludes the proof.

Proof of Proposition 4.5. The kernel of $\mathcal{H}_{\left(z^{\prime \prime}, z^{\prime}, z\right)}:=\mathcal{F}_{\left(z^{\prime \prime}, z^{\prime}, z\right)} \circ E^{(-s)}$ is given by

$$
H_{\left(z^{\prime \prime}, z^{\prime}, z\right)}\left(x^{\prime}, y\right)=\int_{3} e^{i\left\langle x^{\prime}-x \mid \xi\right\rangle} e^{i \Delta^{\prime} k_{1}\left(z^{\prime}, x^{\prime}, \xi\right)} e^{i\langle x-y \mid \eta\rangle} e^{i \Delta k_{1}(z, x, \eta)} \underline{\sigma}_{\left(z^{\prime \prime}, z^{\prime}, z\right)}\left(x^{\prime}, \xi, x, \eta\right) d \eta d x d \xi,
$$

with

$$
\underline{\sigma}_{\left(z^{\prime \prime}, z^{\prime}, z\right)}\left(x^{\prime}, \xi, x, \eta\right)=\sigma_{\left(z^{\prime \prime}, z^{\prime}, z\right)}\left(x^{\prime}, \xi, x, \eta\right) e^{-\Delta^{\prime} l_{1}\left(z^{\prime}, x^{\prime}, \xi\right)} e^{-\Delta l_{1}(z, x, \eta)}\langle\eta\rangle^{-s},
$$

which is bounded w.r.t. to $z^{\prime \prime}, z^{\prime}$ and $z$ with values in $S_{\min \left(\rho, \rho^{\prime}\right)}^{m, m^{\prime}-s}\left(X^{\prime} \times \mathbf{R}^{n} \times X \times \mathbf{R}^{n}\right)$. The kernel of the adjoint operator $\mathcal{H}_{\left(z^{\prime \prime}, z^{\prime}, z\right)}^{*}$ is given by

$$
H_{\left(z^{\prime \prime}, z^{\prime}, z\right)}^{*}\left(y, t^{\prime}\right)=\int_{\text {(3) }} e^{i\left\langle y-t \mid \eta^{\prime}\right\rangle} e^{-i \Delta \underline{k}_{1}\left(z, t, \eta^{\prime}\right)} e^{i\left\langle t-t^{\prime} \mid \xi^{\prime}\right\rangle} e^{-i \Delta^{\prime} k_{1}\left(z^{\prime}, t^{\prime}, \xi^{\prime}\right)} \bar{\sigma}_{\left(z^{\prime \prime}, z^{\prime}, z\right)}\left(t^{\prime}, \xi^{\prime}, t, \eta^{\prime}\right) d \xi^{\prime} d t d \eta^{\prime}
$$

The kernel of $\mathcal{H}_{\left(z^{\prime \prime}, z^{\prime}, z\right)} \circ \mathcal{H}_{\left(z^{\prime \prime}, z^{\prime}, z\right)}^{*}$ is given by

$$
\begin{aligned}
K_{\left(z^{\prime \prime}, z^{\prime}, z\right)}\left(x^{\prime}, t^{\prime}\right)= & \int_{5} e^{i\left\langle x^{\prime}-x \mid \xi\right\rangle} e^{i \Delta^{\prime} k_{1}\left(z^{\prime}, x^{\prime}, \xi\right)} e^{i\langle x-t \mid \eta\rangle} e^{i \Delta\left(\underline{k}_{1}(z, x, \eta)-\underline{k}_{1}(z, t, \eta)\right)} \\
& \times \underline{\sigma}_{\left(z^{\prime \prime}, z^{\prime}, z\right)}\left(x^{\prime}, \xi, x, \eta\right) \underline{\sigma}_{\left(z^{\prime \prime}, z^{\prime}, z\right)}\left(t^{\prime}, \xi^{\prime}, t, \eta\right) e^{i\left\langle t-t^{\prime} \mid \xi^{\prime}\right\rangle} e^{-i \Delta^{\prime} k_{1}\left(z^{\prime}, t^{\prime}, \xi^{\prime}\right)} d \eta d x d \xi d \xi^{\prime} d t .
\end{aligned}
$$

(We freely interchange the order of integration, as is usually done with oscillatory integrals without introducing regularization cutoff functions explicitly [16, Section 7.8].)

As in the proof of Theorem 2.22, with Taylor's formula, we write

$$
\underline{k}_{1}(z, x, \eta)-\underline{k}_{1}(z, t, \eta)=\langle x-t \mid \tilde{k}(z, x, t, \eta)\rangle
$$

and perform the global change of variables $\eta \rightarrow \eta+\Delta \tilde{k}(z, x, t, \eta)=H_{(z, \Delta, x, t)}(\eta)$. We denote

$$
\tilde{\eta}(\Delta, z, x, t, \eta)=H_{(z, \Delta, x, t)}^{-1}(\eta),
$$


which we write $\tilde{\eta}$ for the sake of concision. We have

$$
\begin{aligned}
K_{\left(z^{\prime \prime}, z^{\prime}, z\right)}\left(x^{\prime}, t^{\prime}\right)=\int_{\odot} e^{i\left\langle x^{\prime}-x \mid \xi\right\rangle} e^{i \Delta^{\prime} k_{1}\left(z^{\prime}, x^{\prime}, \xi\right)} e^{i\langle x-t \mid \eta\rangle} \underline{\sigma}_{\left(z^{\prime \prime}, z^{\prime}, z\right)}\left(x^{\prime}, \xi, x, \tilde{\eta}\right) \\
\quad \times \underline{\sigma}_{\left(z^{\prime \prime}, z^{\prime}, z\right)}\left(t^{\prime}, \xi^{\prime}, t, \tilde{\eta}\right) e^{i\left\langle t-t^{\prime} \mid \xi^{\prime}\right\rangle} e^{-i \Delta^{\prime} k_{1}\left(z^{\prime}, t^{\prime}, \xi^{\prime}\right)} J_{\Delta}(z, x, t, \eta) d \eta d x d \xi d \xi^{\prime} d t,
\end{aligned}
$$

where $J_{\Delta}(z, x, t, \eta)$ is the Jacobian which is bounded w.r.t. $z^{\prime}$ and $z$ with values in $S^{0}\left(X \times T \times \mathbf{R}^{n}\right)$. By Lemma 1.7 (adapting the proof to multiple symbols), $\underline{\sigma}_{\left(z^{\prime \prime}, z^{\prime}, z\right)}\left(x^{\prime}, \xi, x, \tilde{\eta}\right)=: f_{1}\left(x^{\prime}, \xi, x, t, \eta\right)$ and $\underline{\sigma}_{\left(z^{\prime \prime}, z^{\prime}, z\right)}\left(t^{\prime}, \xi^{\prime}, t, \tilde{\eta}\right)=$ : $f_{2}\left(t^{\prime}, \xi^{\prime}, x, t, \eta\right)$ are bounded w.r.t. $z^{\prime \prime}, z^{\prime}$ and $z$ with values in $S_{\min \left(\rho, \rho^{\prime}\right)}^{m, m^{\prime}-s}\left(X^{\prime} \times \mathbf{R}^{n} \times(X \times T) \times \mathbf{R}^{n}\right)$ and $S_{\min \left(\rho, \rho^{\prime}\right)}^{m, m^{\prime}-s}\left(T^{\prime} \times\right.$ $\left.\mathbf{R}^{n} \times(X \times T) \times \mathbf{R}^{n}\right)$ respectively.

We then observe that

$$
K_{\left(z^{\prime \prime}, z^{\prime}, z\right)}\left(x^{\prime}, t^{\prime}\right)=\int_{3} e^{i\left\langle x^{\prime}-x \mid \xi\right\rangle} e^{i \Delta^{\prime} k_{1}\left(z^{\prime}, x^{\prime}, \xi\right)} \rho_{\left(z^{\prime \prime}, z^{\prime}, z\right)}\left(x^{\prime}, \xi, x, t^{\prime}, \xi^{\prime}\right) e^{i\left\langle x-t^{\prime} \mid \xi^{\prime}\right\rangle} e^{-i \Delta^{\prime} k_{1}\left(z^{\prime}, t^{\prime}, \xi^{\prime}\right)} d x d \xi d \xi^{\prime}
$$

with

$$
\rho_{\left(z^{\prime \prime}, z^{\prime}, z\right)}\left(x^{\prime}, \xi, x, t^{\prime}, \xi^{\prime}\right)=\iint e^{i\left\langle x-t \mid \eta-\xi^{\prime}\right\rangle} \underline{\sigma}_{\left(z^{\prime \prime}, z^{\prime}, z\right)}\left(x^{\prime}, \xi, x, \tilde{\eta}\right) \underline{\sigma}_{\left(z^{\prime \prime}, z^{\prime}, z\right)}\left(t^{\prime}, \xi^{\prime}, t, \tilde{\eta}\right) J_{\Delta}(z, x, t, \eta) d t d \eta .
$$

Adapting the proof of Theorem 18.1.8 in [15], we find that $\rho_{\left(z^{\prime \prime}, z^{\prime}, z\right)}\left(x^{\prime}, \xi, x, t^{\prime}, \xi^{\prime}\right)$ is a multiple symbol which is bounded w.r.t. $z^{\prime \prime}, z^{\prime}$ and $z$ with values in $S_{\min \left(\rho^{\prime}, \rho\right)}^{m, m+2\left(m^{\prime}-s\right)}\left(X^{\prime} \times \mathbf{R}^{n} \times\left(X \times T^{\prime}\right) \times \mathbf{R}^{n}\right)$.

Now, adapting the proof of Lemma 1.13 to the case of a multiple symbol we now find that the operator $\mathcal{K}_{\left(z^{\prime \prime}, z^{\prime}, z\right)}$ with kernel $K_{\left(z^{\prime \prime}, z^{\prime}, z\right)}\left(x^{\prime}, t^{\prime}\right)$ is a pseudodifferential operator with an amplitude bounded w.r.t. $z^{\prime \prime}, z^{\prime}$ and $z$ with values in $S_{\min \left(\rho, \rho^{\prime}\right)}^{2\left(m+m^{\prime}-s\right)}\left(X^{\prime} \times T^{\prime} \times \mathbf{R}^{n}\right)$.

Composing $\mathcal{K}_{\left(z^{\prime \prime} z^{\prime}, z\right)}$ on both sides by $E^{\left(s-m-m^{\prime}\right)}$ we find that $E^{\left(s-m-m^{\prime}\right)} \circ \mathcal{F}_{\left(z^{\prime \prime}, z^{\prime}, z\right)} \circ E^{(s)}$ is bounded from $L^{2}$ into $L^{2}$ by the Calderón-Vaillancourt Theorem (see [23, Chapter 7, Sections 1,2] or [39, Section XIII-2]).

\section{A.8 Proofs of Propositions 4.6, 4.7 and 4.8}

Proof of Proposition 4.6. In the proof, we shall always implicitly assume that $\Delta$ and $\Delta^{\prime}$ are taken sufficiently small to apply the invoked properties. As in the proof of Theorem 2.22, we observe that there is no loss of generality in assuming $g_{\left(z^{\prime \prime}, z^{\prime}\right)}(x, \xi)=g_{\left(z^{\prime}, z\right)}(x, \xi)=I_{k}$.

With Assumption 4.2, we write $L\left(z, x^{\prime}, \xi\right)=L\left(z^{\prime}, x^{\prime}, \xi\right)+\left(z-z^{\prime}\right) \tilde{L}\left(z^{\prime}, z, x^{\prime}, \xi\right)$ which yields

$$
\mathcal{H}_{\left(z^{\prime}, z\right)}^{l r}(u)\left(x^{\prime}\right)=\iint e^{i\left\langle x^{\prime}-x \mid \xi\right\rangle} L\left(z^{\prime}, x^{\prime}, \xi\right) e^{-\Delta \alpha_{1}\left(z, x^{\prime}, \xi\right)} R\left(z, x^{\prime}, \xi\right) u(x) d x d \xi+\Delta \mathcal{K}_{\left(z^{\prime}, z\right)}^{a}(u)\left(x^{\prime}\right),
$$

with $\left\|\mathcal{K}_{\left(z^{\prime}, z\right)}^{a}\right\|_{\left(\left(H^{(s)}\right)^{k},\left(H^{\left.(s))^{k}\right)}\right.\right.} \leq C$, for some $C \geq 0$, by Theorem 1.12, since $\alpha_{1}$ satisfies Assumption 2.2.

Since $\left\|\mathcal{H}_{\left(z^{\prime \prime}, z^{\prime}\right)}^{l r}\right\|_{\left(\left(H^{(s)}\right)^{k},\left(H^{(s)}\right)^{k}\right)} \leq C$, it thus suffices to consider the composition of $\mathcal{H}_{\left(z^{\prime \prime}, z^{\prime}\right)}^{l r}$ with the first term in (A.20). We call $\mathcal{F}_{\left(z^{\prime \prime}, z^{\prime}, z\right)}$ the resulting operator. It is formally given by

$$
\mathcal{F}_{\left(z^{\prime \prime}, z^{\prime}, z\right)}(u)\left(x^{\prime}\right)=\iint e^{i\left\langle x^{\prime}-x \mid \eta\right\rangle} f_{\left(z^{\prime \prime}, z^{\prime}, z\right)}\left(x^{\prime}, \eta\right) u(x) d x d \eta,
$$

with

$$
f_{\left(z^{\prime \prime}, z^{\prime}, z\right)}\left(x^{\prime}, \eta\right)=\iint e^{i\left\langle x^{\prime}-y \mid \xi-\eta\right\rangle} L\left(z^{\prime}, x^{\prime}, \xi\right) e^{-\Delta^{\prime} \alpha_{1}\left(z^{\prime}, x^{\prime}, \xi\right)} R\left(z^{\prime}, x^{\prime}, \xi\right) L\left(z^{\prime}, y, \eta\right) e^{-\Delta \alpha_{1}(z, y, \eta)} R(z, y, \eta) d y d \xi .
$$

From the regularity of $L(z, x, \xi)$ w.r.t. the variables $x$ and $\xi$ that we assumed, we write

$$
L\left(z^{\prime}, y, \eta\right)=L\left(z^{\prime}, x^{\prime}, \xi\right)+\left\langle y-x^{\prime} \mid L_{1}\left(z^{\prime}, x^{\prime}, \xi, y, \eta\right)\right\rangle+\left\langle\eta-\xi \mid L_{2}\left(z^{\prime}, x^{\prime}, \xi, y, \eta\right)\right\rangle
$$

with Taylor's formula. We then have $f_{\left(z^{\prime \prime}, z^{\prime}, z\right)}\left(x^{\prime}, \eta\right)=f_{\left(z^{\prime \prime}, z^{\prime}, z\right), a}\left(x^{\prime}, \eta\right)+f_{\left(z^{\prime \prime}, z^{\prime}, z\right), b}\left(x^{\prime}, \eta\right)$ where

$$
f_{\left(z^{\prime \prime}, z^{\prime}, z\right), a}\left(x^{\prime}, \eta\right)=\iint e^{i\left\langle x^{\prime}-y \mid \xi-\eta\right\rangle} L\left(z^{\prime}, x^{\prime}, \xi\right) e^{-\Delta^{\prime} \alpha_{1}\left(z^{\prime}, x^{\prime}, \xi\right)} e^{-\Delta \alpha_{1}(z, y, \eta)} R(z, y, \eta) d y d \xi,
$$


and

$$
\begin{aligned}
f_{\left(z^{\prime \prime}, z^{\prime}, z\right), b}\left(x^{\prime}, \eta\right)=\iint e^{i\left\langle x^{\prime}-y \mid \xi-\eta\right\rangle} L\left(z^{\prime}, x^{\prime}, \xi\right) e^{-\Delta^{\prime} \alpha_{1}\left(z^{\prime}, x^{\prime}, \xi\right)} R\left(z^{\prime}, x^{\prime}, \xi\right) & \\
& \quad \times\left(\left\langle y-x^{\prime} \mid L_{1}\left(z^{\prime}, x^{\prime}, \xi, y, \eta\right)\right\rangle+\left\langle\eta-\xi \mid L_{2}\left(z^{\prime}, x^{\prime}, \xi, y, \eta\right)\right\rangle\right) e^{-\Delta \alpha_{1}(z, y, \eta)} R(z, y, \eta) d y d \xi .
\end{aligned}
$$

The part of the operator associated to $f_{\left(z^{\prime \prime}, z^{\prime}, z\right), a}\left(x^{\prime}, \eta\right)$ corresponds to $\mathcal{H}_{\left(z^{\prime \prime}, z^{\prime}\right)}^{l} \circ \mathcal{H}_{\left(z^{\prime}, z\right)}^{r}$. We now study the second part associated to $f_{\left(z^{\prime \prime}, z^{\prime}, z\right), b}\left(x^{\prime}, \eta\right)$. As in the proof of Lemma 1.13, we introduce $\chi \in \mathscr{C}_{c}^{\infty}\left(\mathbf{R}^{n}\right)$ be such that $\chi(\xi)=1$ if $|\xi| \leq \frac{1}{2}$ and $\chi(\xi)=0$ if $|\xi| \geq \frac{2}{3}$ and set $\chi_{\infty}(\eta, \xi):=1-\chi\left(\frac{\eta-\xi}{(1-\tau)(\xi)}\right)$, with $0<\tau<1$. We have $f_{\left(z^{\prime \prime}, z^{\prime}, z\right), b}\left(x^{\prime}, \eta\right)=f_{\left(z^{\prime \prime}, z^{\prime}, z\right), b, \infty}\left(x^{\prime}, \eta\right)+f_{\left(z^{\prime \prime}, z^{\prime}, z\right), b, 0}\left(x^{\prime}, \eta\right)$ where

$$
\begin{aligned}
f_{\left(z^{\prime \prime}, z^{\prime}, z\right), b, \infty}\left(x^{\prime}, \eta\right)=\iint & e^{i\left\langle x^{\prime}-y \mid \xi-\eta\right\rangle} \chi_{\infty}(\eta, \xi) L\left(z^{\prime}, x^{\prime}, \xi\right) e^{-\Delta^{\prime} \alpha_{1}\left(z^{\prime}, x^{\prime}, \xi\right)} R\left(z^{\prime}, x^{\prime}, \xi\right) \\
& \times\left(\left\langle y-x^{\prime} \mid L_{1}\left(z^{\prime}, x^{\prime}, \xi, y, \eta\right)\right\rangle+\left\langle\eta-\xi \mid L_{2}\left(z^{\prime}, x^{\prime}, \xi, y, \eta\right)\right\rangle\right) e^{-\Delta \alpha_{1}(z, y, \eta)} R(z, y, \eta) d y d \xi
\end{aligned}
$$

with a similar definition for $f_{\left(z^{\prime \prime}, z^{\prime}, z\right), b, 0}\left(x^{\prime}, \eta\right)$ with $\chi_{\infty}(\eta, \xi)$ replaced by $\chi_{0}(\eta, \xi)=\chi\left(\frac{\eta-\xi}{(1-\tau)\langle\xi\rangle}\right)=1-\chi_{\infty}(\eta, \xi)$. We consider the component $\left(f_{\left(z^{\prime \prime}, z^{\prime}, z\right), b, \infty}\right)_{i j}\left(x^{\prime}, \eta\right)$, for $1 \leq i, j \leq k$. It is a sum of terms of the form

$$
\iint e^{i \varphi\left(x^{\prime}, y, \xi, \eta\right)} \chi_{\infty}(\eta, \xi) \sigma_{1}\left(z^{\prime}, x^{\prime}, \xi\right) \sigma_{2}\left(z^{\prime}, x^{\prime}, \xi, y, \eta\right) \sigma_{3}(z, y, \eta) d y d \xi
$$

with $\varphi\left(x^{\prime}, y, \xi, \eta\right)=\left\langle x^{\prime}-y \mid \xi-\eta\right\rangle+\Delta^{\prime} d_{\beta, l}\left(z^{\prime}, x^{\prime}, \xi\right)+\Delta d_{\beta, m}(z, y, \eta)$, for $1 \leq l, m \leq k$ (we have used Assumptions 4.1 and 2.2 made on $\alpha_{1}$ and the notations that were introduced in Section 2). Since $e^{-\Delta^{\prime} d_{\gamma, l}\left(z^{\prime}, x^{\prime}, \xi\right)}$ and $e^{-\Delta^{\prime} d_{\gamma, m}(z, y, \eta)}$ are bounded w.r.t. $z^{\prime}$ and $z$ with values in $S_{\rho}^{0}$ we may take $\sigma_{1}\left(z^{\prime}, x^{\prime}, \xi\right)$ and $\sigma_{3}(z, y, \eta)$ to be bounded w.r.t. $z^{\prime}$ and $z$ with values in $S_{\rho}^{0}\left(X^{\prime} \times \mathbf{R}^{n}\right)$ and $S_{\rho}^{0}\left(Y \times \mathbf{R}^{n}\right)$ respectively and the term $\sigma_{2}\left(z^{\prime}, x^{\prime}, \xi, y, \eta\right)$ to be bounded w.r.t. $z^{\prime}$ with values in the space of multiple symbols $S^{0,0}\left(X^{\prime} \times \mathbf{R}^{n} \times Y \times \mathbf{R}^{n}\right)$.

We observe that $\left|\nabla_{x^{\prime}} \varphi\right| \leq C\langle\xi-\eta ; \xi\rangle$ and $\left|\nabla_{\eta} \varphi\right| \leq C\left\langle x^{\prime}-y\right\rangle$. We thus have

$$
\left|\partial_{x^{\prime}}^{\beta} \partial_{\eta}^{\alpha} e^{i \varphi\left(x^{\prime}, y, \xi, \eta\right)}\right| \leq C\langle\xi-\eta ; \xi\rangle^{|\beta|}\left\langle x^{\prime}-y\right\rangle^{|\alpha|} .
$$

We note that

$$
\left|\nabla_{\xi} \varphi\right| \leq C\left\langle x^{\prime}-y\right\rangle
$$

and we see that

$$
\begin{aligned}
\nabla_{y} \varphi & =-(\xi-\eta)+\Delta \nabla_{y} d_{\beta, m}(z, y, \eta) \\
& =-(\xi-\eta)+\Delta\left(\nabla_{y} d_{\beta, m}(z, y, \eta)-\nabla_{y} d_{\beta, m}(z, y, \xi)\right)+\Delta \nabla_{y} d_{\beta, m}(z, y, \xi) .
\end{aligned}
$$

With Taylor's formula we have, for $\Delta$ sufficiently small,

$$
\left|-(\xi-\eta)+\Delta\left(\nabla_{y} d_{\beta, m}(z, y, \eta)-\nabla_{y} d_{\beta, m}(z, y, \xi)\right)\right| \geq C|\eta-\xi| .
$$

We also have

$$
\left|\Delta \nabla_{y} d_{\beta, m}(z, y, \xi)\right| \leq \Delta\langle\xi\rangle
$$

Thus, for $\Delta$ sufficiently small, on the support of $\chi_{\infty}(\eta, \xi)$, where $|\eta-\xi| \geq \frac{1}{2}(1-\tau)\langle\xi\rangle$, the previous estimates yield

$$
\left|\nabla_{y} \varphi\right| \geq C|\eta-\xi| \geq C^{\prime}\langle\xi\rangle .
$$

Estimates (A.21), (A.22) and (A.23) allow to conclude that $f_{\left(z^{\prime \prime}, z^{\prime}, z\right), b, \infty}\left(x^{\prime}, \eta\right)$ is bounded w.r.t. $z^{\prime \prime}, z^{\prime}$ and $z$ with values in $S^{-\infty}\left(X^{\prime} \times \mathbf{R}^{n}\right)$, as in the proof of Lemma 1.13. First-order Taylor expansions of the terms $e^{-\Delta^{\prime} \alpha_{1}\left(z^{\prime}, x^{\prime}, \xi\right)}$ and $e^{-\Delta \alpha_{1}(z, y, \eta)}$, w.r.t. to $\Delta^{\prime}$ and $\Delta$ respectively, in the expression of $f_{\left(z^{\prime \prime}, z^{\prime}, z\right), b, \infty}\left(x^{\prime}, \eta\right)$ thus give

$$
f_{\left(z^{\prime \prime}, z^{\prime}, z\right), b, \infty}\left(x^{\prime}, \eta\right)=f_{\left(z^{\prime \prime}, z^{\prime}, z\right), c, \infty}\left(x^{\prime}, \eta\right)+\max \left(\Delta, \Delta^{\prime}\right) f_{\left(z^{\prime \prime}, z^{\prime}, z\right), 3, \infty}\left(x^{\prime}, \eta\right),
$$


where

$$
\begin{aligned}
f_{\left(z^{\prime \prime}, z^{\prime}, z\right), c, \infty}\left(x^{\prime}, \eta\right) & =\iint e^{i\left\langle x^{\prime}-y \mid \xi-\eta\right\rangle} \chi_{\infty}(\eta, \xi) L\left(z^{\prime}, x^{\prime}, \xi\right) R\left(z^{\prime}, x^{\prime}, \xi\right) \\
& \times\left(\left\langle y-x^{\prime} \mid L_{1}\left(z^{\prime}, x^{\prime}, \xi, y, \eta\right)\right\rangle+\left\langle\eta-\xi \mid L_{2}\left(z^{\prime}, x^{\prime}, \xi, y, \eta\right)\right\rangle\right) R(z, y, \eta) d y d \xi \\
& =\iint e^{i\left\langle x^{\prime}-y \mid \xi-\eta\right\rangle} \chi_{\infty}(\eta, \xi)\left(L\left(z^{\prime}, y, \eta\right)-L\left(z^{\prime}, x^{\prime}, \xi\right)\right) R(z, y, \eta) d y d \xi
\end{aligned}
$$

and $f_{\left(z^{\prime \prime}, z^{\prime}, z\right), 3, \infty}\left(x^{\prime}, \eta\right)$ is bounded w.r.t. $z^{\prime \prime}, z^{\prime}$ and $z$ with values in $S^{-\infty}\left(X^{\prime} \times \mathbf{R}^{n}\right)$.

We now consider the term $f_{\left(z^{\prime \prime}, z^{\prime}, z\right), b, 0}\left(x^{\prime}, \eta\right)$. In the support of $\chi_{0}(\eta, \xi)$, for $1-\tau$ small, we have

$$
C\langle\xi\rangle \leq\langle\eta\rangle \leq C^{\prime}\langle\xi\rangle .
$$

We then note that $L\left(z^{\prime}, x^{\prime}, \xi\right), R\left(z^{\prime}, x^{\prime}, \xi\right), L_{1}\left(z^{\prime}, x^{\prime}, \xi, y, \eta\right), R(z, y, \eta)$ satisfy the estimates of symbols of order 0 w.r.t. to the variables $x^{\prime}, y$ and $\xi$ (or equivalently w.r.t. $x^{\prime}, y$ and $\eta$ ) and $L_{2}\left(z^{\prime}, x^{\prime}, \xi, y, \eta\right)$ satisfies the estimates of symbols of order -1 w.r.t. the same variables. Proceeding as in the proof of Theorem 2.22 or the proof of Theorem 3.4 (integrations by parts and Taylor expansions of some exponential terms), we find that

$$
f_{\left(z^{\prime \prime}, z^{\prime}, z\right), b, 0}\left(x^{\prime}, \eta\right)=f_{\left(z^{\prime \prime}, z^{\prime}, z\right), c, 0}\left(x^{\prime}, \eta\right)+\max \left(\Delta, \Delta^{\prime}\right) f_{\left(z^{\prime \prime}, z^{\prime}, z\right), 3,0}\left(x^{\prime}, \eta\right),
$$

with

$$
\begin{aligned}
f_{\left(z^{\prime \prime}, z^{\prime}, z\right), c, 0}\left(x^{\prime}, \eta\right)= & \iint e^{i\left\langle x^{\prime}-y \mid \xi-\eta\right\rangle} \chi_{0}(\eta, \xi) L\left(z^{\prime}, x^{\prime}, \xi\right) R\left(z^{\prime}, x^{\prime}, \xi\right) \\
& \times\left(\left\langle y-x^{\prime} \mid L_{1}\left(z^{\prime}, x^{\prime}, \xi, y, \eta\right)\right\rangle+\left\langle\eta-\xi \mid L_{2}\left(z^{\prime}, x^{\prime}, \xi, y, \eta\right)\right\rangle\right) R(z, y, \eta) d y d \xi \\
& =\iint e^{i\left\langle x^{\prime}-y \mid \xi-\eta\right\rangle} \chi_{0}(\eta, \xi)\left(L\left(z^{\prime}, y, \eta\right)-L\left(z^{\prime}, x^{\prime}, \xi\right)\right) R(z, y, \eta) d y d \xi .
\end{aligned}
$$

The operator $\mathcal{F}_{\left(z^{\prime \prime}, z^{\prime}, z\right), 3,0}$, associated to $f_{\left(z^{\prime \prime}, z^{\prime}, z\right), 3,0}\left(x^{\prime}, \eta\right)$, satisfies

$$
\left\|\mathcal{F}_{\left(z^{\prime \prime}, z^{\prime}, z\right), 3,0}\right\|_{\left(\left(H^{(s)}\right)^{k},\left(H^{(s)}\right)^{k}\right)} \leq C,
$$

uniformly w.r.t. $z^{\prime \prime}, z^{\prime}$ and $z$. In fact, to find such an estimate, because of the multiple symbols involved, we use Proposition 4.5 in place of Theorem 1.12 of Section 1.

Setting $f_{\left(z^{\prime \prime}, z^{\prime}, z\right), c}\left(x^{\prime}, \eta\right)=f_{\left(z^{\prime \prime}, z^{\prime}, z\right), c, 0}\left(x^{\prime}, \eta\right)+f_{\left(z^{\prime \prime}, z^{\prime}, z\right), c, \infty}\left(x^{\prime}, \eta\right)$ we obtain

$$
\begin{aligned}
f_{\left(z^{\prime \prime}, z^{\prime}, z\right), c}\left(x^{\prime}, \eta\right)=\iint e^{i\left\langle x^{\prime}-y \mid \xi-\eta\right\rangle}\left(L\left(z^{\prime}, y, \eta\right)-L\left(z^{\prime}, x^{\prime}, \xi\right)\right) R(z, y, \eta) d y d \xi & \\
& =I_{k}-\left(L\left(z^{\prime}, .\right) \# R\left(z^{\prime}, .\right)\right)\left(x^{\prime}, \eta\right)+\Delta f_{\left(z^{\prime \prime}, z^{\prime}, z\right), 4}\left(x^{\prime}, \eta\right),
\end{aligned}
$$

with $f_{\left(z^{\prime \prime}, z^{\prime}, z\right), 4}\left(x^{\prime}, \eta\right)$ bounded w.r.t. $z^{\prime \prime}, z^{\prime}$ and $z$ with values in $S^{0}\left(X^{\prime} \times \mathbf{R}^{n}\right)$, using again Assumption 4.2. This concludes the proof.

Proof of Proposition 4.7. We proceed as in the proof of Proposition 4.6 and use the notation introduced there. The counterpart of the term $f_{\left(z^{\prime \prime}, z^{\prime}, z\right), c}\left(x^{\prime}, \eta\right)$ is then

$$
\iint e^{i\left\langle x^{\prime}-y \mid \xi-\eta\right\rangle}\left(L\left(z^{\prime}, y, \eta\right)-L\left(z^{\prime}, x^{\prime}, \xi\right)\right) d y d \xi
$$

which in fact vanishes.

Proof of Proposition 4.8. We write

$$
\mathcal{H}_{\left(z^{\prime}, z\right)}^{l r} \circ \mathcal{M}\left(z, x, D_{x}\right)=\mathcal{H}_{\left(z^{\prime}, z\right)}^{l r}-\mathcal{H}_{\left(z^{\prime}, z\right)}^{l r} \circ L\left(z, x, D_{x}\right) \circ R\left(z, x, D_{x}\right) .
$$

As a particular case of Proposition 4.7, we have

$$
\mathcal{H}_{\left(z^{\prime}, z\right)}^{l r} \circ L\left(z, x, D_{x}\right)=\mathcal{H}_{\left(z^{\prime}, z\right)}^{l}+\Delta \mathcal{K}_{\left(z^{\prime}, z\right), 1},
$$


with $\left\|\mathcal{K}_{\left(z^{\prime}, z\right), 1}\right\|_{\left(\left(H^{(s)}\right)^{k},\left(H^{(s)}\right)^{k}\right)} \leq C$ uniformly w.r.t. $z^{\prime}$ and $z$. Next, as a particular case of Proposition 4.6 we have

$$
\begin{aligned}
\mathcal{H}_{\left(z^{\prime}, z\right)}^{l r}=\mathcal{H}_{\left(z^{\prime}, z\right)}^{l r} \circ \mathcal{H}_{(z, z)}^{l r} & =\mathcal{H}_{\left(z^{\prime}, z\right)}^{l} \circ \mathcal{H}_{(z, z)}^{r}+\Delta \mathcal{K}_{\left(z^{\prime}, z\right), 2}+\mathcal{M}\left(z^{\prime}, x, D_{x}\right) \\
& =\mathcal{H}_{\left(z^{\prime}, z\right)}^{l} \circ R\left(z, x, D_{x}\right)+\Delta \mathcal{K}_{\left(z^{\prime}, z\right), 2}+\mathcal{M}\left(z^{\prime}, x, D_{x}\right),
\end{aligned}
$$

with $\left\|\mathcal{K}_{\left(z^{\prime}, z\right), 2}\right\|_{\left(\left(H^{(s)}\right)^{k},\left(H^{(s)}\right)^{k}\right)} \leq C$ uniformly w.r.t. $z^{\prime}$ and $z$. The result follows.

\section{A.9 Proofs of Lemmata 4.11 and 4.12}

Proof of Proposition 4.11. From Proposition 2.6 and Theorem 2.22, there exist $S>1$ and $M \geq 0$ such that

$$
\begin{aligned}
& \left\|\mathcal{H}_{\left(z^{\prime}, z\right)}^{r}\right\|_{\left(\left(H^{(s)}\right)^{k},\left(H^{(s)}\right)^{k}\right)} \leq S, \quad\left\|\mathcal{H}_{\left(z^{\prime}, z\right)}^{l r}\right\|_{\left(\left(H^{(s)}\right)^{k},\left(H^{(s)}\right)^{k}\right)} \leq S, \\
& \left\|\mathcal{H}_{\left(z^{\prime}, z\right)}^{l}\right\|_{\left(\left(H^{(s)}\right)^{k},\left(H^{(s)}\right)^{k}\right)} \leq S, \quad \text { and, }\left\|\mathcal{H}_{\left(z^{\prime}, z\right)}\right\|_{\left(\left(H^{(s)}\right)^{k},\left(H^{(s)}\right)^{k}\right)} \leq 1+M \Delta,
\end{aligned}
$$

uniformly w.r.t. $z^{\prime}$ and $z, 0 \leq z \leq z^{\prime} \leq Z$, for $\Delta=z^{\prime}-z$ sufficiently small.

We choose $\Delta_{\mathfrak{F}}$ sufficiently small such that $1+M \Delta_{\mathfrak{F}} \leq S$ and to apply the invoked properties. There is no loss of generality in assuming $l=0$.

If we consider the generic term in the sum of order $r$ in the definition of $\mathcal{J}_{\left(z^{(l)}, z^{(0)}\right)}$ we find

$$
\begin{aligned}
& \| \mathcal{H}_{\left(z^{\left(l^{\prime}\right)}, \ldots, z^{\left(m_{r}+1\right)}\right)}^{l} \circ \mathcal{K} \circ \mathcal{H}_{\left(z^{\left(m_{r}-1\right)}, \ldots, z^{\left(m_{r-1}+1\right)}\right)}^{r} \circ \cdots \\
& \cdots \circ \mathcal{H}_{\left(z^{\left(m_{2}-1\right)}, \ldots, z^{\left(m_{1}+1\right)}\right)}^{r} \circ \mathcal{K} \circ \mathcal{H}_{\left(z^{\left(m_{1}-1\right)}, \ldots, z^{(0)}\right)}^{r} \|_{\left(\left(H^{(s)}\right)^{k},\left(H^{(s)}\right)^{k}\right)} \leq S^{r+2} K^{r}\left(1+M \Delta_{\mathfrak{F}}\right)^{l^{\prime}-3 r-2} .
\end{aligned}
$$

The number of terms in the sum of order $r$ is less than $\left(l^{\prime}-1\right)\left(l^{\prime}-3\right) \cdots\left(l^{\prime}-2 r+1\right) / r$ !. In any case, $l^{\prime}$ being even or odd, we can estimate this number of terms from above by $2^{r}\left(\begin{array}{c}\mathrm{E}\left(l^{\prime} / 2\right) \\ r\end{array}\right)$. In fact, the number of terms in the sum is over estimated but this will suffice to our purpose. We obtain

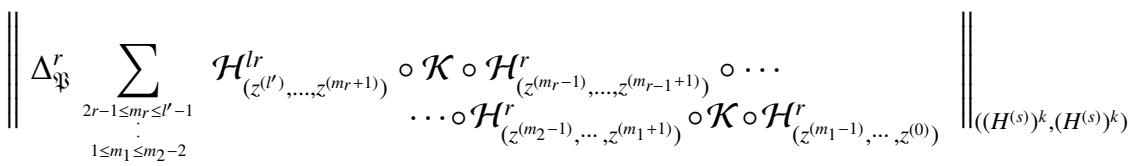

$$
\begin{aligned}
& \leq\left(2 \Delta_{\mathfrak{F}}\right)^{r}\left(\begin{array}{c}
\mathrm{E}\left(l^{\prime} / 2\right) \\
r
\end{array}\right) K^{r} S^{r+2}\left(1+M \Delta_{\mathfrak{F}}\right)^{l^{\prime}-3 r-2} .
\end{aligned}
$$

Observe that this estimation is sharp in the case $r=0$ but becomes rather crude when $r$ becomes large, from the over-estimation made above. In particular, for $r=\mathrm{E}\left(l^{\prime} / 2\right)$ this estimate is much larger than the estimates $\Delta_{\mathfrak{F}}^{\mathrm{E}\left(l^{\prime} / 2\right)} K^{r}$ (in the case $l^{\prime}$ is even) and $\frac{l^{\prime}+1}{2} S \Delta_{\mathfrak{P}}^{\mathrm{E}\left(l^{\prime} / 2\right)} K^{r}$ (in the case $l^{\prime}$ is odd) that we can directly obtain.

Summing the estimates we obtain

$$
\begin{aligned}
& \left\|\mathcal{J}_{\left(z^{\left(l^{\prime}\right)}, z^{(0)}\right)}\right\|_{\left(\left(H^{(s)}\right)^{k},\left(H^{(s)}\right)^{k}\right)} \leq \sum_{r=0}^{\mathrm{E}\left(l^{\prime} / 2\right)}\left(2 \Delta_{\mathfrak{P}}\right)^{r}\left(\begin{array}{c}
\mathrm{E}\left(l^{\prime} / 2\right) \\
r
\end{array}\right) K^{r} S^{r+2}\left(1+M \Delta_{\mathfrak{P}}\right)^{l^{\prime}-3 r-2} \\
& =S^{2}\left(1+M \Delta_{\mathfrak{P}}\right)^{-2} \sum_{r=0}^{\mathrm{E}\left(l^{\prime} / 2\right)}\left(\begin{array}{c}
\mathrm{E}\left(l^{\prime} / 2\right) \\
r
\end{array}\right)\left(\frac{2 \Delta_{\mathfrak{F}} S K}{1+M \Delta_{\mathfrak{F}}}\right)^{r}\left(\left(1+M \Delta_{\mathfrak{F}}\right)^{2}\right)^{l^{\prime} / 2-r} .
\end{aligned}
$$

In the case where $l^{\prime}$ is even we obtain

$$
\left\|\mathcal{J}_{\left(z^{\left(l^{\prime}\right.}, z^{(0)}\right)}\right\|_{\left(\left(H^{(s)}\right)^{k},\left(H^{(s)}\right)^{k}\right)} \leq S^{2}\left(1+M \Delta_{\mathfrak{F}}\right)^{-2}\left(\frac{2 \Delta_{\mathfrak{F}} S K}{1+M \Delta_{\mathfrak{F}}}+\left(1+M \Delta_{\mathfrak{F}}\right)^{2}\right)^{\mathrm{E}\left(l^{\prime} / 2\right)} .
$$

Thus, there exists $C \geq 0$ such that

$$
\left\|\mathcal{J}_{\left(z^{\left(l^{\prime}\right)}, z^{(0)}\right)}\right\|_{\left(\left(H^{(s)}\right)^{k},\left(H^{(s)}\right)^{k}\right)} \leq S^{2}\left(1+C \Delta_{\mathfrak{F}}\right)^{\mathrm{E}\left(l^{\prime} / 2\right)} \leq S^{2}(1+Z C / N)^{N},
$$

which is bounded, with $S^{2} \exp (C Z)$ as an upper bound. The case where $l^{\prime}$ is odd yields a similar bound. 
Proof of Proposition 4.12. Let us first consider the composition of $\mathcal{H}_{\left(z^{\left(l^{\prime}+1\right)}, z^{\left(l^{\prime}\right)}\right)}^{l r}$ with the term of order 0 in $\mathcal{J}_{\left(z^{\left(l^{\prime}\right)}, z^{(l)}\right)}$, i.e. $\mathcal{H}_{\left(z^{\left(l^{\prime}\right)}, \ldots, z^{(l)}\right)}^{l r}$. By proposition 4.7, we have, since $l^{\prime}-l \geq 2$,

$$
\mathcal{H}_{\left(z^{\left(l^{\prime}+1\right)}, z^{\left(l^{\prime}\right)}\right)}^{l r} \circ \mathcal{H}_{\left(z^{\left(l^{\prime}\right)}, \ldots, z^{(l)}\right)}^{l r}=\mathcal{H}_{\left(z^{\left(l^{\prime}+1\right)}, \ldots, z^{(l)}\right)}^{l r}+\Delta_{\mathfrak{P}} \mathcal{K} \circ \mathcal{H}_{\left(z^{\left(l^{\prime}-1\right)}, \ldots, z^{(l)}\right)}^{r} .
$$

Let us now consider the composition of $\mathcal{H}_{\left(z^{\left(l^{\prime}+1\right)}, z^{\left(l^{\prime}\right)}\right)}^{l}$ with the term of order $r \geq 1$ in $\mathcal{J}_{\left(z^{\left(l^{\prime}\right)}, z^{(l)}\right)}$ in (4.8) in Definition 4.9. We address three cases in the sum defining the term of order $r$ : (i) $m_{r}=l^{\prime}-1$, (ii) $m_{r}=l^{\prime}-2$, and (iii) $m_{r} \leq l^{\prime}-3$. Case (iii) does not occur if $l^{\prime}-l \leq 3$. If $l^{\prime}-l$ is even and if we consider the term of order $r=E\left(\left(l^{\prime}-l\right) / 2\right)=\left(l^{\prime}-l\right) / 2$ in (4.8) then only case (i) occurs. If $l^{\prime}-l$ is odd and if we consider the term of order $r=E\left(\left(l^{\prime}-l\right) / 2\right)=\left(l^{\prime}-l-1\right) / 2$ in (4.8) then Case (iii) does not occur.

Case (i): $m_{r}=l^{\prime}-1$. The term under consideration in the sum of order $r$ in (4.8) is

$$
\Delta_{\mathfrak{P}}^{r} \sum_{\substack{l+2 r-3 \leq m_{r-1} \leq l^{\prime}-3 \\ \vdots \\ l+3 \leq m_{2} \leq m_{3}-2 \\ l+1 \leq m_{1} \leq m_{2}-2}} \mathcal{K} \circ \mathcal{H}_{\left(z^{\left(l^{\prime}-2\right)}, \ldots, z^{\left(m_{r-1}+1\right)}\right)}^{r} \circ \cdots \circ \mathcal{H}_{\left(z^{\left(m_{2}-1\right)}, \cdots, z^{\left(m_{1}+1\right)}\right)}^{r} \circ \mathcal{K} \circ \mathcal{H}_{\left(z^{\left(m_{1}-1\right)}, \cdots, z^{(l)}\right)^{r}}^{r} .
$$

After composition with $\mathcal{H}_{\left(z^{\left(l^{\prime}+1\right)}, z^{\left.l^{\prime}\right)}\right)}^{l}$ from the 1.h.s., we simply obtain

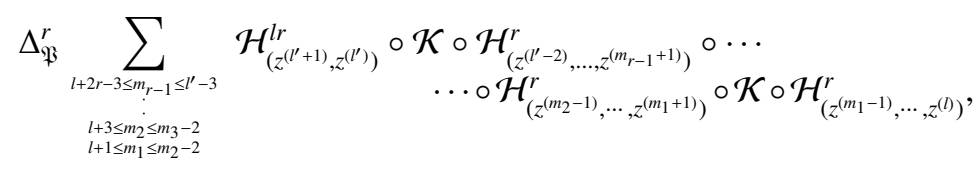

which is of order $r$.

Case (ii): $m_{r}=l^{\prime}-2$. The term under consideration in the sum of order $r$ in (4.8) is

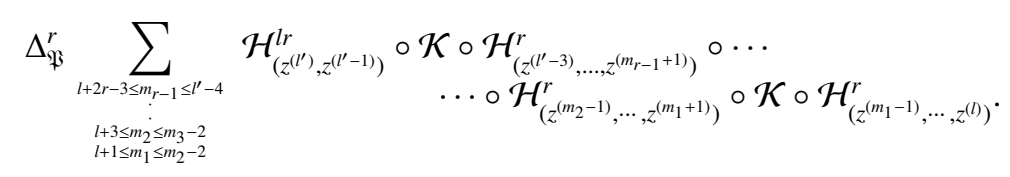

By Proposition 4.6, we have

$$
\mathcal{H}_{\left(z^{\left(l^{\prime}+1\right)}, z^{\left(l^{\prime}\right)}\right)}^{l r} \circ \mathcal{H}_{\left(z^{\left(l^{\prime}\right)}, z^{\left(l^{\prime}-1\right)}\right)}^{l r}=\mathcal{H}_{\left(z^{\left(l^{\prime}+1\right)}, \ldots, z^{\left(l^{\prime}-1\right)}\right)}^{l r}+\Delta_{\mathfrak{P}} \mathcal{K}+\mathcal{M}_{z^{\left(l^{\prime}+1\right)}} .
$$

We thus obtain three terms when composing with $\mathcal{H}_{\left(z^{\left(l^{\prime}+1\right)}, z^{\left(l^{\prime}\right)}\right)}^{l r}$ from the 1.h.s.:

$$
\Delta_{\mathfrak{F}}^{r} \sum_{\substack{l+2 r-3 \leq m_{r-1} \leq l^{\prime}-4 \\ \cdots \\ l+3 \leq m_{2} \leq m_{3}-2 \\ l+1 \leq m_{1} \leq m_{2}-2}} \mathcal{H}_{\left(z^{\left(l^{\prime}+1\right)}, \ldots, z^{\left(l^{\prime}-1\right)}\right)}^{l r} \circ \mathcal{K} \circ \mathcal{H}_{\left(z^{\left(l^{\prime}-3\right)}, \ldots, z^{\left(m_{r-1}+1\right)}\right)}^{r} \circ \cdots \mathcal{H}_{\left(z^{\left(m_{2}-1\right)}, \cdots, z^{\left(m_{1}+1\right)}\right)}^{r} \circ \mathcal{H}_{\left(z^{\left(m_{1}-1\right)}, \cdots, z^{(l)}\right)}^{r},
$$

and

$$
\Delta_{\mathfrak{P}}^{r} \mathcal{M}_{z^{\left(l^{\prime}+1\right)}} \circ \mathcal{K} \circ \sum_{\substack{l+2 r-3 \leq m_{r-1} \leq l^{\prime}-4 \\ \vdots \\ l+3 \leq m_{2} \leq m_{3}-2 \\ l+1 \leq m_{1} \leq m_{2}-2}} \mathcal{H}_{\left(z^{\left(l^{\prime}-3\right)}, \ldots, z^{\left(m_{r-1}+1\right)}\right)}^{r} \circ \mathcal{K}^{\cdots} \circ \mathcal{H}_{\left(z^{\left(m_{2}-1\right)}, \cdots, z^{\left(m_{1}+1\right)}\right)}^{r} \circ \mathcal{K}^{r\left(m_{r-1}-1\right)} \circ \mathcal{H}_{\left(z^{\left(m_{1}-1\right)}, \cdots, z^{(l)}\right)}^{r},
$$

which are of order $r$ and

$$
\Delta_{\mathfrak{P}}^{r+1} \sum_{\substack{l+2 r-3 \leq m_{r-1} \leq l^{\prime}-4 \\ ! \\ l+3 \leq m_{2} \leq m_{3}-2 \\ l+1 \leq m_{1} \leq m_{2}-2}} \mathcal{K} \circ \mathcal{K} \circ \mathcal{H}_{\left(z^{\left(l^{\prime}-3\right)}, \ldots, z^{\left(m_{r-1}+1\right)}\right)}^{r} \circ \mathcal{K}_{\left(z^{\left(m_{r-1}-1\right)}, \ldots, z^{\left(m_{r-2}+1\right)}\right)}^{r} \circ \cdots
$$

which is of order $r+1$. 
Case (iii): $m_{r} \leq l^{\prime}-3$. The term under consideration in the sum of order $r$ in (4.8) is

$$
\Delta_{\mathfrak{P}}^{r} \sum_{\substack{l+2 r-1 \leq m_{r} \leq l^{\prime}-3 \\ \vdots \\ l+3 \leq m_{2} \leq m_{3}-2 \\ l+1 \leq m_{1} \leq m_{2}-2}} \mathcal{H}_{\left(z^{\left(l^{\prime}\right)}, z^{\left(l^{\prime}-1\right)}\right)}^{l} \circ \mathcal{H}_{\left(z^{\left(l^{\prime}-1\right)}, \ldots, z^{\left(m_{r}+1\right)}\right)}^{r} \circ \mathcal{K}^{r} \circ \mathcal{H}_{\left(z^{\left(m_{r}-1\right)}, \ldots, z^{\left(m_{r-1}+1\right)}\right)}^{r} \circ \cdots
$$

which, in fact, contains all the remaining terms of order $r$ in $\mathcal{J}_{\left(z^{\left(l^{\prime}\right)} z^{(l)}\right)}$. By Proposition 4.7 we have

$$
\mathcal{H}_{\left(z^{\left(l^{\prime}+1\right)}, z^{\left(l^{\prime}\right)}\right)}^{l r} \circ \mathcal{H}_{\left(z^{\left(l^{\prime}\right)}, z^{\left(l^{\prime}-1\right)}\right)}^{l}=\mathcal{H}_{\left(z^{\left(l^{\prime}+1\right)}, \ldots, z^{\left(l^{\prime}-1\right)}\right)}^{l}+\Delta_{\mathfrak{P}} \mathcal{K}
$$

After composition with $\mathcal{H}_{\left(z^{\left(l^{\prime}+1\right)}, z^{\left(l^{\prime}\right)}\right)}^{l r}$ from the 1.h.s. we thus obtain two terms:

which is of order $r$ and finally

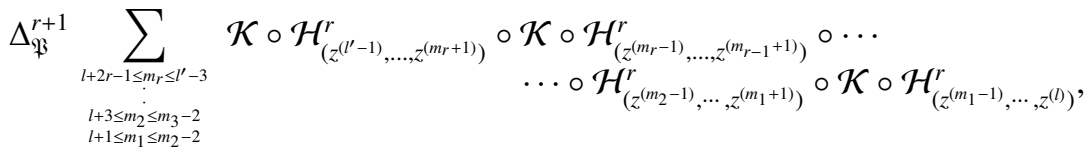

which is of order $r+1$.

Let us first consider the resulting terms in which the operator $M_{z^{\left(l^{\prime}+1\right)}}$ does not occur.

Term of order 0 There is only one term of order 0 coming from (A.25), i.e. $\mathcal{H}_{\left(z^{\left(l^{\prime}+1\right)}, \ldots, z^{(l)}\right)}^{l r}$.

Terms of order $\mathbf{1} \leq \boldsymbol{r} \leq \mathbf{E}\left(\left(\boldsymbol{l}^{\prime}+\mathbf{1}-\boldsymbol{l}\right) / \mathbf{2}\right)-2$ The terms originate either from terms of order $r$ in $\mathcal{J}_{\left(z^{\left(l^{\prime}\right)}, z^{(l)}\right)}$ and can be found in (A.26), (A.27) and (A.30), or they originate from terms of order $r-1$ in $\mathcal{J}_{\left(z^{\left(l^{\prime}\right)}, z^{(l)}\right)}$ and can be found in (A.29) and (A.31) (and (A.25) in the case $r=1$ ). Gathering these terms we obtain

$$
\Delta_{\mathfrak{P}}^{r} \sum_{\substack{l+2 r-1 \leq m_{r} \leq l^{\prime} \\ l+3 \leq m_{2} m_{3}-2 \\ l+1 \leq m_{1} \leq m_{2}-2}} \mathcal{H}_{\left(z^{\left(l^{\prime}+1\right)}, \ldots, z^{\left(m_{r}+1\right)}\right)}^{l r} \circ \mathcal{K} \circ \mathcal{H}_{\left(z^{\left(m_{r}-1\right)}, \ldots, z^{\left(m_{r-1}+1\right)}\right)}^{r} \circ \cdots \mathcal{H}_{\left(z^{\left(m_{2}-1\right)}, \cdots, z^{\left(m_{1}+1\right)}\right)}^{r} \circ \mathcal{K} \circ \mathcal{H}_{\left(z^{\left(m_{1}-1\right)}, \cdots, z^{(l)}\right)}^{r}
$$

which is exactly the term of order $r$ in $\mathcal{J}_{\left(z^{\left(l^{\prime}+1\right)}, z^{(l)}\right)}$.

Terms of order $\mathbf{E}\left(\left(l^{\prime}+\mathbf{1}-l\right) / \mathbf{2}\right)-\mathbf{1}$ The case where $l^{\prime}-l$ is even is treated as in the previous point. We now consider the case $l^{\prime}-l$ odd. The terms originate either from terms of order $r=\mathrm{E}\left(\left(l^{\prime}+1-l\right) / 2\right)-1=$ $\left(l^{\prime}-l-1\right) / 2$ in $\mathcal{J}_{\left(z^{\left(l^{\prime}\right)}, z^{(l)}\right)}$ and can be found in (A.26), (A.27), since Case (iii) does not occur here, or they originate from terms of order $r-1$ in $\mathcal{J}_{\left(z^{\left(l^{l}\right)}, z^{(l)}\right)}$ and can be found in (A.29) and (A.31). Gathering these terms we obtain the term of order $r$ in $\mathcal{J}_{\left(z^{\left(l^{\prime}+1\right)}, z^{(l)}\right)}$.

Terms of order $\mathbf{E}\left(\left(l^{\prime}+\mathbf{1}-l\right) / 2\right)$ In the case where $l^{\prime}-l$ is even, then $r=\mathrm{E}\left(\left(l^{\prime}+1-l\right) / 2\right)=\left(l^{\prime}-l\right) / 2$. The terms originate either from terms of order $r$ in $\mathcal{J}_{\left(z^{\left(l^{\prime}\right)}, z^{(l)}\right)}$ and can be found in (A.26) as only case (i) occurs here, or they originate from terms of order $r-1$ in $\mathcal{J}_{\left(z^{\left(l^{\prime}\right)} z^{(l)}\right)}$ and can be found in (A.29) and (A.31). Gathering these terms we obtain the term of order $r=\left(l^{\prime}-l\right) / 2$ in $\mathcal{J}_{\left(z^{\left(l^{\prime}+1\right)}, z^{(l)}\right)}$.

In the case where $l^{\prime}-l$ is odd, the terms of order $\mathrm{E}\left(\left(l^{\prime}-l\right) / 2\right)=\left(l^{\prime}-1-l\right) / 2$ in $\mathcal{J}_{\left(z^{\left(l^{\prime}\right)} z^{(l)}\right)}$ are (case (iii) does not occur)

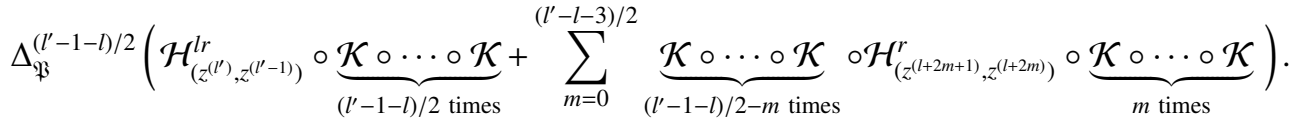

After composition with $\mathcal{H}_{\left(z^{\left(l^{\prime}+1\right)}, z^{\left(l^{\prime}\right)}\right)}^{l r}$ from the 1.h.s. the only term of order $\mathrm{E}\left(\left(l^{\prime}+1-l\right) / 2\right)=\left(l^{\prime}+1-l\right) / 2$ is given by (A.29), viz., $\Delta_{\mathfrak{P}}^{\left(l^{\prime}+1-l\right) / 2} \underbrace{\mathcal{K} \circ \cdots \circ \mathcal{K}}_{\left(l^{\prime}+1-l\right) / 2 \text { times }}$, which is exactly the term of order $\left(l^{\prime}+1-l\right) / 2$ in $\mathcal{J}_{\left(z^{\left(l^{\prime}+1\right)}, z^{(l)}\right)}$. 
We now consider the terms in which the operator $\mathcal{M}_{z^{\left(l^{\prime}+1\right)}}$ occur. Observe that there is no term of order 0 . The term of order $r>0$, given in (A.28) is in fact the term of order $r-1$ in the definition of $\tilde{\mathcal{J}}_{\left(z^{\left(l^{\prime}-3\right)}, z^{(l)}\right)}$ composed from the 1.h.s. by $\Delta_{\mathfrak{P}} \mathcal{M}_{z^{\left(l^{\prime}+1\right)}} \circ \mathcal{K}$.

In the case where $l^{\prime}-l$ is even, then the highest-order term with the operator $\mathcal{M}_{z^{\left(l^{\prime}+1\right)}}$ occurring originates from the term of order $\left(l^{\prime}-l\right) / 2-1$ in $\mathcal{J}_{\left(z^{\left(l^{\prime}\right)}, z^{(l)}\right)}$ and yields the last term in $\tilde{\mathcal{J}}_{\left(z^{\left(l^{\prime}-3\right)}, z^{(l)}\right)}$, which is of order $\left(l^{\prime}-l\right) / 2-2=\mathrm{E}\left(\left(l^{\prime}-3-l\right) / 2\right)$, composed from the 1.h.s. by $\Delta_{\mathfrak{P}} \mathcal{M}_{z^{\left(l^{\prime}+1\right)}} \circ \mathcal{K}($ see $(\mathrm{A} .28))$. In the case where $l^{\prime}-l$ is odd, then the highest-order term with an occurrence of the operator $\mathcal{M}_{z^{\left(l^{\prime}+1\right)}}$ originates from the term of order $\mathrm{E}\left(\left(l^{\prime}-l\right) / 2\right)=\left(l^{\prime}-1-l\right) / 2$ in $\mathcal{J}_{\left(z^{\left(l^{\prime}\right)}, z^{(l)}\right)}$ and is given in (A.28), i.e.

$$
\Delta_{\mathfrak{P}}^{\left(l^{\prime}-1-l\right) / 2} \mathcal{M}_{z^{\left(l^{\prime}+1\right)}} \circ \mathcal{K} \circ \underbrace{\mathcal{K} \circ \cdots \circ \mathcal{K}}_{\left(l^{\prime}-3-l\right) / 2 \text { times }}
$$

which is the term of order $\left(l^{\prime}-3-l\right) / 2$ in the definition of $\tilde{\mathcal{J}}_{\left(z^{\left(l^{\prime}-3\right)}, z^{(l)}\right)}$, composed from the 1.h.s. by $\Delta_{\mathfrak{P}} \mathcal{M}_{z^{\left(l^{\prime}+1\right)}} \circ$ $\mathcal{K}$.

Adapting the proof of Lemma 4.11, we obtain the norm estimate (4.10) for operators of the form of $\tilde{\mathcal{J}}_{\left(z^{\left(l^{\prime}\right)}, z^{(l)}\right)}$.

Acknowledgement: The author wishes to thank Olivier Guès for many helpful discussions on hyperbolic systems and Didier Robert for early discussions on the content ot the article. The author also wishes to thank Hiroshi Isozaki for inspiring discussions that helped in the correction of a flaw in one of the proofs.

\section{References}

[1] K. Asada and D. Fujiwara. On some oscillatory integral transformations in $L^{2}\left(\mathbf{R}^{n}\right)$. Japan. J. Math. (N.S.), 4:299-361, 1978.

[2] J.-M. Bony. Sur l'ingalit de Fefferman-Phong. Smin. qu. Driv. Partielles, cole Polytech., Palaiseau, 1999.

[3] A. Boulkhemair. Estimations $L^{2}$ précisées pour des intégrales oscillantes. Comm. Partial Differential Equations, 22:165-184, 1997.

[4] J. Chazarain and A. Piriou. Introduction to the Theory of Linear Partial Differential Equations. NorthHolland, Amsterdam, 1982.

[5] M. V. de Hoop. Microlocal analysis of seismic inverse scattering. In G. Uhlmann, editor, Inside Out, Inverse Problems and Applications, Cambridge, 2004. Cambridge University Press.

[6] M. V. de Hoop and A. T. de Hoop. Elastic wave up/down decomposition in inhomogeneous and anisotropic media: an operator approach and its approximations. Wave Motion, 20:57-82, 1994.

[7] M. V. de Hoop, J. Le Rousseau, and B. Biondi. Symplectic structure of wave-equation imaging: A path-integral approach based on the double-square-root equation. Geoph. J. Int., 153:52-74, 2003.

[8] M. V. de Hoop, J. Le Rousseau, and R.-S. Wu. Generalization of the phase-screen approximation for the scattering of acoustic waves. Wave Motion, 31:43-70, 2000.

[9] J. J. Duistermaat. Fourier Integral Operators. Birkhäuser, Boston, 1996.

[10] K.-J. Engel and R. Nagel. One-Parameter Semigroups for Linear Evolution Equations. SpringerVerlag, Berlin, 1999.

[11] D. Fujiwara and N. Kumano-go. Smooth functional derivatives in feynman path integrals by time slicing approximation. Bull. Sci. Math., 129:57-79, 2005.

[12] L. Hörmander. Fourier integral operators I. Acta Math., 127:79-183, 1971.

[13] L. Hörmander. $L^{2}$ estimate for Fourier integral operators with complex phase. Ark. Mat., 21(2):283$307,1983$. 
[14] L. Hörmander. The Analysis of Linear Partial Differential Operators, volume IV. Springer-Verlag, 1985.

[15] L. Hörmander. The Analysis of Linear Partial Differential Operators, volume III. Springer-Verlag, 1985. Second printing 1994.

[16] L. Hörmander. The Analysis of Linear Partial Differential Operators, volume I. Springer-Verlag, second edition, 1990.

[17] T. Ichinose and H. Tamura. Note on the norm convergence of the unitary trotter product formula. Lett. Math. Phys., 70:65-81, 2004.

[18] T. Kato. Linear evolution equations of "hyperbolic" type. J. Fac. Sci. Univ. Tokyo, Sec. I, 17:241-258, 1970.

[19] T. Kato. Perturbation Theory for Linear Operators. Springer-Verlag, Berlin, 1980.

[20] H. Kitada and H. Kumano-go. A family of Fourier integral operators and the fundamental solultion for a Schrödinger equation. Osaka J. Math., 18:291-360, 1981.

[21] H. Kumano-go. Algebras of pseudo-differential operators. J. Fac. Sci. Univ. Tokyo, 7:31-50, 1970.

[22] H. Kumano-go. A calculus of Fourier integral operators on $\mathbf{R}^{N}$ and the fundamental solution for an operator of hyperbolic type. Comm. Partial Differential Equations, 1(1):1-44, 1976.

[23] H. Kumano-go. Pseudo-Differential Operators. MIT Press, Cambridge, 1981.

[24] H. Kumano-go and K. Taniguchi. Fourier integral operators of multi-phase and the fundamental solution for a hyperbolic system. Funkcialaj Ekvacioj, 22:161-196, 1979.

[25] H. Kumano-go, K. Taniguchi, and Y. Tozaki. Multi-products of phase functions for Fourier integral operators with an application. Comm. Partial Differential Equations, 3(4):349-380, 1978.

[26] N. Kumano-go. A construction of the fundamental solution for Schrödinger equations. J. Math. Sci. Univ. Tokyo, 2:441-498, 1995.

[27] N. Kumano-go. Feynman path integrals as analysis on path space by time slicing approximation. Bull. Sci. Math., 128:197-251, 2004.

[28] P. D. Lax and L. Nirenberg. On stability for difference schemes: A sharp form of Gårding's inequality. Comm. Pure Appl. Math., 19:473-492, 1966.

[29] J. Le Rousseau. Fourier-integral-operator approximation of solutions to pseudodifferential firstorder hyperbolic equations I: convergence in Sobolev spaces. Comm. Partial Differential Equations, 31:867-906, 2006.

[30] J. Le Rousseau. On the convergence of some products of Fourier integral operators. Asymptotic Anal., 51:189-207, 2007.

[31] J. Le Rousseau and M. V. de Hoop. Generalized-screen approximation and algorithm for the scattering of elastic waves. Q. J. Mech. Appl. Math., 56:1-33, 2003.

[32] J. Le Rousseau and G. Hörmann. Fourier-integral-operator approximation of solutions to pseudodifferential first-order hyperbolic equations II: microlocal analysis. J. Math. Pures Appl., 86:403-426, 2006.

[33] G. Métivier and K. Zumbrun. Hyperbolic boundary value problems for symmetric systems with variable mutiplicities. J. Differential Equations, 211:61-134, 2005.

[34] A. Pazy. Semigroups of Linear Operators and Applications to Partial Differential Equations. SpringerVerlag, New York, 1983. 
[35] M. Ruzhansky and M. Sugimoto. Global $L^{2}$-boundedness theorems for a class of Fourier integral operators. Comm. Partial Differential Equations, 31:547-569, 2006.

[36] C. C. Stolk. A pseudodifferential equation with damping for one-way wave propagation in inhomogeneous media. Wave Motion, 40(2):111-121, 2004.

[37] C. C. Stolk. Parametrix for a hyperbolic intial value problem with dissipation in some region. Asymptotic Anal., 43(1-2):151-169, 2005.

[38] C. C. Stolk and M. V. de Hoop. Microlocal analysis of seismic inverse scattering in anisotropic, elastic media. Comm. Pure Appl. Math., 55:261-301, 2002.

[39] M. E. Taylor. Pseudodifferential Operators. Princeton University Press, Princeton, New Jersey, 1981.

[40] R. Vaillancourt. A simple proof of Lax-Nirenberg Theorems. Comm. Pure Appl. Math., 23:151-163, 1970. 